\title{
Quantitation Strategies in Optically Sectioning Fluorescence Microscopy
}

\section{PhD Thesis}

in partial fulfilment of the requirements

for the degree "Doctor of Philosophy $(\mathrm{PhD}) /$ Dr. rer. nat"

in the Neurosciences Program

at the Georg August University Göttingen,

Faculty of Biology

submitted by

\section{Arwed Weigel}

born in

\section{Kassel, Germany}


\%o my parents 


\section{Declaration}

Herewith I declare that I prepared the $\mathrm{PhD}$ Thesis

"Quantitation Strategies in Optically Sectioning Fluorescence Microscopy"

on my own and with no other sources and aids than quoted.

Göttingen, December $8^{\text {th }}, 2008$ 


\section{Contents}

Dedication ...............................

Declaration ....................

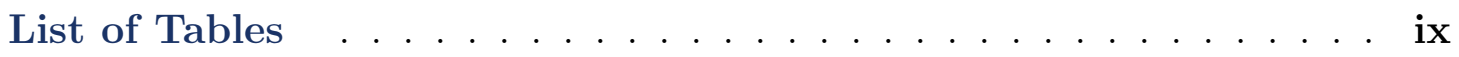

List of Figures . . . . . . . . . . . . . . . . . xi

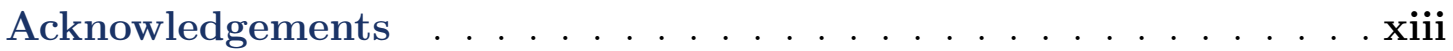

I Introduction . . . . . . . . . . . . . . . . . . 1

II Quantitation in Optically Sectioning Microscopes . . . . . . . 7

2.1 Concepts of Resolution and Optical Sectioning . . . . . . . . . . 7

2.1.1 Definitions for Lateral and Axial Resolution . . . . . . . . . 8

2.1.2 Types of Optically Sectioning Microscopes . . . . . . . . . 11

2.2 Theory and Applications of eSIPcharts . . . . . . . . . . . . 14

2.2.1 Calibration Standards . . . . . . . . . . . . . . . . . 14

2.2.2 Modelling the Axial Intensity Response . . . . . . . . . . 16

2.2.3 Conversion of ADUs to Photon Numbers . . . . . . . . . . 21

2.2.4 Imaging Parameters in eSIPcharts . . . . . . . . . . . . 23

2.3 Extended SIPchart analysis . . . . . . . . . . . . . . . . . . 32

2.4 Discussion . . . . . . . . . . . . . . . . . . . . . . . 38

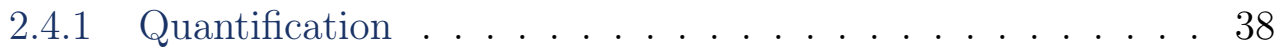

2.4.2 Fluorescent Solution as a Calibration Standard . . . . . . . 39

2.4.3 eSIPcharts and Complementary Calibration . . . . . . . . . 41

2.5 Exemplary eSIPcharts of Some Standard Sectioning Microscopes . 43

III Quantitation in Structured Illumination Microscopy . . . . . . . 49

3.1 Principles of Linear Grid Structured Illumination . . . . . . . . . . 49

3.1.1 Confocal vs. Widefield Epifluorescence Microscopy . . . . . 49

3.1.2 Structured Illumination Microscopy . . . . . . . . . . . . 51

3.1.3 The ApoTome Setup . . . . . . . . . . . . . . . . 55

3.2 Characterisation of the ApoTome . . . . . . . . . . 56 
3.2.1 Resolution .................... 56

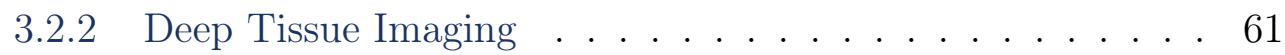

3.2 .3 Anisotropy ....................... 64

3.2.4 Spectral Versatility . . . . . . . . . . . . . . . 69

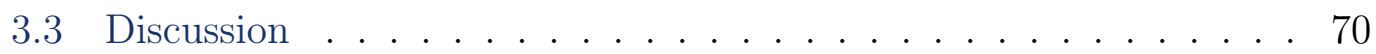

3.4 Appendix: Artefacts in Grid-SIM . . . . . . . . . . . . . 72

IV Summary . . . . . . . . . . . . . . . . . . . 75

V Zusammenfassung . . . . . . . . . . . . . 77

Bibliography ....................... 81

Curriculum Vitae . . . . . . . . . . . . . . . . . . 93

List of Publications . . . . . . . . . . . . . . . . . . 95 


\section{List of Tables}

1 Comparison of PSF and eSIPchart . . . . . . . . . . . . 42

2 Normalised Grid Frequencies in the ApoTome . . . . . . . . . . . 57 


\section{List of Figures}

Fluorescence Transitions . . . . . . . . . . . . . . . . . . 2

Definitions of Resolution . . . . . . . . . . . . . . . 8

First Confocal Microscope . . . . . . . . . . . . . . . . . 10

First Patent on a 'Confocal' Microscope . . . . . . . . . . . . . . 11

Convolution of the Microscope's PSF With Extended Objects. . . 15

Effect of the Skew Parameter on the Fit Function . . . . . . . . . 17 Comparison of Three Fit Functions For Axial Profile Fitting . . . 18 Comparison of the Residuals of Three Fit Function . . . . . . . . 19 Photon Amplification Over Wavelength . . . . . . . . . . . . 22 eSIPchart: Intensity . . . . . . . . . . . . . . . 24 eSIPchart: Resolution . . . . . . . . . . . . . 25 eSIPchart: Position . . . . . . . . . . . . . . 26

Orientation of the Fluorescent Layer in the Image Stack . . . . . 27 eSIPchart: Offset .................... . . 28 eSIPchart: Skew . . . . . . . . . . . . . . . . 29

eSIPchart: Decay Parameter . . . . . . . . . . . . . 31

eSIPchart: Weight Factor . . . . . . . . . . . . . . . . . 32

eSIPchart: Detector Gain . . . . . . . . . . . . . . 33

eSIPchart: Exemplary Data Traces. . . . . . . . . . . . . 34

eSIPchart for the LSM 510 META . . . . . . . . . . . . 35

Chromatic Aberration in Multichannel Systems: Intensity . . . . 36

Chromatic Aberration in Multichannel Systems: Resolution . . . 37

Chromatic Aberration in Multichannel Systems: Focal Plane . . . 38 eSIPchart Olympus Fluoview 1000/layer . . . . . . . . . . . . 44 eSIPchart Zeiss LSM710/layer . . . . . . . . . . . . . . . . . 45 eSIPchart Andor Revolution Spinning Disc . . . . . . . . . . . . . 46 eSIPchart Leica TCS/SP2 . . . . . . . . . . . . . . . . . 47 
eSIPchart LSM510/solution . . . . . . . . . . . . . . . . . . . 48

$31 \quad$ SIM Principle in the ApoTome . . . . . . . . . . . . . . 52

32 Dependence of Sectioning Strength on $\tilde{\nu} \ldots \ldots$. . . . . . . 54

33 Scheme of the ApoTome Beam Path . . . . . . . . . . . . 56

$34 \quad$ PSFs in SIM . . . . . . . . . . . . . . . 57

35 Resolution as a Function of the Pinhole Diameter . . . . . . . . . 59

36 Field of Axial Resolution _................. 60

37 Deep Tissue Imaging with the ApoTome . . . . . . . . . . . . . 63

38 Anisotropy of Resolution in the ApoTome . . . . . . . . . . . 66

39 Effective Object Transfer Function in Grid SIM . . . . . . . . . . 67

40 Effective Object Transfer Function in SIM in Focus . . . . . . . . 68

41 Multicolour Staining in the ApoTome . . . . . . . . . . . . 70 


\section{Acknowledgements}

I wish to express my sincere gratefulness to:

My supervisor Dr. André Zeug, without whom this thesis would not have been possible. André was the first person I could ask whenever I faced problems during my work, and he was the one who would join me in facing these challenges to solve them in every minute detail. Thanks for your $24 / 7$ support, and for becoming a friend in the past years.

Prof. Dr. Dr. Schild, the head of the Department for Neurophysiology and Cellular Biophysics, for giving me the opportunity to do my thesis in his department where I could exploit all the resources to fuel my ideas, for many fruitful discussions that did not always need to be related to actual scientific questions, and for sharing his ideas with me.

Prof. Dr. Walter Stühmer for kindly accepting to be the second referee for this thesis.

Stephan Junek for frequently sharing his Matlab expertise with me, for writing the indispensable matVis-software, for help in live staining experiments, and for all the critical discussions that represent the essence of science.

Mihai Alevra for Matlab support and proofreading.

Gudrun Federkeil for the preparation of cryosections and cell cultures, Joško Kuduz for support in immunohistochemistry experiments, and Eugen Kludt for preparing as many electroporated tadpoles as required.

The mechanical and electronic workshop for the realisation of uncountable ideas.

Juriaan Zwier for providing me with my "holy" thin homogeneous fluorescent layer, which was my "precious", and of which I was seriously envied.

All the members of the Department of Neurophysiology and Cellular Biophysics for all the small discussions that help preparing a thesis, for a constant coffee supply and a nice working atmosphere.

My friends in Göttingen who made my time here a valuable part of my life. 
My friends all over the world, especially Andrea, Konrad, and Netaya, for practical and mental support in every area of my life.

My parents, siblings, grandmother, sister-in-law, niece, nephew, godparents, father and mother-in-law, whose unquestioning support means no less than the foundation of my being. Ohne Euch wäre ich nicht der, der ich bin. Danke für alles!

Gesa for always and forever being with me, for bearing me in these stressful times, and for making me the happiest person on earth. Danke! 


\section{Introduction}

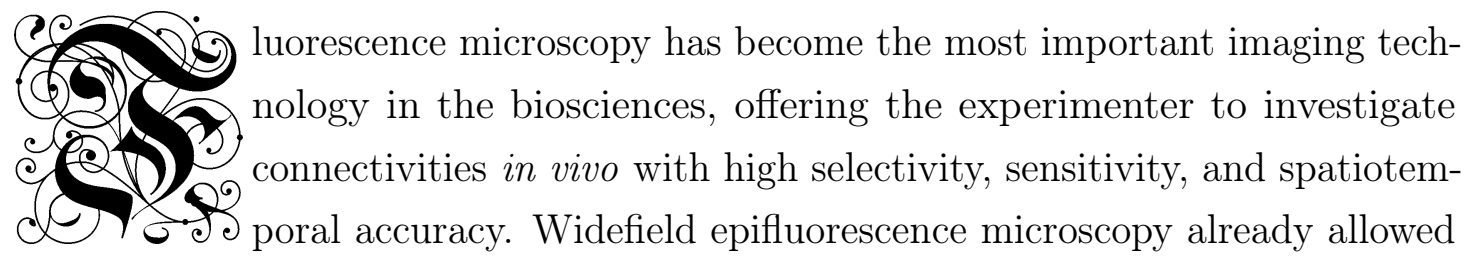

the observation of single molecules in living cells, a sensitivity that is unchallenged by all brightfield techniques. With the advent of commercial optically sectioning fluorescence microscopes in the middle-eighties a boom in the biosciences can be observed that did not decelerate until now. The new technology made it possible to address questions that remained unanswered for decades. Optically sectioning fluorescence microscopy is an extremely dynamic field in science, and new imaging strategies which optimise the extraction of certain parameters still revolutionise our knowledge about imaging on a yearly basis. One has to ask: why is it fluorescence microscopy that leads to these rapid advances in a scientific area which seemed to be completed for more than 100 years after E. ABBE's essay about microscopy (Abbe, 1873)?

The principle of fluorescence allows to investigate a probe without background. When a signal is detected, one can be sure that it is a fluorescence photon. This results in a contrast of $100 \%$ which fluorescence microscopes inherently have. In order to understand which feature of fluorescence is responsible for this ultrahigh sensitivity, I will briefly outline the basic principles of fluorescence.

A typical fluorophore is an aromatic molecule with an extended $\pi$ bond system. The absorption of one photon of an energy $E=h \nu$ high enough to bridge the fluorophore's electronic energy gap leads to a transition into the excited state according to the FRANCK-CONDON-principle: the transition is so fast that the nuclear coordinates are not changed. The fluorophore will get rid of its excess vibrational energy within picoseconds by internal conversion and stay on the first electronically excited vibrationally relaxed state for the duration of the fluorescence lifetime, which is typically in the order of nanoseconds. The fluorophore falls back into a vibrationally excited ground state and loses the vibrational energy by internal conversion upon the emission of a photon. For simplicity, I will omit the discussion of higher electronic states and the triplet state here.

Naturally, the energy of the excitation photon is higher than the energy of the emitted photon, the energy being lost in the internal conversion. This wavelength 


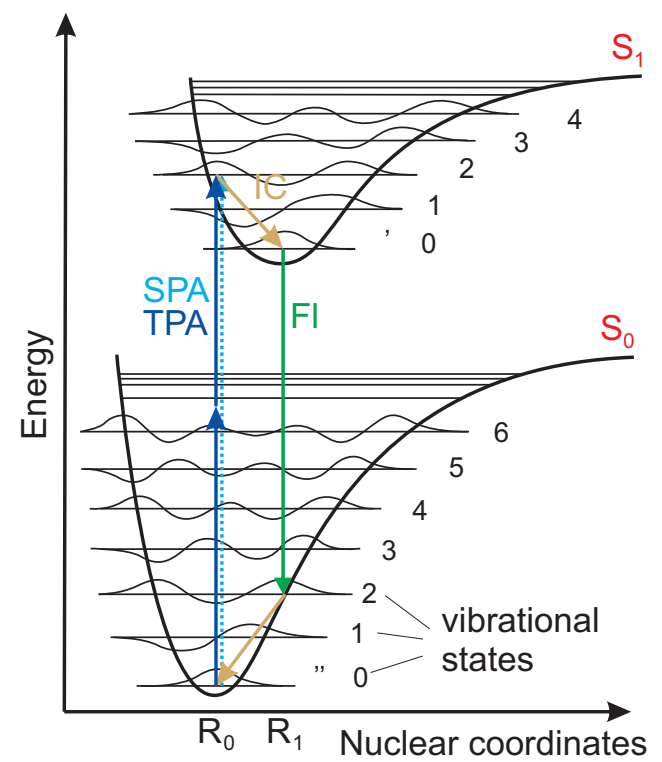

Figure 1: A simplified representation of elementary transitions occurring in single photon absorption (SPA) and two photon absorption (TPA) fluorescence. Fl, fluorescence, IC, internal conversion, $\mathrm{S}_{0}, \mathrm{~S}_{1}$, electronic states.

shift between the excitation and the emission spectrum is called STOKES-shift. Furthermore, single photon excitation spectra tend to be mirror-symmetric with their emission spectra. This is caused by the almost identical vibrational energy gaps in the ground state and the excited state.

The feature in fluorescence that is so beneficial is the STOKES-shift: fluorophores are excited with a certain photon energy, and they emit fluorescence photons with a lower energy. This property can be exploited by the use of appropriate filters which efficiently block the excitation light.

The exceptional sensitivity of fluorescence led to a quest for highly selective fluorescence markers, because the combination of selectivity and sensitivity opens up the possibility to study function in vivo. The ability of the first fluorescent dyes that were applied in the biosciences was restricted to the specific labeling of cell compartments (e.g., 4',6-diamidino-2-phenylindole (DAPI) selectively stains cell nuclei by intercalating with the DNA (Tanious et al., 1992)). Nowadays it is standard to stain individual proteins selectively. General techniques to achieve that are: i) antibody staining. Despite the fact that this technique is comparatively old, it is still indispensable and profited lately from the invention of quantum dots (Lidke and Arndt-Jovin, 2004; Sark et al., 2002). These are extremely photostable fluorescent semiconductors with nanometre size. ii) the coupling of a fluorescent protein to the protein under investigation. The purification of green fluorescent 
protein (GFP) (Shimomura, 2005; Shimomura et al., 1962) won the NobEL prize 2008, and with today's toolbox of molecular engineering kits, new applications are published daily. Additionally, fluorescent markers are used as biosensors to detect $\mathrm{Ca}^{2}$ +-concentrations (Schild, 1996; Tsien, 1988, 1989a,b), other ion-concentrations, $\mathrm{pH}, \mathrm{cAMP}$, and membrane voltages. A nouveau trend is the expression of FRETpairs (FÖRSTER resonance energy transfer, (Förster, 1948)) which act as biosensors: a conformational change that is caused by the binding of the probe which shall be detected leads to a change in FRET-efficiency.

The invention of a big amount of fluorescent dyes is accompanied by advances in fluorescence imaging technique. Efforts are taken to extract molecular parameters from biological samples. With fluorescence recovery after photobleaching (FRAP, (Braeckmans et al., 2003)) it is tried to measure diffusion constants. The same is aimed by fluorescence correlation spectroscopy (FCS, (Gennerich and Schild, 2000; Kim et al., 2007)). Fluorescence lifetime imaging microscopy (FLIM, (Duncan et al., 2004)) is used to measure FRET-signals (Wlodarczyk et al., 2008; Wouters et al., 1998).

The first optically sectioning fluorescence microscopes were single photon excitation laser point scanning devices (Carlsson et al., 1985; Naora, 1955). Time resolution was soon improved by multiplexing (Boyde, 1985; Petráň et al., 1968). The necessity for a confocal pinhole became irrelevant by the use of two-photon excitation (Denk et al., 1990). The axial resolution which is about the size of $\lambda$ in standard sectioning fluorescence microscopes was improved by coherent excitation through two opposing lenses (4Pi-microscopy, (Hell and Stelzer, 1992)). The most recent advances comprise the attempt to optimise the microscope for the extraction of a certain parameter at the cost of others: multispectral systems (Lansford et al., 2001; Tsurui et al., 2000) trade temporal resolution for energy resolution. High temporal resolution systems in fast imaging systems (Wolleschensky et al., 2006) trade spatial resolution. Finally, new fluorescence techniques arouse that break the diffraction barrier which was previously considered a fundamental physical limit. Stimulated emission depletion microscopy (STED) (Hell and Kroug, 1995; Hell and Wichmann, 1994) employs reversible fluorescence transitions and obtains sub-resolution information. Super-resolution is also achieved by photoactivated localisation microscopy with independently running acquisition (PALMIRA) (Egner et al., 2007), where photoswitchable proteins are switched on and off, and their position is determined with nanometre precision. The final image is then a calculated position map. Various structured illumination techniques can be used for optical sectioning (Neil et al., 1997) and for super-resolution (Schermelleh et al., 2008) in widefield fluorescence microscopy. 
The fluorophores, imaging techniques, and microscope systems presented above are used in the attempt to obtain valid quantitative data. Biological questions of today ask for subcompartimental ion concentrations, e.g. the chloride concentration in the cilia of olfactory bulb neurons (Reisert and Bradley, 2005). Spatial resolution on the molecular scale is desired, and this resolution should be obtained noninvasively and in vivo (Westphal et al., 2008). The temporal resolution is demanded to be high enough to resolve action potentials. The high-end fluorescence optically sectioning microscopes of today are multi-parametric systems, in which the underperformance of a single parameter can already lead to a failure in the attempt to extract the desired information, e.g. the presence of molecular interactions or spatial resolution. If one wants to profit from all the favourable benefits which are offered by the new imaging technologies, a thorough calibration and characterisation of the players is required.

A standard way of assessing the characteristics of a fluorescence microscope is the recording of multispectral $\mu \mathrm{m}$-sized beads (Zucker et al., 2007). These are used for alignment (Zucker and Lerner, 2005), colour calibration, chromatic aberration, and detection of intensity variations. In contrast to that submicron-beads are used for measuring point-spread-functions (PSFs) with the aim to determine the system's resolution (Voort and Brakenhoff, 1990). In the latter case it is mandatory to average some dozens of beads to account for low signal-to-noise ratios (SNR). This makes them rather inappropriate for detecting field-dependent variations like they are present in any microscope. Field-dependent variations in intensity and resolution can for instance be detected by recording and analysing an axial image stack of a homogeneous subresolution fluorescent layer, which is therefore a basic tool for image calibration of sectioned images (Brakenhoff et al., 2005; Pawley, 2006).

The aspects presented above are only steps towards the ultimate goal of all calibration and alignment procedures, that is to render a given imaging task platform independent, i.e. to obtain data which is quantitative, comparable, and reproducible. Many comparisons of microscope systems can be found in the literature, most of which present their experimental results with either normalised or arbitrary units (Bewersdorf et al., 2006; Egner et al., 2002; Sandison et al., 1995; Wang et al., 2005). It is quite obvious that a calibration procedure presenting photon numbers and offering a fair comparison of different systems is as yet lacking.

This thesis aims at the development of quantitation strategies in optically sectioning fluorescence microscopy. These strategies shall fulfil two major requirements in order to suffice the needs of the scientific community: first, they shall yield a thorough characterisation of the instrument. The parameters obtained 
from a quantitation strategy need to be quantitative in a way that they can be used for optimising the data acquisition and enable quantitative data evaluation. Second, these strategies need to be easy to adopt in everyday lab routine. A characterisation routine that is so laborious that is does not leave time for the actual experiment is sure to be ignored.

In the first part of the thesis I will describe the development of a quantitative evaluation tool which fulfils the goals mentioned above. After a general introduction into the concepts of resolution and optically sectioning microscopy, I will develop the theory behind the quantitation tool and explain the information content of the assessed parameters on an example.

The second part of the thesis deals with the assessment of a comparatively new microscopy technique called structured illumination microscopy (SIM). Since this optically sectioning widefield fluorescence microscope exhibits certain features that are absent in confocal laser scanning microscopes (cLSM), special tests had to be developed obtaining parameters to tell the user in biology in which cases SIM can be an alternative to cLSM. 


\section{Extended SIPcharts for the Calibration, Evaluation, Optimisation and Quantitation in Optically Sectioning Microscopes}

In this part of the thesis I will present a nouveau method for the evaluation and optimisation of imaging performance of optically sectioning microscopes called extended sectioned image property chart (eSIPchart) analysis. I show how the SIPchart-routine (Zwier et al., 2004) is theoretically substantiated and extended in order to obtain additional parameters. I employ a fluorescent solution for the creation of an eSIPchart and show its equivalence to thin uniform fluorescent layers with regard to the determination of the illumination profile and the field resolution. I will demonstrate that the use of calibration solutions enables an eSIPchartanalysis that contains, among other things, the following: first, it becomes possible to account for wavelength-dependent aberrations. Second, the objective's correction collar setting is optimised for a given imaging task; and third, photon numbers are determined by investigating the photon statistics. This is done independently for each detection channel.

This section is divided into three parts: after a general introduction into the concepts of resolution, optical sectioning, and modern fluorescence imaging techniques, I will explain the theoretical foundation of the eSIPchart and demonstrate its practical relevance for concrete examples by applying eSIPchart-analysis. Finally, I will compare eSIPchart-analyis with other common calibration and characterisation routines and show exemplary eSIPcharts of some standard optically sectioning fluorescence microscopes.

\subsection{Concepts of Resolution and Optical Sec- tioning}

Optically sectioning microscopes have in common that they can resolve object features axially. A major benchmark for microscope evaluation, calibration, and comparison is thus the axial resolution and optical sectioning strength they are 


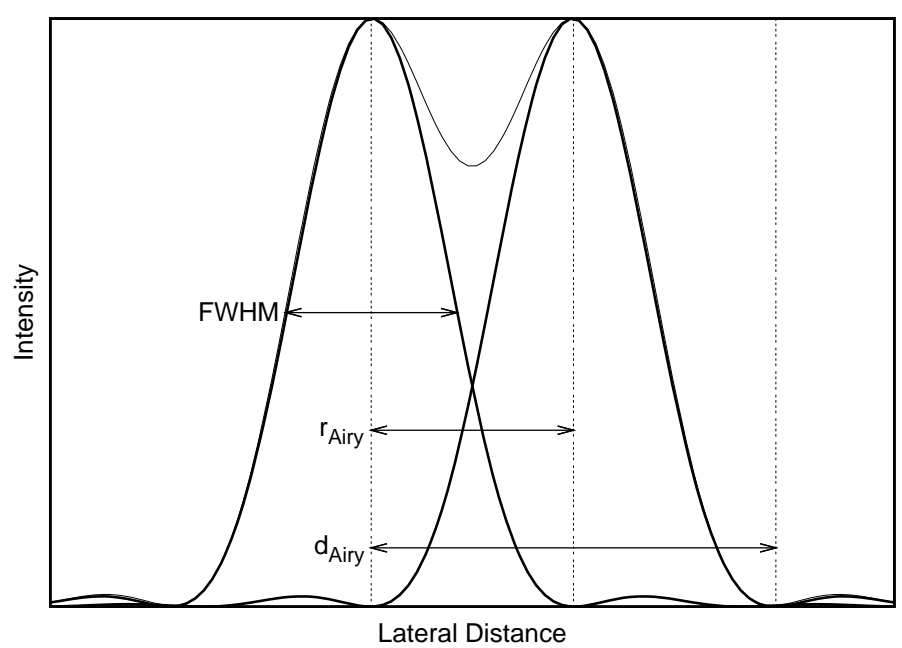

Figure 2: Some definitions of lateral resolution. By definition, two AIRY-patterns are resolved if the maximum of the first disc coincides with the first minimum of the other. The measure for the resolution is then either i) the full-width-at-half-maximum (FWHM), or ii) the radius of the AIRY-disc $r_{\text {Airy }}=0.61 \frac{\lambda}{N A}$, or iii) the diameter of the AIRY-disc $r_{\text {Airy }}=1.22 \frac{\lambda}{N A}$. The envelope reveals the effective intensity distribution.

able to accomplish. Therefore, I will introduce the various definitions that are standardly used for 'resolution' and oppose it to 'thickness of an optical section' and 'depth-of-field'. I present a brief overview over current imaging techniques in optically sectioning fluorescence microscopy. This gives a notion which types of microscopes benefit from characterisation with eSIPcharts.

\subsubsection{Definitions for Lateral and Axial Resolution}

The ability of an apparatus to resolve a feature describes its capability to register two distinct but similar entities as separate. The term 'resolution' describes a measure of the particular distance. In microscopy, the measure can be defined for many dimensions of which space, time, and wavelength are the most important ones. The optical apparatus of a microscope is designed for resolving spatial distances of intensity distributions.

The laws of FRAUnhOFER-diffraction can be used to describe light going through a circular aperture. The lateral far-field diffraction pattern behind an annular aperture is given by:

$$
I_{1}(0, v)=\left(\frac{2 \mathrm{~J}_{1}(v)}{v}\right)^{2},
$$

also known as AIRY-disc. $\mathrm{J}_{1}$, BESSEL-function of first order, first kind; $v=k r \sin \alpha$, dimensionless optical coordinate, $k=\frac{2 \pi}{\lambda}, r=\sqrt{x^{2}+y^{2}}$ (Born and Wolf, 1999). Thus, a microscope's resolution is defined by the distance at which the AIRY-discs 
obtained by the imaging of two equally bright incoherently emitting point emitters can be recognised as separated. The postulated distance is to a certain extent arbitrary since the effective resolution also depends on the contrast that can be generated in a given instrument. The most common definition requires $25 \%$ contrast and demands a coincidence of the AIRY-discs maximum with the first minimum of the adjacent AIRY-disc (cf. Fig. 2). Then, the resolution is given by the radius of the intensity distribution:

$$
d_{\mathrm{r}, \text { Rayleigh }}=r_{\text {Airy }}=0.61 \frac{\lambda}{n \sin \alpha} \equiv 0.61 \frac{\lambda}{N A},
$$

$\lambda$, wavelength; $n$, refractive index; $\alpha$, semi-angle of the beam spread; $N A$, numerical aperture. Eq. 2 is also known as the RAYLEIGH-criterion. Instead of the radius, the diameter of the AIRY-disc is often used as a resolution measure:

$$
d_{\text {Airy }}=1.22 \frac{\lambda}{N A}=1 \text { A.U. }
$$

A.U., Airy unit. The Airy unit is mostly used as a measure for the pinhole diameter $d_{\mathrm{p}}$ of the confocal pinhole in confocal microscopy. Interestingly, the confocal pinhole is in the majority of cases not an annular but a rectangular aperture for technical reasons. The diffraction pattern is then given by $I(0, v)=\left(\frac{\sin x}{x}\right)^{2}$, which is effectively quite similar to Eq. 1. Additionally and for convenience, the full-widthat-half-maximum (FWHM) of an experimentally determined AIRY-disc is taken as a comparative resolution measure: this is described by E. ABBE's famous formula:

$$
d_{\mathrm{l}}=\frac{\lambda}{2 n \sin \alpha} .
$$

Analogous to the lateral diffraction pattern, the axial diffraction pattern is given by

$$
I_{z}(u, 0)=\left(\frac{\sin \frac{u}{4}}{\frac{u}{4}}\right)^{2},
$$

$u=k z \sin ^{2} \alpha$, dimensionless optical coordinate. This is equivalent to the diffraction pattern in $x$ or $y$-direction behind a rectangular aperture. The respective axial resolution is given by:

$$
d_{z}=\frac{2 \lambda}{n \sin ^{2} \alpha}
$$

From this follows that the ratio between axial and lateral resolution is $\propto \frac{1}{\sin \alpha}$, resulting in a focal intensity distribution that is the narrower, the higher the $N A$ is.

The depth of field is related to the axial resolution which is, NYQUIST-sampling provided $^{1}$, a quarter of the axial resolution (Eq. 6). When a scattering or fluorescing

\footnotetext{
${ }^{1}$ i.e. the sampling frequency has to exceed the measured frequency by a factor of $\geq 2.3$ to enable a reconstruction of the measured frequency (Nyquist, 1924, 1928; Shannon, 1949).
} 


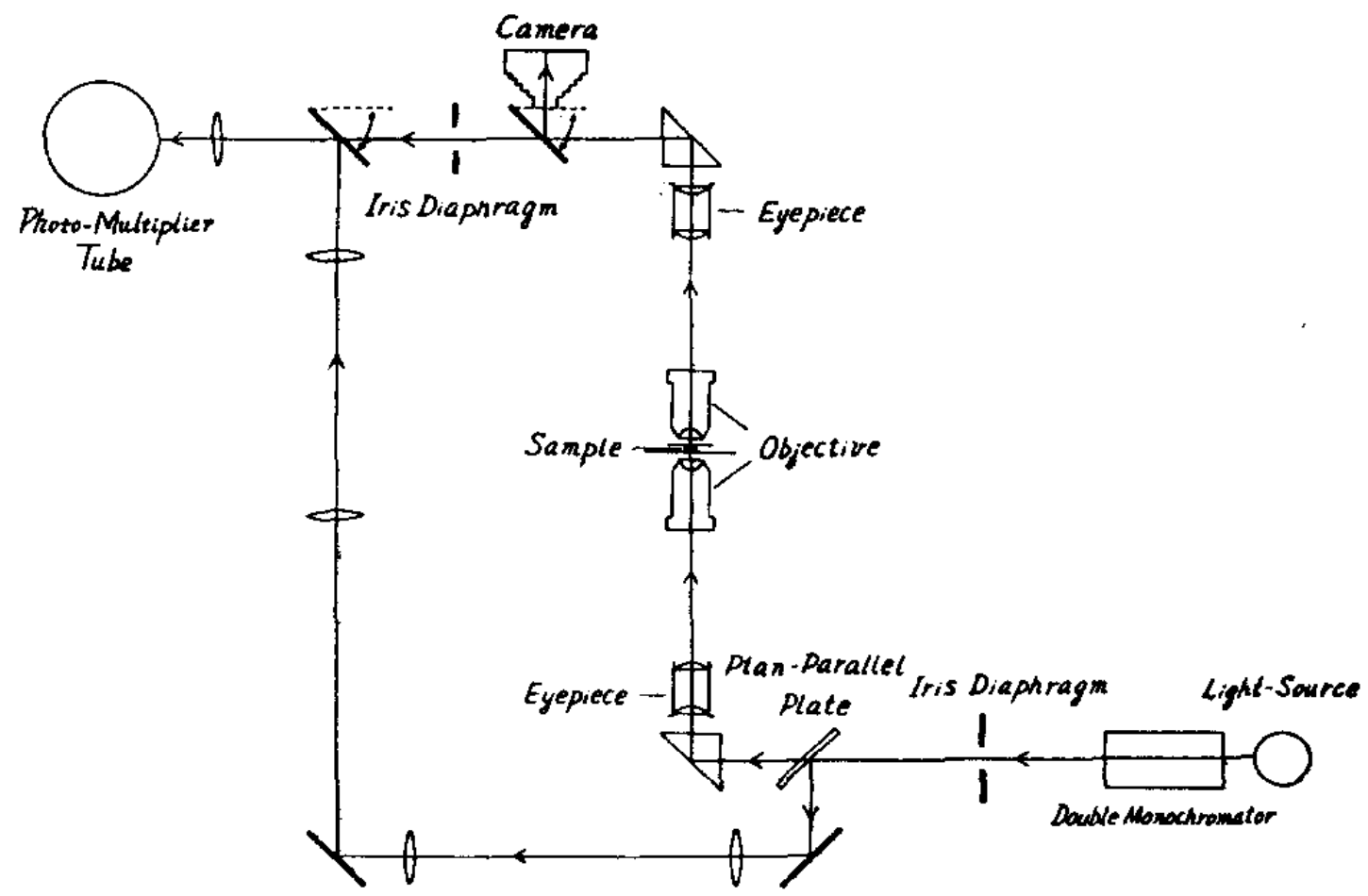

Figure 3: The first microscope with a 'confocal' design was built for microspectrophotometry experiments in 1955 . Unlike today, the sample was moved, not the beam.

object is between the objective and the focal plane in conventional epifluorescence microscopy, it reduces the contrast of the focal diffraction pattern. This out-of-focus contribution to the image can extinguish the focal signal completely so that the nominal axial resolution and depth of field cannot be measured. This phenomenon can be explained by the way in which the optical system treats different spatial frequencies encoded in the object. The finer a structure in the object is, the faster it will attenuate with defocus. The resolution limit will be reached if a structure in focus is too fine to be resolved, i.e., if the objective cannot collect the first diffraction order because its $N A$ is too small. Considering the other direction, the coarser a structure in the object is, the slower it will attenuate with defocus. The limit is a completely featureless structure, e.g. a fluorescence plastic that does not attenuate at all with defocus. Thus, the apparent depth of field in a conventional epifluorescence microscope is much larger than the true depth of field. The low frequency components and out-of-focus blur in an image render the sample featureless. Therefore, a technique is required that rejects the low frequency components as well as the light that originates from out-of-focus regions and thus enables optical sectioning. There are two essential possibilities to do so: first, one could block the central beam in the Fourier plane. Certainly, the mounting of such a filter would create serious artefacts in the image as it would inevitably 


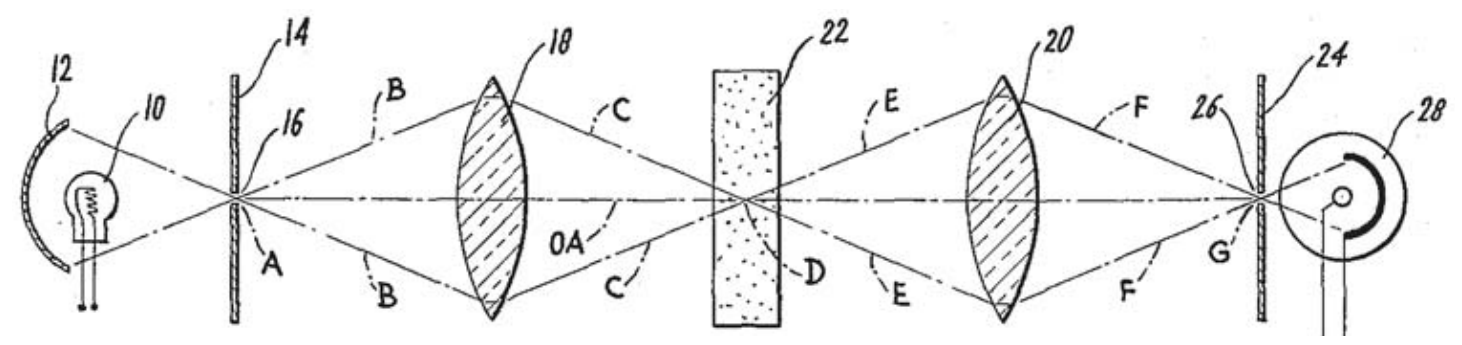

Figure 4: Sketch of the basic confocal principle from the patent script by Minsky, 1961. 16, illumination pinhole; 26, emission pinhole; 18, condenser lens; D, diffraction-limited spot; 22, specimen; 20, objective lens; 28 , photomultiplier tube. The specimen is moved laterally and axially.

block some spatial frequencies in defined directions. The second possibility is to illuminate the sample with a diffraction-limited spot and spatially filter the fluorescence light by a pinhole that is placed in a conjugate image plane where the pinhole has the same diameter as the diffraction-limited spot. This means that all light emerging from out-of-focus planes is rejected by the pinhole. Additionally, very low spatial frequencies are not generated in the first place. This is how it was first done by Naora, 1955 (Fig. 3), although the merits went to Minsky, 1961 who applied for patent in 1957 (Fig. 4). In order to be of practical relevance in biology, this technique of scanning a probe rather than illuminating it wide-field required extraordinary excitation intensities to make it sufficiently fast. It took until the middle-eighties when the availability of lasers and computers made the confocal laser scanning microscopy ${ }^{2}$ (cLSM) feasible for a broader community (Cox and Sheppard, 1983). For the first time in history, it was possible to image 'optical sections' of the sample, where the thickness of an optical section was a function of the confocal pinhole diameter $d_{\mathrm{p}}$. The field of sectioning microscopy has evolved since and has bred a variety of new microscopes that have the capability of producing optical sections.

\subsubsection{Types of Optically Sectioning Microscopes}

Confocal Laser Scanning Microscopes The classical concept of the cLSM has two varieties, namely sample scanning and beam scanning, the first being the most important in the life sciences. In addition to that some sub-categories of confocal microscopy arose that produce optical sections:

\footnotetext{
2The term 'confocal' was first used by Sheppard and Choudhury, 1977.
} 
CLSMs with Improved Spatial Resolution The standard of confocal microscopy is a point scanner, in which the diffraction limited spot of a laser beam is scanned and imaged through a confocal pinhole. Very recent implementations of point-scanning cLSMs that also offer improved resolution are the 4Pi-microscope and the stimulated emission depletion (STED)-microscope. In the former, two opposing objective lenses are used both for coherent illumination and detection. The axial extension of the excitation PSF is reduced by the interference of the two opposing beams, yielding an increase in axial resolution by a factor of $\approx 5$ (Hell and Stelzer, 1992) compared with conventional cLSM. In the latter, the fluorophores in the diffraction limited excitation spot are depleted by a second laser beam that has a torus-like intensity distribution. The effect is that only the fluorophores in the middle of the diffraction-limited spot stay in the excited state, and only their fluorescence is recorded. The lateral resolution of such a system is not diffraction-limited anymore, but principally unlimited (Hell, 1992).

CLSMs with Improved Temporal Resolution The low temporal resolution was a reason for the development of the multifocal YOKOGAWA spinning disc (Petráň et al., 1968; Wang et al., 2005). Here a spinning disc is inserted into a conjugate object plane. The disc is perforated with several thousand holes that are ordered in an ARCHIMEDian spiral of a constant pitch, so that the whole sample is scanned several times with a single revolution of the disc, and a widefield detector like the retina or a CCD-chip can be used. The single holes in the disc simultaneously act as illumination and confocal pinhole. While the transmission of such a disc compared to single point scanners was $\approx 2 \%$ at the beginning, conjugate microlens arrays focussing the excitation light onto the pinholes guarantee a transmission of $\approx 50 \%$ in today's implementations. The temporal resolution is improved by a factor of $\approx 1000$. However, compared with point scanners, the disc scanning approach has three major disadvantages:

1. the thickness of an optical section and the axial resolution are not tunable, because the pinhole size is fixed and usually optimised for high magnification objectives with a high $N A$.

2. much more out-of-focus light is generated and cross-talks to neighbouring pinholes, so the optical sectioning performance is reduced, especially for the imaging of scattering vigorously stained specimens.

3. the fast spinning of the disc creates a considerable angular momentum that will lead to stage vibrations if the disk is not beared with utmost precision. 
Another approach to overcome the low temporal resolution of point scanners is to scan a line and use a confocal slit and/or a line-CCD to achieve confocal imaging (Wolleschensky et al., 2006). This approach suffers from the same resolution and sectioning problems as the spinning disc, but it is more flexible in that the confocal slit width is selectable and multichannel experiments are possible.

Multiphoton Excitation Microscopes A microscopy technique that produces optical sections without the need for a confocal pinhole is multiphoton excitation (Denk et al., 1990). Multiphoton excitation requires extraordinarily high photon flux densities to take place, because otherwise, the probability that at least two photons hit a fluorophore simultaneously is too low. The photon flux density is only in focus high enough to achieve multiphoton excitation. As out-of-focus excitation does not take place, it does not have to be rejected. Restrictions are high costs and slow scanning speed, where the latter can be alleviated by multifocal systems (Andresen et al., 2001; Niesner et al., 2007).

\section{Mathematical Sectioning}

Deconvolution Optical sections can also be obtained by mathematical means, namely by deconvolving the (widefield-) image with the microscope's optical transfer function (OTF), thereby reconstructing the individual origins of the detected photons. Deconvolution unfortunately depends on the exact knowledge of the OTF. While in blind deconvolution certain assumptions about the shape of the OTF are made based on the refractive index, the objective's $N A$ and magnification, this is different in non-blind deconvolution. Here the OTF is experimentally determined by averaging some dozen images of point emitters (PSFs). Both approaches disregard that the OTF is field-dependent, i.e. it varies with distance from the optical axis and with recording depth. Furthermore, deconvolution is highly sensitive to noise.

Deconvolution can also be used to improve the resolution- and optical-sectioningperformance of all kind of confocal microscopes.

Structured Illumination Microscopy Structured illumination is a combination of optical and mathematical means obtaining optical sections: the sample is illuminated with a high-frequency illumination pattern. As this rapidly attenuates with defocus (see above), it serves as a focal label. The pattern is shifted, so that all parts of the specimen are illuminated at least once. From the set of raw images an optically sectioned image can be calculated. This technique will be investigated closely in section 3 . 
In point-scanning cLSM the lateral resolution can be improved in comparison with widefield microscopy by setting the confocal pinhole below $d_{\mathrm{p}}=0.7 \mathrm{~A}$.U. (Wilson, 1990). A 'closed' confocal pinhole $\left(d_{\mathrm{p}} \leq 0.3\right.$ A.U.) yields an improvement in lateral resolution by a factor of $\sqrt{2}$, but is accompanied by a massive loss in signal: at pinhole diameters of $d_{\mathrm{p}} \leq 0.3 \mathrm{~A}$.U., more than $90 \%$ of the in-focus signal is rejected. For this reason, an 'optimal' pinhole setting for applications where resolution is not the most important parameter is considered to be $d_{\mathrm{p}}=0.8-1.0 \mathrm{~A}$.U. Thus one achieves optimal sectioning and SNR performance.

\subsection{Theory and Applications of eSIPcharts}

The meaning of experimental data remains often elusive if an appropriate model is lacking. A model makes data sets comparable qualitatively as well as quantitatively. Additionally, the quality of the model to predict the data can easily be checked. Thus, an inappropriate model can be replaced by an appropriate one which then yields parameters with a physical and/or biological meaning. A major advantage of eSIPcharts compared with its predecessor is its theoretical foundation that enables the extraction of quantitative parameters. This section is dedicated to the presentation of the models that were developed for the creation of eSIPcharts.

\subsubsection{Calibration Standards}

Throughout this thesis the determination of microscope performance was accomplished by using the protocols for sample preparation and measurement specified in this section.

For the measurement of PSFs, InSpeck Green microspheres $(175 \pm 5 \mathrm{~nm})$ with a fluorescence coating ( $\lambda_{\mathrm{ex}}=505 \mathrm{~nm}, \lambda_{\mathrm{em}}=515 \mathrm{~nm}$, ProBes, Karlsruhe, Germany) were used. The experimentally determined PSFs are a result of the convolution of the test object (bead) with the imaging system transfer function. This is always affected by the test object's dimension (Fig. 5). While the PSF's axial extensions are nearly unaffected by this convolution - giving a PSF that is apparently $1.2 \%$ bigger than the real PSF - the lateral extensions are $\approx 1.25 \times$ bigger than the actual microscope's resolution in the given high $N A$-regime. As such they are a compromise with respect to the available signal: if the diameter of the sphere is reduced by a factor $a$, the surface of the sphere will be reduced by $a^{2}$. The beads were dissolved in $70 \%$ ethanol, pipetted onto a cover slip and allowed to dry. Mounting medium (15 $\mathrm{\mu l}$ Component E, Probes, Karlsruhe, Germany) was added. Then it was gently sealed by covering it with an object slide. After the curing of the embedding medium, the preparation will be stable for months if stored in a 


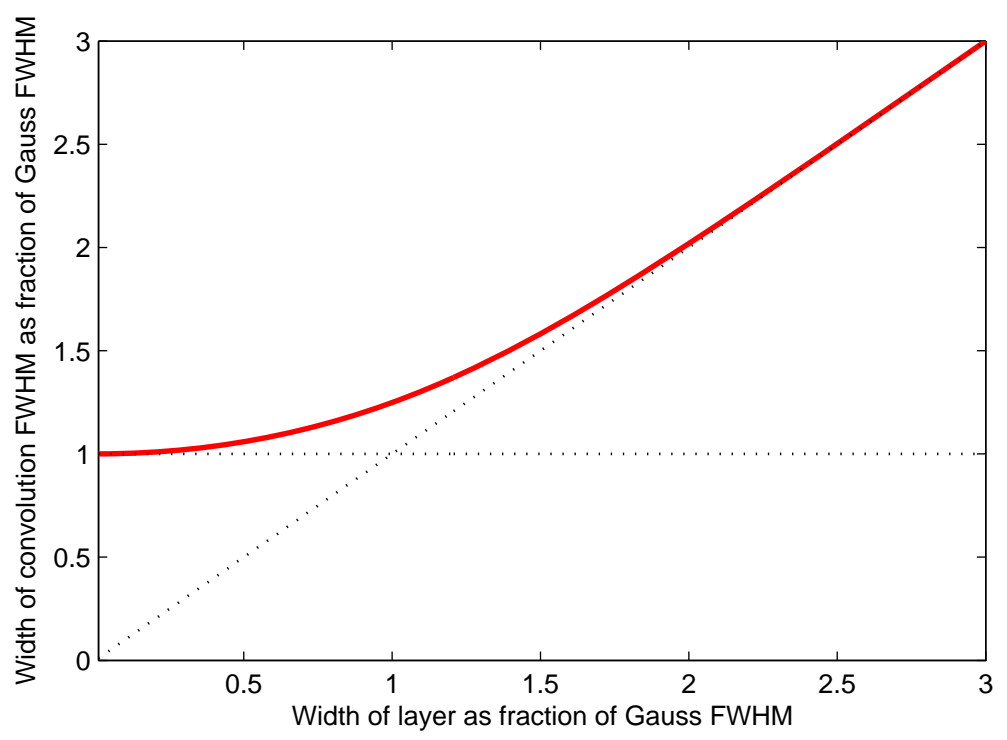

Figure 5: Convolution of the microscope's PSF with extended objects. If the object size is almost zero, the measured dimension (red line) will be the microscope's PSF. If the object size becomes larger, the measured dimension will cumulatively deviate from the PSF's extension until it eventually approaches the object size. Fluorescent beads that have a similar size like the lateral PSF will therefore appear to be $25 \%$ larger in $x y$-direction, while in the direction of the optical axis the difference will only be $1.2 \%$. The systematic error for the measured axial dimension a of thin homogeneous fluorescent layer does not exceed $4 \%$.

dark and dry place.

For the recording of PSFs a region with appropriate bead density was manually selected. The recording parameters were chosen to ensure at least NYQUISTsampling in the lateral dimension where possible. For measurements with the widefield-microscope in section 3 this condition could not be fulfilled due to a fixed pixel size of the CCD-chip of (backprojected) $0.1 \times 0.1 \mu \mathrm{m}^{2}$ (objective: $63 \mathrm{x} / 1.4$ PlanApochromat). The axial stepwidth was generally set to $100 \mathrm{~nm}$. The experimental data was converted into TIFF-files and processed in Matlab: single bead image stacks were manually cropped and GAUssian-filtered with a kernel size of 0.7 . This gentle smoothing alleviates the bias caused by collecting intensity information into voxels: a voxel is a synthetic cuboidal measure that does actually not exist in real space. A 2D-GAUssian fit was applied to the $x y$-plane which contains the centre-of-gravity to obtain lateral resolution data. The $z$-line containing the centre-of-gravity was fitted using a 1D-GAUSSian in order to obtain axial resolution data. This procedure includes a systematic error: not the interpolated plane/line containing the centre-of-gravity is fitted but rather real data directly adjacent to the centre-of-gravity. It is therefore only adequate for sufficiently symmetric beads. 
For representation the beads were aligned with subpixel precision to their centre-of-gravity and then averaged. The resulting average bead was interpolated by using cubic splines. After that it was contrast stretched to 256 intensity levels. Central planes were saved as TIFF-files using the 'hot'-colourmap that ranges from black through shades of red and yellow to white.

To measure how a subresolution homogeneous fluorescent layer in the object plane is imaged, I used a reference layer fabricated as $100 \mathrm{~nm}$ thick fluoresceine/perylenediimid layer, which had a refractive index of $\mathrm{n}_{\mathrm{D}}=1.59$ (Zwier et al., 2004). The layer was imaged using an axial stepwidth of $100 \mathrm{~nm}$. Since a homogeneous layer has no information in the lateral direction that can be resolved, the lateral pixel dimension was usually larger than with NYQUIST-sampling. The stack size was matched to the employed $N A$ to record the whole axial intensity distribution. The recorded data was converted into *.mat-files (proprietary file format of Matlab) and processed using a custom software written in Matlab. Results are combined and presented in eSIPcharts as described in section 2.2.4.

Fluorescent solutions were obtained by dissolving FITC $\left(\lambda_{\mathrm{em}}=520 \mathrm{~nm}\right)$ and ethidium bromide $\left(\lambda_{\mathrm{em}}=595 \mathrm{~nm}\right)$ in aqua dest. Concentrations were adjusted to give similar fluorescence intensity over a wide spectral range. The solution was diluted to sufficiently low concentrations in order to avoid profound pre- and postfiltereffects (e.g. reabsorption of fluorescence photons).

\subsubsection{Modelling the Axial Intensity Response}

A first approximation to the axial intensity profile of a thin homogeneous fluorescent layer is a GAUsSian function:

$$
y=y_{0}+A \exp \left(-\frac{4 \log 2\left(z-z_{0}\right)^{2}}{d_{z}^{2}}\right)
$$

$y_{0}$, offset; $A$, amplitude; $d_{z}$, FWHM; $z_{0}$, axial position. The theoretical description of the axial intensity distribution (Eq. 5) differs from the GaUssian intensity distribution only for values below 10\%. This means that if the FWHM is taken as observable for convenience, there is nearly no difference between these functions. For the eSIPchart-routine I modified at first the fit-function by including a skewfactor $s$ accounting for the fact that the axial intensity profile will not be perfectly symmetric due to slight refractive index mismatch in most cases:

$$
z^{\prime}=z \exp (s z)
$$

Naturally, this way of defining a skew factor empirically creates a bias with respect to the actual axial position, whose effect is however marginal, particularly since $s$ is usually below 0.1 (see Fig. 6). 


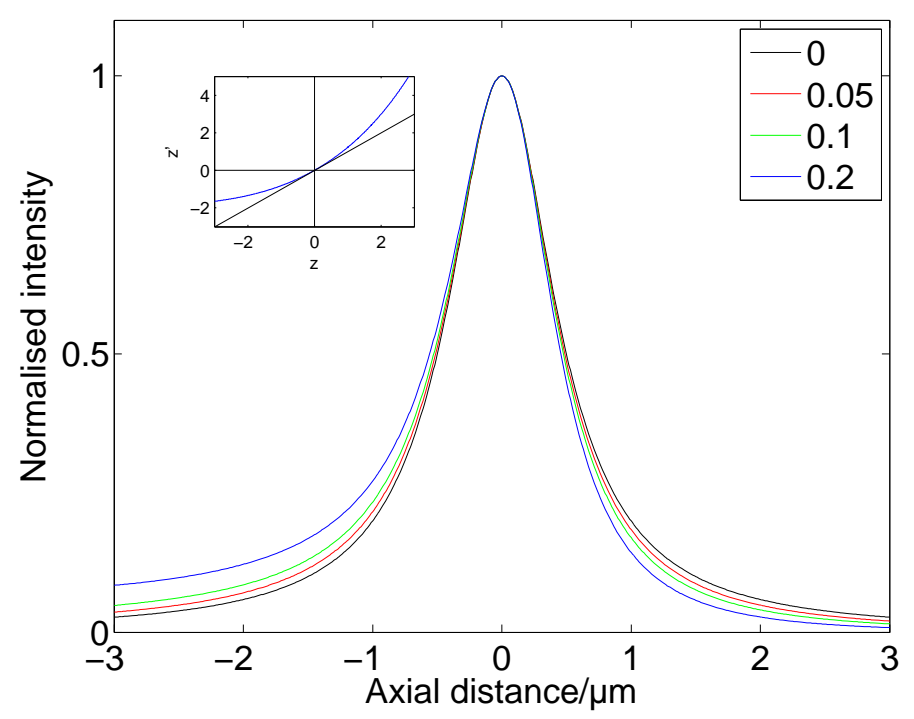

Figure 6: Effect of the skew parameter on the fit function for three different values of the skew $s$. The empirical skew is a good approximation to the data as long as the data does not extend too far in the constant region, and as long as $s$ is sufficiently small. Here, $s$ was generally below 0.1 (green line). The inset shows how a skew of $s=0.2$ changes the axial coordinate.

A GAUSSian function is an appropriate model in cases where the objective's back aperture is homogeneously illuminated. In contrast to that the illumination light source in cLSM is a laser that features a GAUssian intensity profile. In this case the convolution of the excitation PSF with the emission PSF results in an intensity profile that can rather be described with a LoRENTzian function in the axial direction (Qian and Elson, 1991):

$$
y=y_{0}+\frac{2 A}{\pi} \frac{d_{z}^{2}}{4\left(z-z_{0}\right)^{2}+d_{z}^{2}} .
$$

Obviously, a steady transition takes place from an illumination with a GAUssian beam profile that is cut at the back aperture and a homogeneous illumination, making a decision for either model ambiguous.

Taking all this into account, the most adequate description can be obtained from a convolution of a GAUSSian- and a LORENTzian function, known as the VOIGTfunction (Armstrong, 1967; Voigt, 1912). The VoIGT-function was originally used for the description of spectrum lines that are either purely DOPPLER-broadened in the upper atmosphere, or purely LORENTZ-broadened at sea pressure level, or a superposition of these two states. Since its first description in 1912, the VoIGT-function has found broad application in different scientific areas (e.g. X-ray diffraction, Thompson et al., 1987). This is the reason why it has been studied thoroughly. A computationally less expensive approximation of the VOIGT-function 


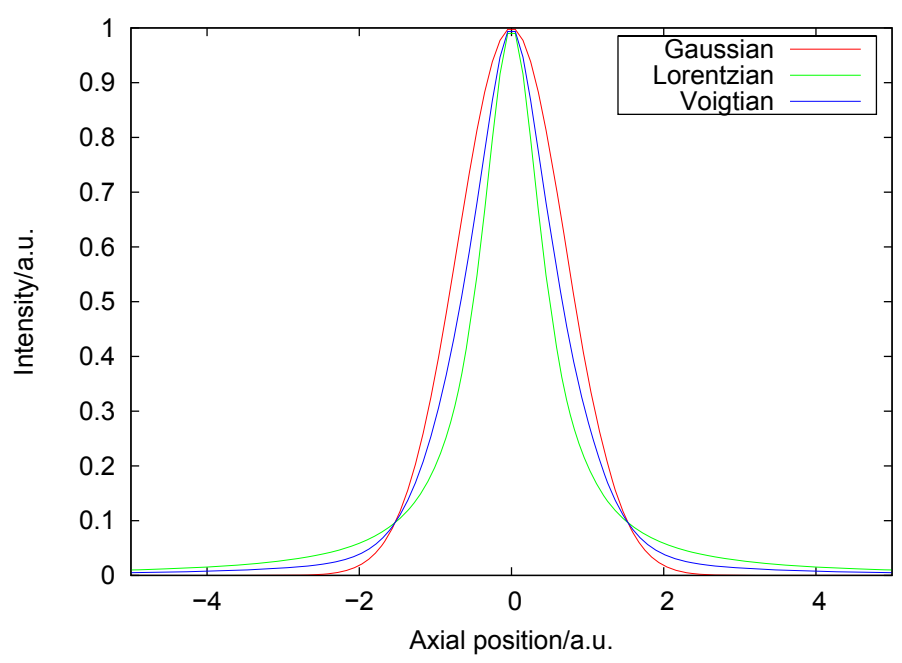

Figure 7: Comparison of the three fit functions employed to fit axial intensity profiles. The Gaussian function is broader at the top and narrower at the bottom than the LoREnTzian function. The VoigT-function can cover any function between these two extremes (here, $m_{u}=0.5$ ). The integrated area under all curves is identical.

is a linear combination of a LoRENTZian and a GAUSSian function with equal width of both functions, known as pseudo-Voigt (Ida et al., 2000; Wertheim et al., 1974):

$$
y_{0}+A\left[m_{u} \frac{d_{z}^{2}}{4\left(z-z_{0}\right)^{2}+d_{z}^{2}}+\left(1-m_{u}\right) \exp \left(-4 \log 2\left(\frac{z-z_{0}}{d_{z}}\right)^{2}\right)\right]
$$

$m_{u} \in[0,1]$ weight factor defined in $\mathbb{R}$. Its systematic error compared with the regular Voigr-function is less than 1\%. For simplicity, I will use the term 'VoigTfunction' throughout the thesis where 'pseudo-VoIGT-function' is meant.

A comparison of the three fit functions employed (Eqs. 7,9 and 10) is shown in Fig. 7. The area under each of the three functions is identical. The GAUssian function drops much faster to zero than the other two functions, but it is broader around its maximum. The Voigr-function can adopt any function form between the GAussian and the LoRENTzian form. The relevance of the VoIGT-function becomes clear when it is compared with the GAUSS- and the LORENTZ-function regarding how they fit real data (Fig. 8). Here, an LSM 510 META equipped with a $40 \mathrm{x} / 1.2 \mathrm{C}$-Apochromat objective was used to record an axial image stack of a thin homogenous fluorescent layer. At $\lambda_{\mathrm{em}}=524 \mathrm{~nm}$, it can be seen that the GAUSSIAN function never quite fits the data. Its residuals systematically deviate from zero. The LoRENTzian function is much better suited to fit the data, although it misses the peak of the axial intensity distribution. The residuals of the VoIGT-function at last nicely strays around zero, and only PoIssonian noise makes them larger in the 


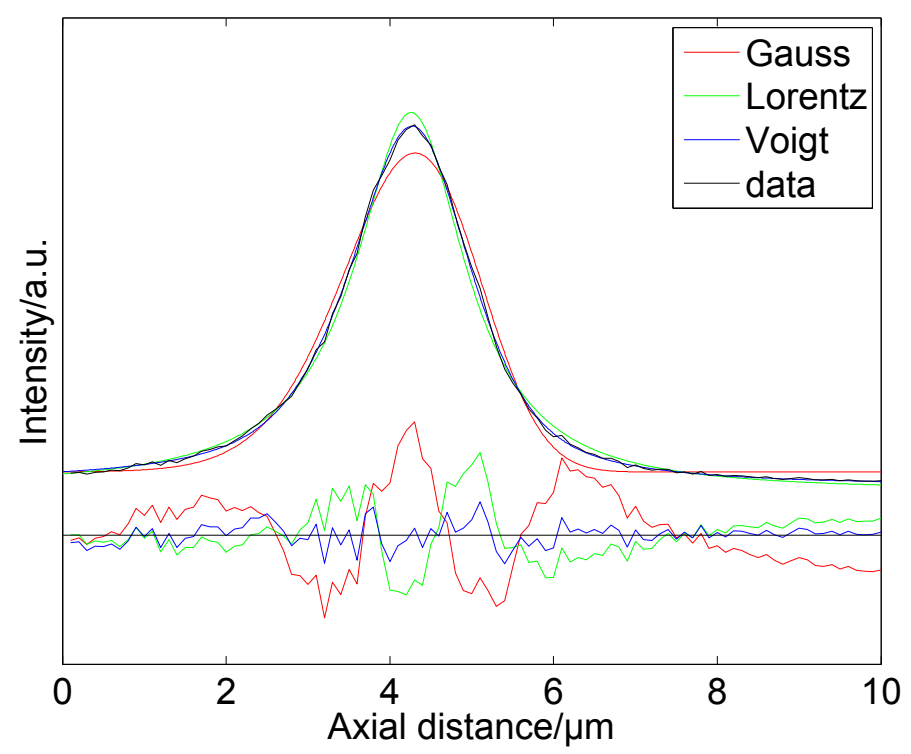

Figure 8: Fit of three functions to experimental data. The residuals (scaled with a factor of four) clearly show that the VOIGT-function suits best to describe the data.

maximum of the intensity distribution. Confirming this qualitative statement, the $\chi_{\mathrm{R}}^{2}$-value as defined in Eq. 18 is 7.76 (GAuss), 2.37 (LORENTZ), and 0.75 (VoigT).

Recording an image stack of a fluorescent dye solution instead of a thin homogeneous fluorescent layer results in the axial intensity profile being the convolution of the aforementioned functions with an exponential function that begins at the cover slip solution interface. The exponential behaviour of the intensity profile in solution is due to a variety of reasons:

- even in solutions with low concentrations fluorescence pre- and post-filtereffects cannot be erased. Scattering and absorption of excitation and emission light lead to intensity decay.

- a slight refractive index mismatch can never be excluded and might lead to signal decay as well as signal increase within the solution behind the cover slip solution interface. In the worst case, refractive index mismatch leads to intensity profiles that cannot be modelled.

While a stretched exponential function might be suited well to incorporate all possible sources of multiexponential behaviour (Benny Lee et al., 2001; Siegel et al., 2003), I decided to implement a monoexponential function into the fit functions to be able to extract a robust decay parameter. The skew as defined in Eq. 8 was not implemented into the following fit functions. The GAUssian approximation to an 
axial intensity profile of a fluorescence solution is given by:

$$
y=y_{0}+\frac{A \sqrt{4 \log 2}}{d_{z} \sqrt{\pi}} \int_{z_{0}}^{\infty} \mathrm{e}^{-4 \log 2\left(\frac{\zeta-z_{0}}{d_{z}}\right)^{2}} \mathrm{e}^{-l\left(\zeta-z_{0}\right)} \mathrm{d} \zeta
$$

$l$, decay constant. The solution to Eq. 11 is given by:

$$
y=y_{0}+\frac{A}{2} \exp \left(l\left(z_{0}-z+\frac{l d_{z}^{2}}{16 \log 2}\right)\right) \operatorname{erf}\left(\frac{l d_{z}-8 \log 2\left(z-z_{0}\right)}{\sqrt{16 \log 2} d_{z}}\right)
$$

Like with layers, the LORENTzian approach for fitting the axial intensity distribution of a solution sample suits better for GAUssian illumination in cLSM:

$$
y=y_{0}+\frac{2 A}{\pi} \int_{x_{0}}^{\infty} \frac{d_{z}^{2}}{4(\zeta-z)^{2}+d_{z}^{2}} \mathrm{e}^{-l(\zeta-z)} \mathrm{d} \zeta
$$

The integral in Eq. 13 cannot be solved analytically. A numerical calculation would require excessive computation time, especially since the function is fitted $>15000 \times$ for the evaluation of a standard image stack. An approximation to Eq. 13 can be achieved by multiplying the exponential function with the integrated LORENTZian function instead of convolving them.

$$
\begin{aligned}
y & \approx y_{0}+\frac{2 A}{\pi d_{z}} \mathrm{e}^{-l(\zeta-z)} \int_{x_{0}}^{\infty} \frac{d_{z}^{2}}{4(\zeta-z)^{2}+d_{z}^{2}} \mathrm{~d} \zeta \\
& =y_{0}+\frac{A}{\pi} \mathrm{e}^{-l\left(z-z_{0}\right)}\left(\frac{\pi}{2}+\arctan \left(\frac{2\left(z-z_{0}\right)}{d_{z}}\right)\right)
\end{aligned}
$$

In some cases it appears that the intensity recorded from a scan into the solution does not drop to zero but to a constant value, although the drop is exponential. For these cases, I added a parameter $e_{\text {off }}$ to modify the exponential function: $\left(1-e_{\text {off }} \mathrm{e}^{l\left(z-z_{0}\right)}+e_{\text {off }}\right.$.

The most adequate description of the axial intensity distribution of a solution is given by the convolution of the VOIGT-function with an exponential function:

$$
\begin{array}{r}
y=y_{0}+A \int_{x_{0}}^{\infty}\left(m_{u} \frac{d_{z}}{4(\zeta-z)^{2}+d_{z}^{2}}+\right. \\
\left.+\left(1-m_{u}\right) \exp \left(-4 \log 2\left(\frac{\zeta-z}{d_{z}}\right)^{2}\right)\right) \mathrm{e}^{-l\left(\zeta-z_{0}\right)} \mathrm{d} \zeta
\end{array}
$$

Like with the LORENTZ-function (Eq. 13), an approximation is obtained by converting the convolution into a multiplication:

$$
\begin{array}{r}
y=y_{0}+A \mathrm{e}^{-l\left(z-z_{0}\right)}\left(\frac{m_{u}}{\pi}\left(\frac{\pi}{2}+\arctan \left(\frac{2\left(z-z_{0}\right)}{d_{z}}\right)\right)+\right. \\
\left.+\frac{1-m_{u}}{2}\left(1+\operatorname{erf}\left(\sqrt{4 \log 2} \frac{z-z_{0}}{d_{z}}\right)\right)\right)
\end{array}
$$

The systematic error made by the approximations in Eqs. 15 and 17 is below $1 \%$ as long as certain experimental conditions are met: 
- the position $z_{0}$ of the cover slip solution interface in the axial image stack should be between $\frac{1}{5}$ and $\frac{1}{3}$ of the whole stack.

- the exponential decay within the solution needs to be small.

The quality of fit models for certain data types can be assessed by using nonlinear least square analysis (NLLS). In order to yield mathematically founded parameters, the data has to follow certain assumptions. In those cases where these assumptions are not fulfilled, NLLS might still yield an estimate of the 'goodnessof-fit', but fails to predict the 'correctness-of-parameters' (Lakowicz, 2006). In the eSIPchart routine some assumptions are not met: the demand that the fit model is a correct mathematical description of the recorded functionality cannot be fulfilled due to these empirical approximations made by implementing the skew $s$ (Eq. 8) and the use of the VoIGT-function (Eq. 10). Notwithstanding, I applied NLLS in order to generate a map of the fit function quality and in order to compare the different fit functions applied (cf. Fig. 8). NLLS analysis was performed by calculating the reduced $\chi^{2}$, that is $\chi_{\mathrm{R}}^{2}$, for each axial pixel stack fitted:

$$
\chi_{\mathrm{R}}^{2}=\sum_{k=1}^{n} \frac{(X-\mu)^{2}}{X v},
$$

$X$, measured data; $\mu$, expected value from the fit model; $v$, number of degrees of freedom.

\subsubsection{Conversion of Arbitrary Detector Units to Photon Numbers}

Many existing photo-detectors do not yield true numbers of actually detected photons but arbitrary detector units (ADU) that are comprised of the detected photons $\phi$ times the detector gain, plus noise from a variety of sources, plus offset. The magnitude of the detector gain can usually be accessed by settings in the acquisition software. This setting however does not always correspond to a photon amplification factor, but for example to a voltage gradient in a PMT, like it is in ZEISS-microscopes. The main source of noise is a PoISSONian noise that is caused by the quantum nature of the photons. Employing the POISSON distribution of the detected fluorescence photons and the property of the POISSON distribution that its variance equals its mean, the true detector gain - meaning the photon amplification factor - can be determined. With that information the number of detected photons can be calculated:

$$
\frac{\operatorname{var}(\mathrm{ADU})}{\mathrm{ADU}}=\frac{\text { gain } \cdot \operatorname{var}(\phi)}{\text { gain } \cdot \phi}=\text { gain. }
$$

Usually, a time series of a defocused image is measured and a pixelwise statistical analysis performed. A dark image is recorded by prohibiting the laser excitation. 


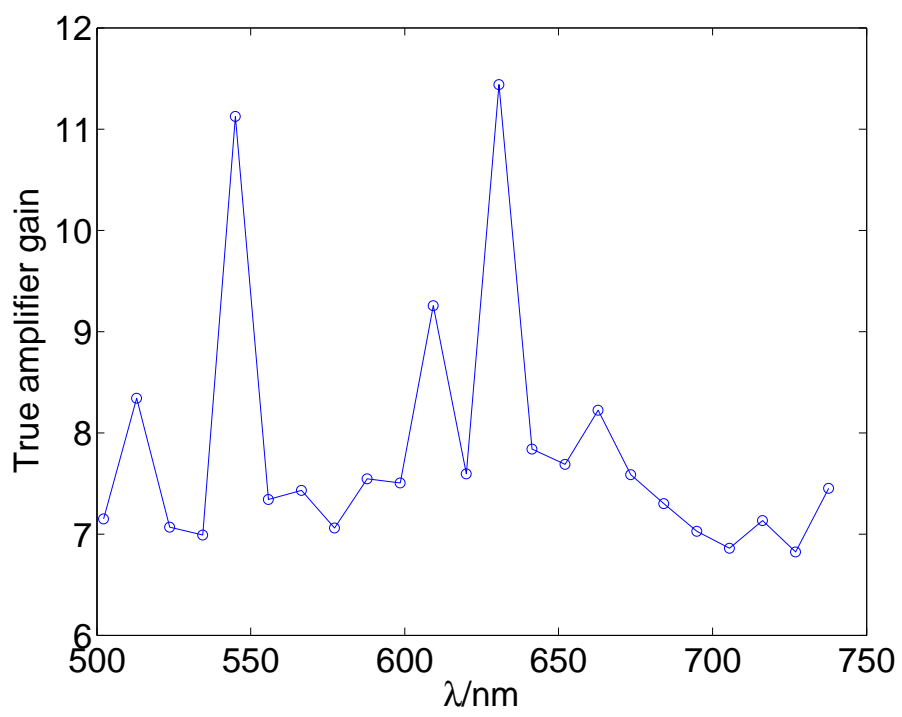

Figure 9: Photon amplification factor emission channel. Although the detector gain was set to ' 670 ' for all wavelengths, the true photon amplification factor is not constant and varies from $6.8-11.5$.

Mean $\left(\phi_{\text {off }}\right)$ and variance $\left(\sigma_{\text {off }}\right)$ determine the origin of the coordinate system, and Eq. 19 becomes

$$
\text { gain }=\frac{\operatorname{var}(\phi)-\sigma_{\text {off }}}{\phi-\phi_{\text {off }}}
$$

The resulting data is linearly fitted to obtain the detector gain. This gain is then used to calculate the number of detected photons per pixel (Lidke et al., 2005). Since such a detection efficiency of the detector is wavelength-dependent, a fixed detector setting will yield different gains for different wavelengths. Therefore, this calibration has to be done on each detection channel. An exemplary calibration curve for one PMT is shown in Fig. 9. The data was obtained on an LSM 510 META system. The detector gain was generally set to 670 . The actual amplification factor ranges from $6.8-11.5$ with a different factor for each detection wavelength. Specific characteristics of the data recording for eSIPcharts make it possible to extract the desired data by another protocol than the one depicted above. For the production of eSIPcharts homogeneous objects are employed. This implies that local homogeneity can be assumed, and the photon statistics can be calculated using laterally neighbouring pixels. This assumption has to be cross-checked by investigating the intensity distribution either of the acquired raw images or in the eSIPchart (see Fig. 10). Structured illumination microscopy as it is described in detail in section 3 can generate artefact stripe patterns, so that local homogeneity is not given. Furthermore, the binning kernel must have an adequate size: if the binning kernel is too large, local homogeneity cannot be assumed for a variety of reasons, e.g. spherical aberrations or an object that is not perfectly perpendicular 
to the optical axis. Despite this, the binning kernel needs to be at least $3 \times 3$ to yield a statistically justified variance. For creating eSIPcharts for data recorded on cLSM systems, the binning kernel was set to $4 \times 4$. In addition to that, the recording of a $z$-stack ensures the mapping of a considerable part of the detector's dynamic range. Another aspect is that if no dark image is provided, the first image of the image stack is assumed to be the dark image. This works only if the first image of the $z$-stack is recorded from well inside the cover slip. If the investigated system is not 'truly' sectioning, meaning that it is not sufficiently free of cross talk like for instance spinning disk and line scanning systems, thin uniform fluorescent layers have to be employed for photon number calibration.

\subsubsection{Imaging Parameters in eSIPcharts}

For the creation of an eSIPchart, the photon statistics of an axial image stack is evaluated as described in the previous section. Then, the data is binned with a freely selectable binning kernel and after that analysed pixel per pixel using one of the fit functions depicted above. The fit parameters are stored and eventually presented in distinct parameter maps in the eSIPchart. Additionally, the eSIPchart contains general information about the setup used and tabulated average data calculated from the aforementioned parameters. In the following, the single parameter maps are explained. A complete eSIPchart is eventually presented in Fig. 21. The underlying data was recorded on an LSM 510 META system, using a 40x/1.2 C-Apochromat objective, laser excitation at $\lambda_{\mathrm{ex}}=488 \mathrm{~nm}$, and a thin uniform fluorescent layer as sample.

\section{Intensity Distribution}

The parameter map of $A$ of the Eqs. 7 to 16 yields the maximum intensity per pixel. The colour encodes the percentage of the maximum intensity. The best objectives available have extended lens systems to correct all kinds of lens aberrations, of which spherical and chromatic aberration are the most important ones. These corrections are often indicated by "Plan" and "Achromat" on the objective barrel. Despite this, objectives are not corrected for a homogeneous intensity distribution. Mostly, they feature a central-symmetric intensity distribution as shown in Fig. 10. Intensity drops of $30 \%$ are not unusual, although the human eye being specialised for edge contrast fails to see the continuous drop by looking through the microscope's eyepiece. Matters will be worse if fine structured biological samples are observed, because it is impossible to know whether it is the fluorophore distribution in the sample or the objective's transmission characteristics that gives a certain image. 


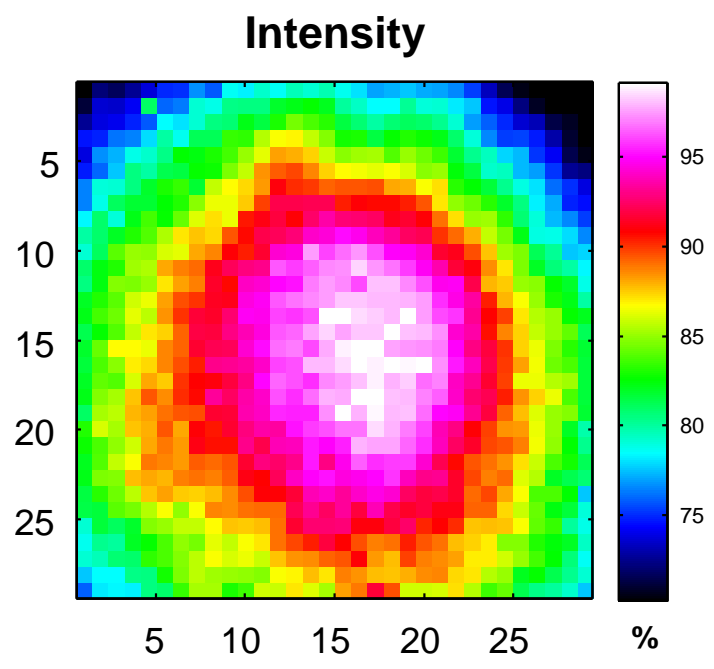

Figure 10: Panel of the distribution of the maximum intensity. In a field-of-view of $230 \times 230 \mu^{2}$, the intensity drops to $70 \%$ of the maximum value. The intensity distribution is central-symmetric, and the maximum intensity in the middle of the field-of-view is indicating that the optical axis is also in the centre.

The intensity distribution gives information about the general alignment condition of an optical system. In the best case, the maximum intensity should be in the centre of the field-of-view, and the intensity distribution should be symmetric. This can be interpreted as one hint among others to a well-aligned system in which the optical axis coincides with the centre of the field-of-view. With the help of the intensity distribution map, irregularities in the homogeneity of the illumination and in-plane objects can be detected easily.

The maximum intensity panel also contains information about the suitability of an objective for a particular imaging task. And it is straightforward to ensure having sufficient significant intensity levels above POISSONian noise with the calculated photon numbers. With the data obtained, a field normalisation can be eventually accomplished (Zwier et al., 2008).

\section{Resolution Distribution}

The parameter map of $d_{z}$ of the Eqs. 7 to 16 yields the axial resolution in FWHM per pixel. Like the intensity, the axial resolution is not homogeneous over the field-of-view, and in the case shown in Fig. 11, it correlates well (but not completely) with the intensity distribution. The position of the maximal resolution is not in the centre of the field-of-view, though. The reason for this can be found in the tilted cover slip (see next paragraph). This makes clear that a check for the position of optimal resolution performance is mandatory for applications where axial resolution 


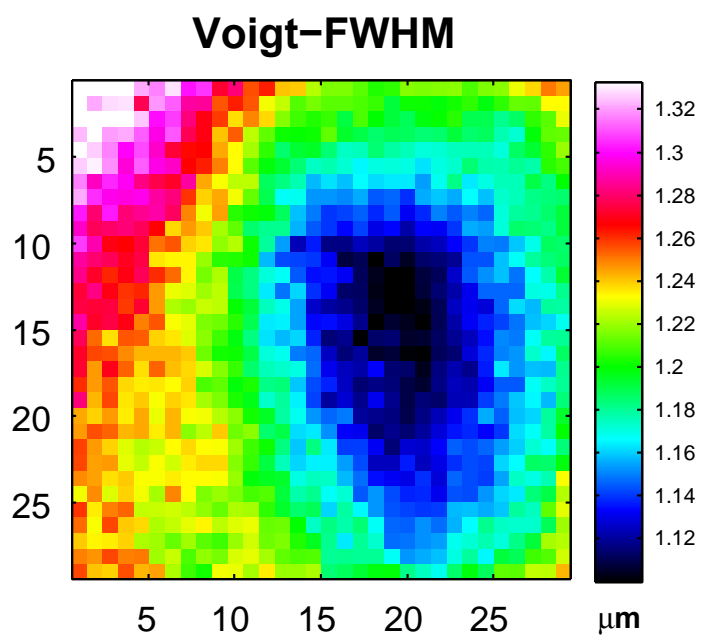

Figure 11: Panel of the distribution of the axial resolution. The resolution distribution is central-symmetric. The measured axial resolution covers a wide range from 1.10 to $1.33 \mu \mathrm{m}$.

is critical, especially in confocal microscopy in which the axial resolution covers a wide range within the field-of-view. The distribution of axial resolution might be used in sophisticated models for field-dependent PSFs in deconvolution. The absolute value of the axial resolution serves as a direct measure for the quality of the alignment and sectioning performance of the microscope.

It is intriguing to realise that the resolution in the field-of-view varies over a considerable wide range. Without the recording of an eSIPchart, the optimal positioning of a sample is somewhat gambling.

\section{Axial Position}

The parameter map of $z_{0}$ gives the surface orientation of the sample relative to the focal plane (tilt). If its range is too high, imaging artefacts arise that can be prevented by the adjustment of the optical table. That being done, $z_{0}$ can be used to reveal spherical aberrations. If the axial position panel shows a linear gradient, over a small range, spherical aberrations can be considered as minor. Any deviation from a linear gradient however clearly indicates spherical aberrations. In this case, an additional surface plot taking the maximum intensity as colour information and $z_{0}$ as height information is a good indicator for the quality of the aberration (Fig. 13). In the present case, the objective does not have nameable spherical aberrations. Conclusively, $z_{0}$ needs to be investigated for steadiness and constancy. 


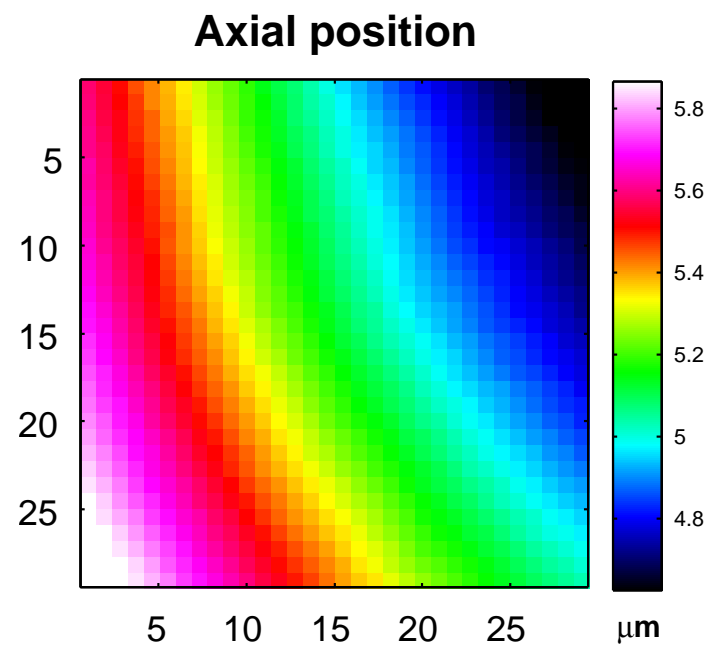

Figure 12: Panel of the axial position of the sample. Here, the angle with respect to the focal plane is only 15', but it is already affecting the resolution distribution (Fig. 2.2.4).

\section{Offset}

The parameter map of $y_{0}$ yields the offset in arbitrary detector units (ADU) per pixel. The offset needs to be positive and should only cover a small range. It contains mainly information about background signal and dark noise. In Fig. 14 the range is $4 \%$ of the signal. Large inhomogeneities can be a hint to stray light entering the system. For pixelated detectors such as CCD-cameras, $y_{0}$ can reveal defect pixels or pixels deviating from the mean performance.

\section{Skew}

The parameter map of $s$ yields the skew of the fit function. If it is constant and nonzero, it can be a hint at either a skewed positioning of the sample on the microscope table, especially in case of water immersion (Arimoto and Murray, 2004), or a refractive index mismatch (Hell et al., 1993). Other possibilities are that it indicates spherical aberrations or a non-optimally adjusted correction collar (Schwertner et al., 2005). The absolute value of the skew reveals the severity of asymmetry. The skew panel shown in Fig. 15 exhibits a comparatively low skew (cf. also Fig. 6) that ranges from $-3.3 \times 10^{-3}$ to $4.4 \times 10^{-3}$. The skew distribution in Fig. 15 is remarkably central-symmetric. This might be a hint at a badly corrected objective or a correction collar that is not adjusted as precise as possible.

\section{Goodness-of-Fit $\chi_{\mathbf{R}}^{2}$}

The numerical value of the reduced $\chi^{2}$ gives an estimation of the goodness-of-fit as already depicted in section 2.2.2: if the eSIPchart analysis is done for several fit 


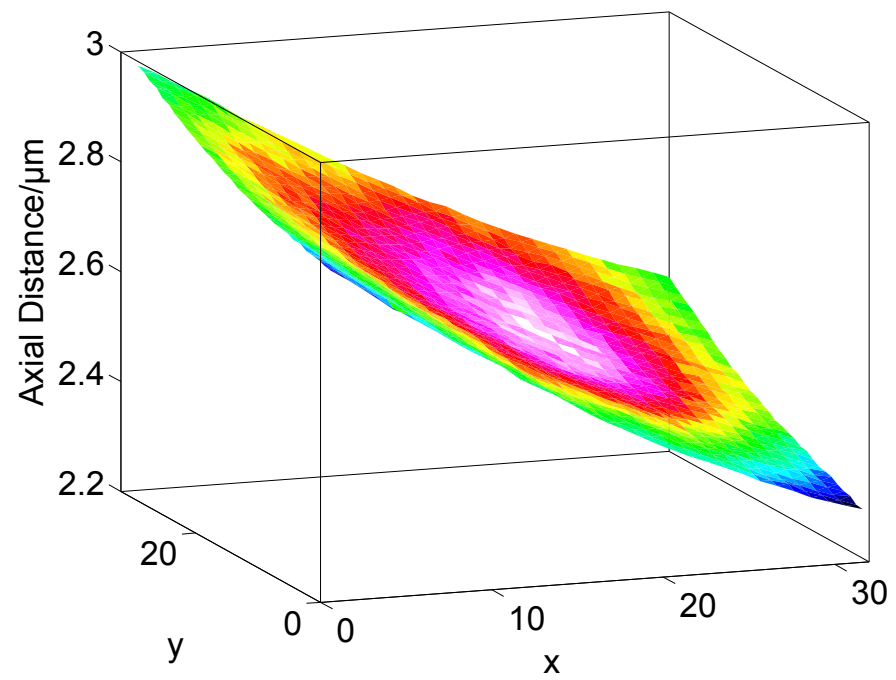

Figure 13: Orientation of the fluorescent layer in the image stack. Here, the flatness of the image reveals that spherical aberrations are negligible. $x$ and $y$-coordinates are severely compressed, the actual angle of the layer to the focal plane is 15 '.

functions, the $\chi^{2}$-map will reveal which one fits best. Using the eSIPchart-routine, it is possible to map $\chi_{\mathrm{R}}^{2}$ for the whole field-of-view in order to detect in which subregions the fit functions do not fit the data best. In Fig. 16, $\chi_{\mathrm{R}}^{2}$ ranges from 1.3 to 6.5. The best goodness-of-fit is obtained in regions where the VOIGT-function is not fully dominated by the LoRENTz-function (cf. Fig. 18). Here, $\chi_{\mathrm{R}}^{2}$ is far from the value that would be considered as 'acceptable' in NLLS, e.g. in time correlated single photon counting (TCSPC) experiments, in which the lifetimes of fluorophores are determined (Gratton et al., 2003). In this case, $\chi_{\mathrm{R}}^{2}=1.17$ is considered an upper boundary for the acceptance of a fit model. For the creation of eSIPcharts this value is not reached. This is mostly due to the approximations that were made for the derivation of the fit functions and it is also caused by the comparatively low number of photons. Thus, $\chi_{\mathrm{R}}^{2}$ as used in eSIPcharts can be regarded rather as a relative measure than as an absolute one.

\section{Decay Parameter}

The decay parameter map of $l$ describes the fluorescence decay of a fluorescence signal behind the cover slip solution interface. If the refractive index is perfectly matched, that is if the correction collar of a water immersion objective is perfectly adjusted, it is expected that $l=0$ (Pawley, 2006). This of course also implies that the fluorescent solution has a low concentration, which avoids scattering and reabsorption effects. The $l$-map reveals information about the penetration depth especially in cases of slight refractive index mismatch. An easy test for whether 


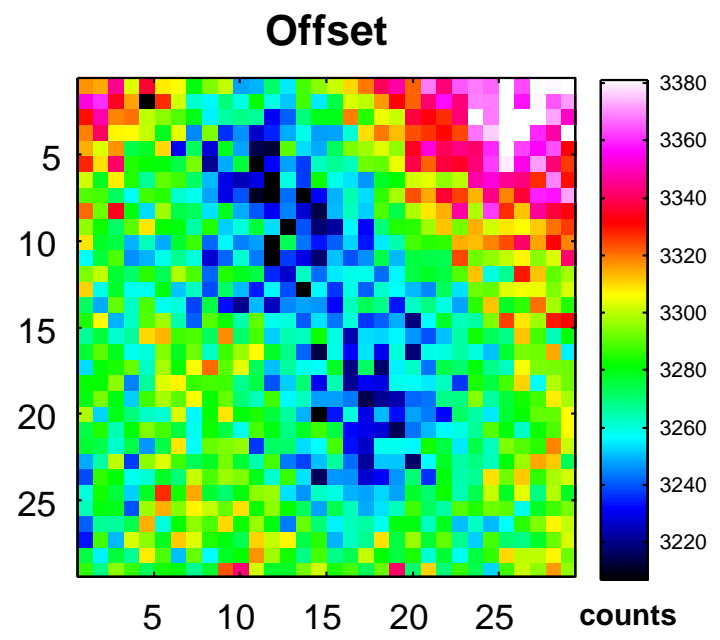

Figure 14: Panel of the data offset. The inhomogeneity reveals minor systematic errors in the fit model applied. It can be neglected due to the small range which the offset covers: it only varies over $4 \%$ of the signal.

the decay parameter is caused by reabsorption and scattering or aberrations and refractice index mismatch is a gentle dilution of the test solution. The decay parameter in Fig. 17 is almost zero and quite homogeneous over the field-of-view.

\section{Weight Factor}

The weight in the VoIGT-function, $m_{u}$, reveals a prevalence for either the GAUssian $\left(m_{u}=0\right)$ or the LoRENTzian function. The former case indicates a homogeneously illuminated back-aperture, whereas the latter stands for the illumination with a GaUssian beam profile (Kuypers et al., 2004). Clearly, in cLSM GaUssian illumination yielding a LoRENTzian axial response function is dominant (Fig. 18) because of the laser illumination. In the present case, $m_{u}$ does not drop below 0.87 . $m_{u}$ cannot be constant in point-scanning cLSM, because the two scanning mirrors cannot be in the conjugate object plane simultaneously: the illumination profile in the back aperture will change necessarily to a certain extent with scanning. The $m_{u}$-map shows whether the assumption of a homogeneously illuminated backaperture is valid, where it is valid, and it reveals artefacts arising from improper mirror alignment.

Because a purely GAUSSian fit function, i.e. $m_{u}=0$, can only be expected for a homogeneously illuminated back aperture, the weight factor panel indicates whether the introduction of a beam expander in the excitation beam path might yield an increase in axial and lateral resolution. I could indeed confirm this effect for a custom-built two photon excitation laser scanning microscope. As a matter of fact, state-of-the-art confocal microscopes are equipped with such an expander. Thus 


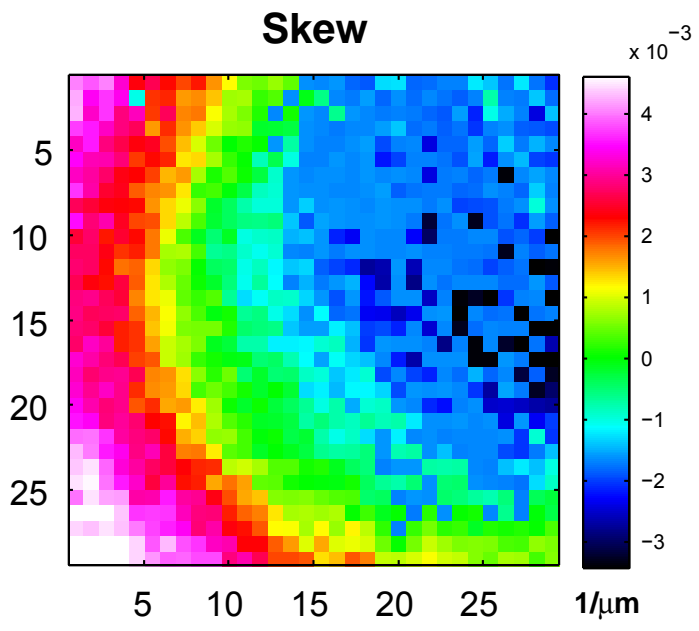

Figure 15: Panel of the skew of the fitted function as defined in Eq. 8. The skew indicates the symmetry of the fit function over the field-of-view. Here, there is a prominent switch from a positive skew via a symmetric to a negative skew.

eSIPcharts help the microscopist to find the optimal expander setting for a given experiment. In experiments in which resolution is important, the expander will be set to maximal settings, whereas it will be set to low expansion in experiments in which light efficiency and speed is important.

\section{Detector Gain}

The determination of the detector gain as it is described in Eq. 19 is essential for the calculation of photon numbers and $\chi_{\mathrm{R}}^{2}$. Fig. 19 shows the variance-over-meanplot with the corresponding linear fit obtaining the gain factor. It can readily be appreciated that the measurement protocol yields a cloud of measured data which is presented here as a 2D-histogram in form of a contour plot. The standard protocol determining the gain factor would yield data points that are straying around a linear function. Here, a nominal detector gain of '600' (amplifier voltage) stands for the true amplification of $\approx 3$. By reason of comparability, there is an increasing demand in advanced microscopy to indicate photon numbers instead of arbitrary detector units.

\section{Data Traces}

The eSIPchart contains a data trace panel for the quick check of the appearance of the data. The panel (Fig. 20) shows five exemplary binned data traces from five positions in the field-of-view. Binning is performed with the same kernel that is used for the calculation of the eSIPchart. The position of the exemplary data traces is calculated so that they are halfway between the corners and the centre 


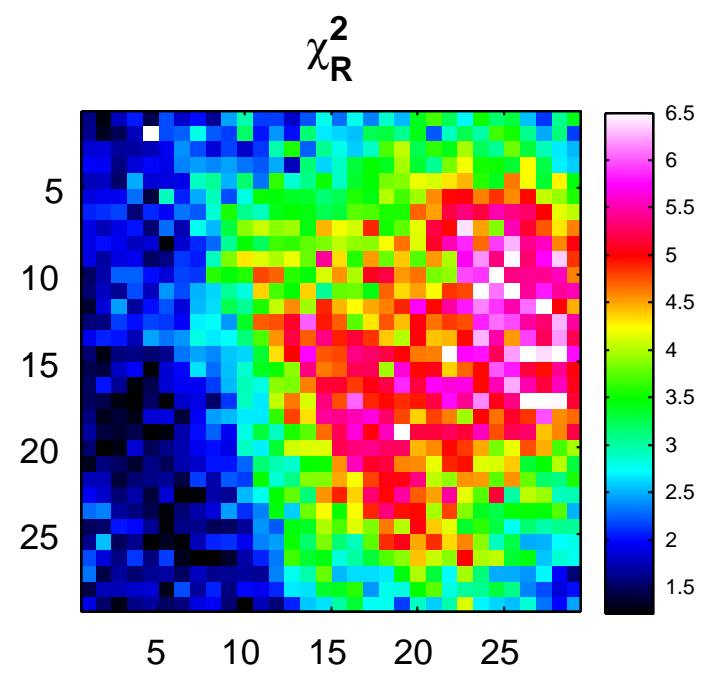

Figure 16: Panel of the goodness-of-fit. Here, the part in the field-of-view that is dominated by a LoRENTZian intensity distribution (cf. Fig. 18) is not fitted to its best.

position of the field-of-view. In addition to that a central data trace is presented. The inset in the data trace panel shows the respective position of each data trace on the maximum intensity panel. Data traces reveal information concerning the quality of the data. A noisy signal indicates the requirement for higher excitation power, longer recording time, higher detector gain, or a larger binning kernel. A similar information is coded in the signal intensity which is given in ADU. The ratio between maximum signal and offset can readily be evaluated, and the settings responsible for the offset can be changed according to that. Obviously, the appearance of the data traces suggests appropriate fit models. In addition to that, a data set containing considerable skew can be identified. It can be seen in the left part of Fig. 20 that the skew goes from negative to positive, and that the central data trace is almost free of skew. Eventually, the tilt of the sample can be monitored qualitatively (Fig. 20, right).

\section{eSIPchart}

The parameters described and mapped in the previous sections are finally gathered for the generation of an eSIPchart (Fig. 21). A complete eSIPchart contains general information about the recording conditions in addition to the parameter maps:

- the microscope system that was investigated. Depending on the microscope system, some options for the data acquisition and evaluation generating an eSIPchart are predetermined: only for 'truly' sectioning microscopes, a fluorescent solution can be an alternative to a thin homogeneous fluorescent layer. Only microscopes that provide a homogeneous illumination of the BFP 


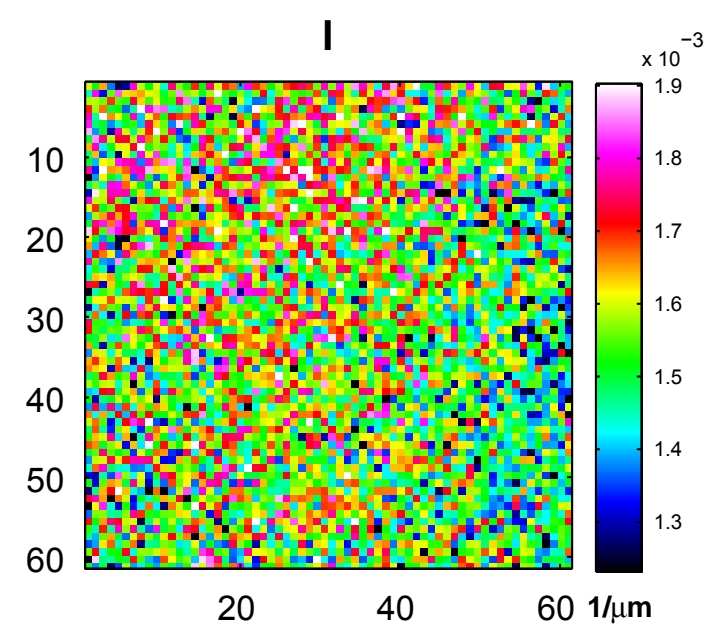

Figure 17: Panel of the decay parameter $l$ obtained from an axial image stack in solution. The decay parameter encodes an increase or decay of fluorescence intensity with increasing imaging depth. Here, it is homogeneous over the field-of-view and very low.

have a GAUSSian axial intensity distribution.

- the objective in use. The objective is a key element in any microscope system, and the concrete shape of many parameter maps can be attributed to the objective in use. Spherical aberrations can be explored directly, and chromatic aberrations can readily be accessed by investigating the eSIPchartparameters in dependence of the wavelength (see section 2.3). The eSIPchart contains the required information to decide whether a given objective is suited for an intended imaging task or not.

- the excitation and emission wavelength. They are important not only for the evaluation of multichannel systems, but also for inter-system comparisons.

- field-of-view parameters and binning kernel. The size of the field-of-view can reveal whether some imaging artefacts that only appear at the edge of the field-of-view are simply clipped. A comparison of the available field-ofview and the respective eSIPchart in different microscope systems and using comparable objectives is intriguing (see for instance Fig. 36 in section 3.2.1).

- the date of the generation of the eSIPchart.

Apart from the system parameter panel described above the fit parameter averages are presented in a tabular form. The tabular presentation allows a quick check for the magnitude of the respective parameter. Unlike in the intensity panel, the average intensity is given in photon numbers. 


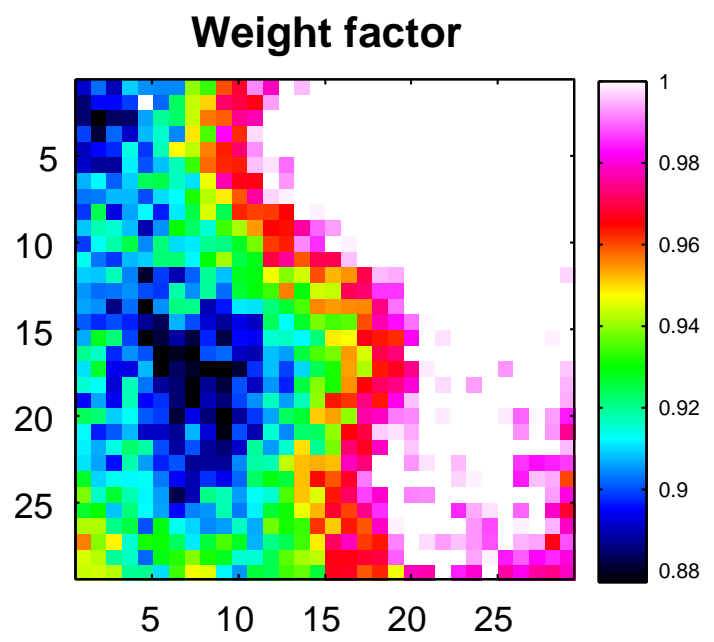

Figure 18: Panel of the weight factor $m_{u}$ in the VoIGT-function. There is a clear prevalence for the LORENTZ-distribution here, which fully dominates the right side of the field-of-view.

The standard deviation of the parameter map is an important measure to assess the homogeneity of the respective parameter. The average of one parameter can be misleading. To give an example, the skew in Fig. 21 appears to be almost zero, and only the comparably large standard deviation informs about the inhomogeneous skew-behaviour.

In this compact form the eSIPchart provides an overview over a big set of parameters that are important in optical sectioning microscopy. With the help of an eSIPchart it is possible to optimise a given microscope for optimal performance in the imaging task to be carried out, and to adjust the respective parameters appropriately. Thus, the recording of an eSIPchart might become an indispensable tool for the calibration and characterisation in optically sectioning fluorescence microscopy.

\subsection{Extended SIPchart analysis}

The parameters determined during the eSIPchart analysis can be used to extend the microscope performance analysis to additional dimensions. One particularly important dimension in fluorescence microscopy of living specimens is the setting of the correction collar of water immersion objective lenses. It is generally held that imaging living specimens with high $N A$ water immersion objectives instead of oil immersion objectives profoundly enhances imaging quality (Sheppard and Török, 1997). The influence of the correction collar that comes with this type of lens is often underestimated for different reasons. Originally, the correction collar was designed to counterbalance spherical aberrations that arise due to cover slips 


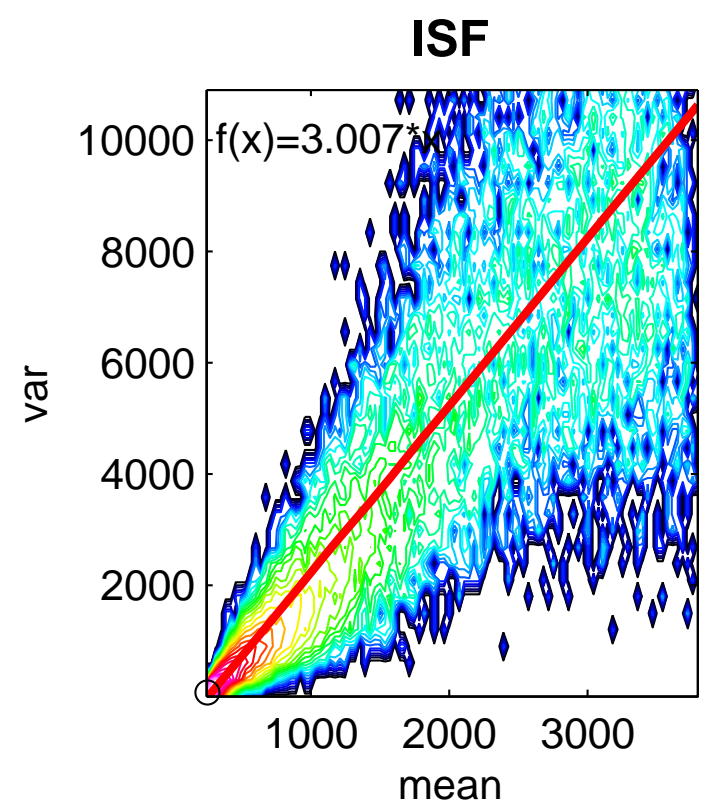

Figure 19: Plot of variance over mean to determine the effective detector gain. Here, a detector gain of 3.007 compared with a nominal detector gain of 600 is ascertained.

whose actual thickness deviates from the nominal one. Water immersion lenses are highly susceptible to this kind of spherical aberration, so that already a slightly tilted cover slip that extends the optical beam path leads to spherical aberrations (Arimoto and Murray, 2004). In theory, it would be optimal to ensure the correct orientation of the coverslip with regard to the optical axis, to determine the cover slip's thickness, and to adjust the correction collar accordingly. In practise however, aberrations arise from more sources than the cover slip thickness. To give some examples, they can arise from slight differences between the refractive index of the embedding medium and the refractive index of the immersion medium, and from the objective's chromatic characteristics.

The correction collar is mostly tuned to yield the highest fluorescence intensity for a given setting. This is tedious to adjust because the focus position changes with the setting of the correction collar. Other approaches use the symmetry of the PSF to adjust the correction collar. Both approaches are badly suited for being based on pure estimation: the human visual system is not designed for the perception of gradual changes in intensity or shape. Clearly, an independent evaluation tool like appropriate software is required.

I assessed the influence of the correction collar by imaging a fluorescent solution that was prepared as described in section 2.2.1. The sample was imaged by using the following settings: microscope system, LSM 510 META; objective, 40x/1.2 CApochromat; field-of-view, $230 \times 230 \mu^{2}, 256 \times 256$ pixels; excitation, $\lambda_{\text {ex }}=488 \mathrm{~nm}$; 

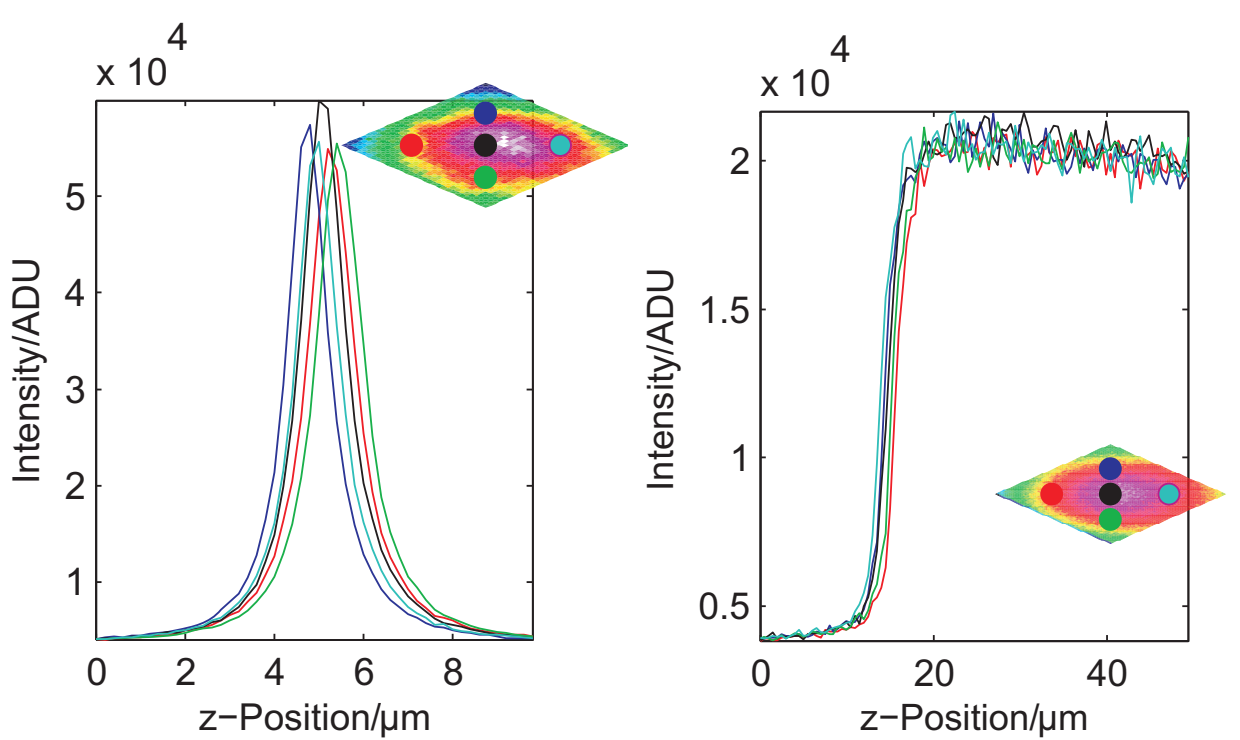

Figure 20: Exemplary axial data traces for five selected positions recorded by using a thin homogeneous fluorescent layer (left) and a fluorescent solution (right). The inset shows the position of the respective data trace on the intensity distribution panel.

pixel dwell time, $3.2 \mu$ s; frame average, 8; detector gain, 670; confocal pinhole, 1 A.U. for the first detection channel; emission, 24-channel $\lambda$-scan from $486-743 \mathrm{~nm}$. The data was binned using a $4 \times 4$ binning kernel, and subsequently fitted using Eq. 16. The averages of the determined parameters were calculated. The intensity and resolution averages of the central region were plotted as a function of the correction collar and fitted using a GAUssian (Eq. 7) for each detection channel. The results are shown in Figs. 22 and 23. In the left part of Fig. 22, the intensity is plotted as a function of the correction collar and the emission wavelength. The colour encodes the emission channels. The spectrum of the fluorescence solution shows two peaks at $\lambda_{\mathrm{ex}}=525 \mathrm{~nm}$ and at $\lambda_{\mathrm{ex}}=640 \mathrm{~nm}$. The data points of the single emission channels are connected by the fit function. This shows that the assumption of a GAUSSian fit function is reflected well in the data. In the right part of Fig. 22, the intensity is normalised and colour coded using the 'jet'-colourmap in Matlab. Between $\lambda_{\text {ex }}=500 \mathrm{~nm}$ and $\lambda_{\text {ex }}=620 \mathrm{~nm}$, a correction collar setting of $\approx 14$ yields the maximum intensity performance. From specifications of the cover slip (\#1.5, Menzel, Germany) one would expect an optimal setting of $\approx 17$. For longer emission wavelengths the demand to adjust the cover slip increases linearly, reaching a setting of $\approx 18$ at $\lambda_{\mathrm{ex}}=738 \mathrm{~nm}$. From this follows clearly that for multichannel experiments a compromise has to be found for the correction collar setting since this cannot be changed during the experiment. In addition to that, this particular example exhibits an intensity loss of $30 \%$ for wrong correction collar settings. 

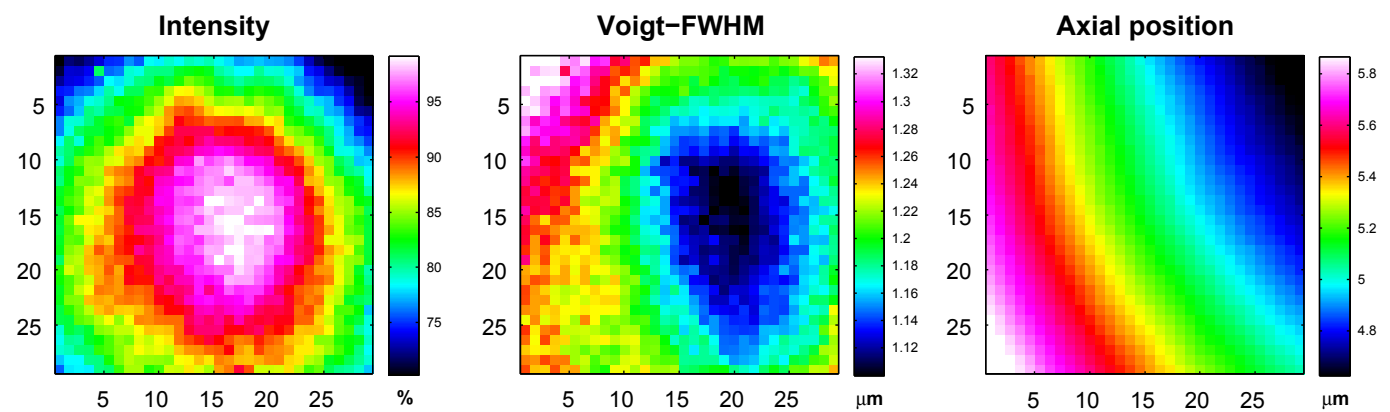

\section{eSIPchart}

Axiovert 200M/LSM 510 META

Objective: C-Apochromat 40x/1.2 W corr

$\lambda_{\mathrm{ex}}=488 \mathrm{~nm}, \lambda_{\mathrm{em}}=534.38 \mathrm{~nm}$

Field of view: $230 \times 230 \mu \mathrm{m}^{2}$, bin $4 \times 4$ from $128 \times 128$ Pixel.

\begin{tabular}{|llll|}
\hline & Average & \multicolumn{1}{c|}{ SD } & \multicolumn{1}{c|}{ Unit } \\
\hline$I_{\max }$ & $1.1 \mathrm{e}+003$ & \pm 84 & Photons \\
\hline FWHM & 1.2 & \pm 0.054 & $\mu \mathrm{m}$ \\
\hline $\mathrm{z}_{0}$ & 5.2 & \pm 0.32 & $\mu \mathrm{m}$ \\
\hline Offset & $3 \mathrm{e}+003$ & \pm 36 & ADU \\
\hline Skew & $1.1 \mathrm{e}-005$ & \pm 0.002 & $1 / \mu \mathrm{m}$ \\
\hline$\chi_{\mathrm{R}}^{2}$ & 3.4 & \pm 1.4 & \\
\hline
\end{tabular}
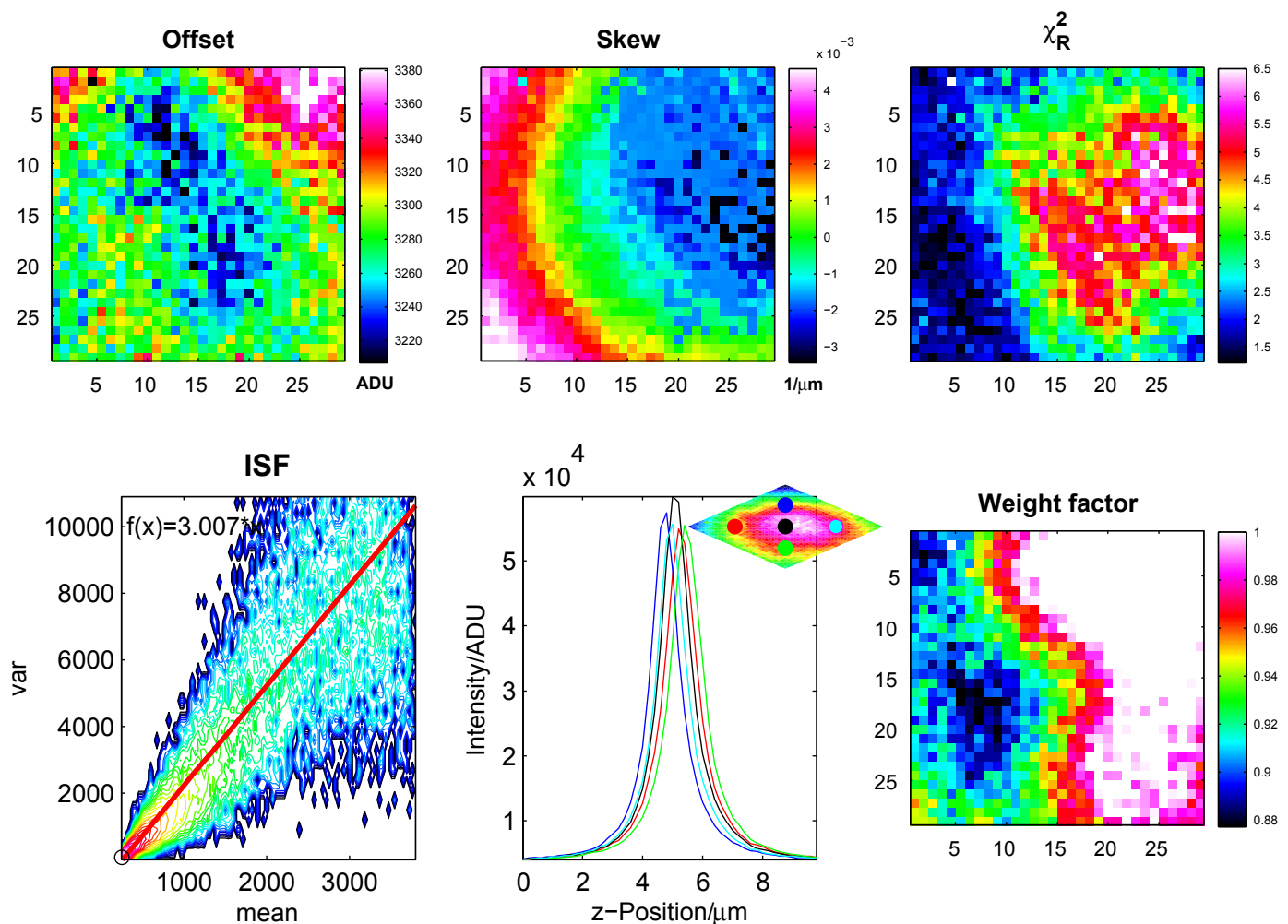

Figure 21: eSIPchart gathering the panels that were shown in the previous Figs. 10-20. The eSIPchart in addition contains information about the system and recording conditions. The panel averages are clearly presented in a tabular form. Mostly, the different panels reveal a distinct inhomogeneity of the parameter behaviour in the field-of-view. Hence the averages have to be looked upon with great care, and special attention has to be laid on the standard deviation. Consider e.g. the skew in this eSIPchart: its average is virtually zero, but its single values range from $-3.5 \times 10^{-3}$ to $4.5 \times 10^{-3}$, resulting in a standard deviation of $2 \times 10^{-3}$. 

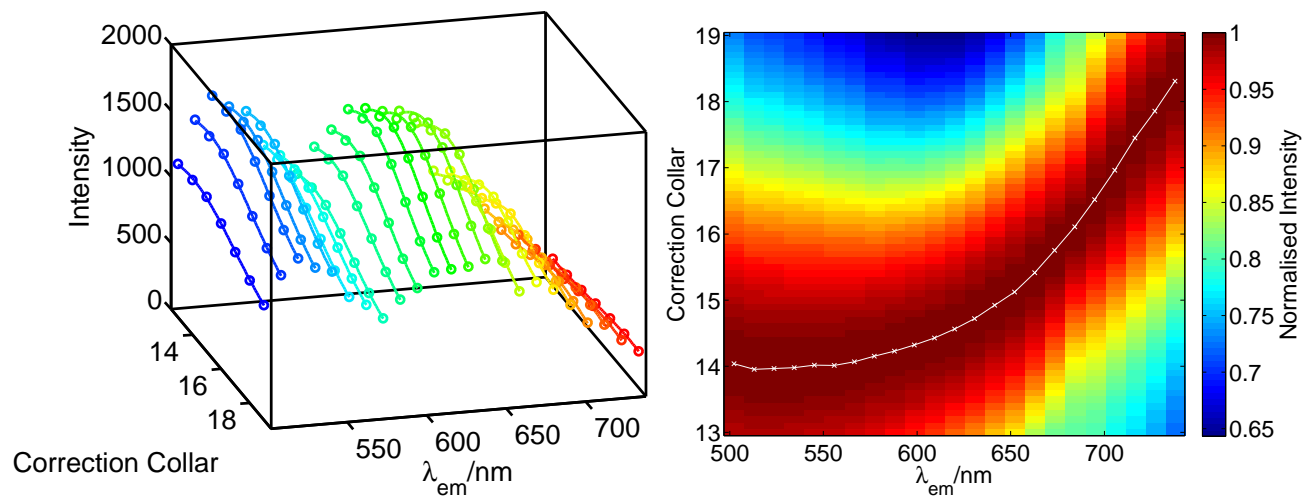

Figure 22: Intensity as a function of the correction collar and the emission wavelength. Left, average intensity as it depends on the emission channel wavelength and the setting of the objective's correction collar. The colour encodes the different wavelengths. Right, the normalised and colour coded intensity as a function of correction collar and emission channel wavelength shows that for optimal transmission at a given excitation channel, the correction collar has to be adjusted accordingly.

Fig. 23 shows the colour-coded resolution as a function of the correction collar and the emission wavelength. Unlike the intensity the resolution is optimal for one setting of the correction collar, with the exception of near-infrared emission. The best resolution for all emission wavelengths from $500 \mathrm{~nm}-700 \mathrm{~nm}$ is obtained by setting the correction collar to $\approx 15$. When the first and the last two emission channels are neglected, the resolution is almost constant. Its absolute value is between 1.5 and $1.7 \mu \mathrm{m}$. According to imaging theory, a linear dependency from the wavelength might have been expected. However, resolution in the cLSM depends on the excitation rather than on the emission wavelength, and the excitation wavelength stays constant. Concerning the theoretical axial resolution limit for small objects (Eq. 6), we obtain $d_{z \text {,theory }}=904 \mathrm{~nm}$ for the given excitation wavelength and $N A$. For a maximally extended object as it is represented by a fluorescent solution, this value could certainly be obtained only with a homogeneously illuminated BFP and with a maximally closed confocal pinhole. It is intriguing to see that a falsely adjusted correction collar can lead to a resolution that is $>1.5 \times$ worse than the optimal one. If the correction collar was adjusted following the general assumption that the maximum intensity reflects the best resolution, one would obtain a loss in resolution of $\approx 30 \%$ in the present case.

The comparison of the optimal correction collar setting for maximal resolution and fluorescence intensity reveals that they do not coincide. This poses no severe problem for $\lambda_{\mathrm{em}}=500 \mathrm{~nm}-660 \mathrm{~nm}$ in practise, but beyond that, a decision for the most important imaging parameter has to be made. 


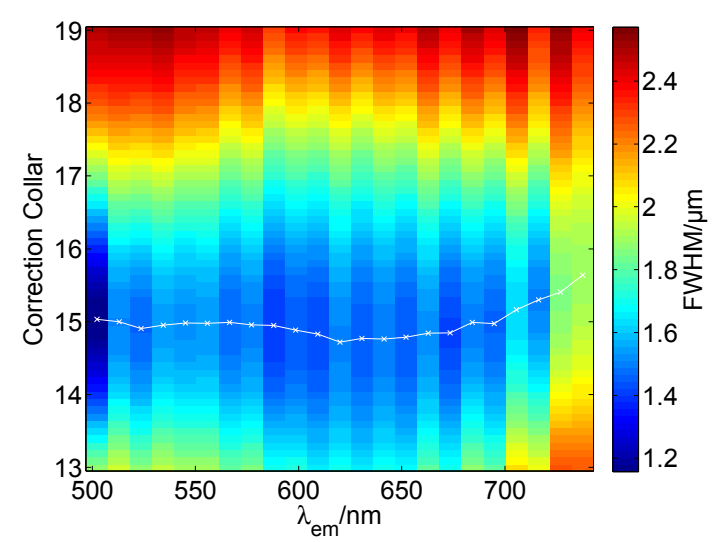

Figure 23: Chromatic aberration in multichannel systems: dependency of the axial resolution on the setting of the objective's correction collar and the emission channel. The best resolution is obtained for a correction collar setting of $\approx 14$, and the resolution is almost constant over the investigated spectral range.

The previous two paragraphs revealed how influential and important an optimised setting of the correction collar is in particular for multichannel experiments. Since there are so many factors that contribute to imaging performance, and since refractive index mismatch becomes relevant already for changes in the third digit, imaging with oil immersion objectives also benefits from thoroughly adjusted correction collars if available.

eSIPchart analysis is even more powerful: with its help the chromatic aberration of a microscope system can be quantified directly. Therefore, it is required to plot the axial position $z_{0}$ over the emission channel wavelength, which of course suffices only for one setting of the correction collar. In order to get a valid measure of the chromatic aberration with respect to all correction collar settings, the true axial position has to be calibrated as reference point. For this purpose, I measured the reference $z_{0}$-position directly by using the reflection of the excitation from the cover slip solution interface. Such a calibration yields Fig. 24. The colour in the figure stands for the different correction collar settings, varying from 13 (blue) to 19 (red). Measured over the whole spectral range, the chromatic aberration can amount to $1.5 \mu \mathrm{m}$, which is in the order of magnitude of the axial resolution. If the spectral detection band is reduced, the chromatic aberration will not exceed $\frac{1}{4}$ of the axial resolution, which is acceptable. 


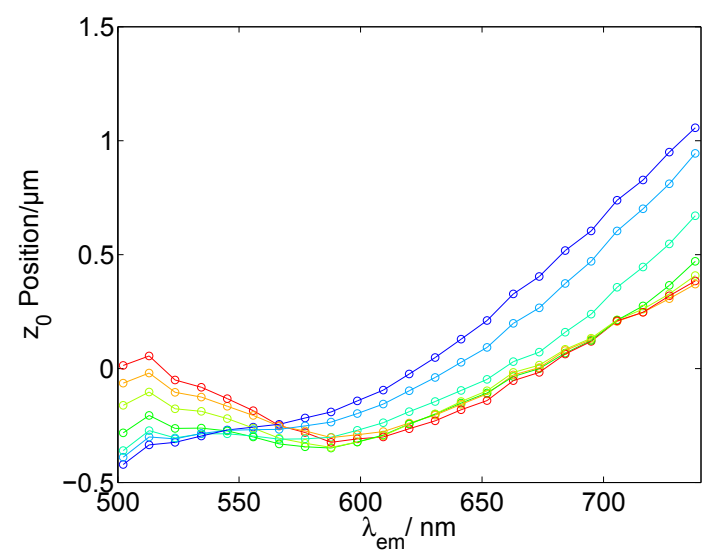

Figure 24: Chromatic aberration in multichannel systems: dependency of the virtual focal plane on the emission wavelength. Colour codes stand for different correction collar settings varying from 13 (blue) to 19 (red). The chromatic aberration is below the axial resolution limit and becomes severe only for emission channels in the near-IR.

\subsection{Discussion}

\subsubsection{Quantification}

An eSIPchart includes a quantitative analysis that calculates photon numbers from the photon statistics of the recorded data. This makes it possible to compare different settings and microscope systems quantitatively. If the same sample is used, eSIPchart analysis will provide absolute measures to assess comparatively:

- the excitation power required to achieve equal fluorescence intensity.

- the SNR for equal fluorescence intensities.

- the signal-to-background ratio.

The calculated gain factor can be used to estimate the number of photons in a biological experiment. Since the photon number is POISSON-distributed, the number of significant intensity levels in the final image can be determined by calculating the square root of the maximum of the photon number.

The determination of the gain factor follows a protocol that differs from the standard. Local homogeneity is assumed to justify a lateral spatial averaging rather than a single pixel temporal averaging. Strictly speaking, this procedure will only be appropriate if i) the sample is indeed homogeneous, ii) the sample is oriented almost perfectly perpendicular to the optical axis, iii) the binning kernel is large enough to yield robust statistics, but small enough to exclude field-dependent variations. Especially the second point will have a big influence on the actually determined gain factor if thin homogeneous fluorescent layers are used as test 


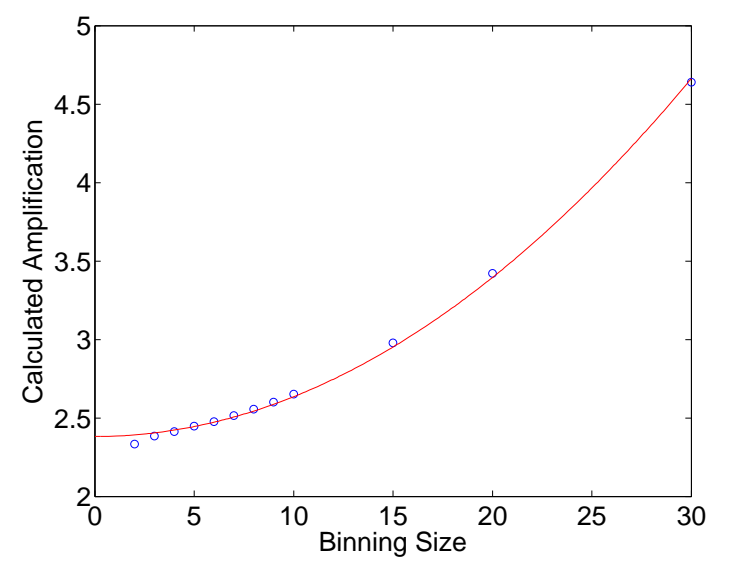

Figure 25: Dependency of the calculated gain on the size of the binning kernel. The binning kernel is given as cross section, i.e. $5 \hat{=} 5 \times 5$ pixels.

object. An example of how the gain depends on the binning kernel size is depicted in Fig. 25. The abscissa is scaled in binning kernel size, i.e. $5 \widehat{=} 25$ pixels. Clearly, there is an unexpected linear dependency between apparent calculated amplification and pixel number. The reason for that is the tilted sample. Consider for instance a severely tilted sample that is only in focus in the centre of the field-of-view. In this case, a large binning kernel acts as a cut through the sample. Fortunately, the relationship shown in Fig. 25 can be used to extrapolate the data to a single pixel. In doing so, an almost optimal gain factor can be determined, which would otherwise require laborious additional measurements. The effect that a tilted sample leads to inhomogeneity is much more pronounced for the measurement of thin homogeneous fluorescent layers than for fluorescent solutions. Provided that the fluorescence intensity measured inside the solution is almost constant, that is $l=0$, local homogeneity can indeed be assumed. For small binning kernels, the adversary effect of a small tilt at the cover slip solution interface is negligible.

\subsubsection{Fluorescent Solution as a Calibration Standard}

The transition from a thin homogeneous fluorescent layer to fluorescent solution for eSIPchart analysis has some profound advantages: most important, anybody can produce a fluorescent solution from the dyes he is using standardly. To my knowledge thin homogeneous fluorescent layers are not commercially available. They are considerably difficult to manufacture with respect to homogeneity and thickness, and it is mandatory to have expertise in spin-coating (Schrader et al., 1998; Zwier et al., 2004). Model and Blank, 2008 fabricated custom layers by letting fluorescent dye dry on the cover slip surface. This is not the best choice 
since such a procedure will result mostly in remnant inhomogeneous fluorophore distribution, and as a consequence inhomogeneous fluorescence intensity. Previously, fluorescent dyes dissolved in polyethyleneimine were sedimented on a cover slip to produce a thin homogeneous fluorescent layer as a tool for the production of SIPcharts (Vicidomini et al., 2007). This seems to be a promising alternative for the custom preparation of thin layers, since the authors claim uniformity, bleaching resistance, and ease of production for these layers. Despite those facts, these layers still lack some important features required for the optimisation of sectioning microscopes, which can easily be included in fluorescent solutions. There is a strong demand for calibration standards that cover a high spectral bandwidth and a high refractive index bandwidth from 1.333 (water) to 1.52 (oil). The recording of fluorescent dye solutions in combination with eSIPchart analysis is such a tool. Concerning fluorescent solutions, they can be manufactured easily so that they cover a large spectral bandwidth. They can be used readily for the analysis of chromatic aberrations in multispectral optically sectioning systems, which was demonstrated in the previous section. Fluorescent dyes can be selected to be soluble in the solution that matches the refractive index of the specimen best. Then it is straightforward to optimise the microscope system and the objective for a particular imaging task. A concentration series analysed with eSIPchart makes it eventually possible to calibrate a given system for concentrations of the dye that is used in a particular biological experiment.

Of course, using fluorescent solutions instead of thin homogeneous fluorescent layers has some disadvantages. I will discuss a few here:

- some fluorescence dyes tend to precipitate at the cover slip which will result in an intensity hump at the cover slip solution interface. As the modelling of this hump would introduce several more parameters in the fit model, such a condition should be avoided by using only freshly prepared solutions for these dyes.

- if multidimensional image stacks are acquired, there is a chance that some water will evaporate from the solution. This increase in dye concentration falsifies the data. For long-term acquisitions and in warm surroundings, the sample should be sealed.

- some fluorescent dyes might be either expensive or toxic or both and as such not convenient as standard probes.

- some fluorescent dyes bleach rapidly in aqueous surroundings. A fluorescent solution has the advantage that bleached molecules are almost instantaneously replaced by diffusion. In order to avoid bleaching bias, it is nevertheless 
recommended to use a low excitation power to avoid saturation, to use averaging, and to replace the sample frequently.

- some optically sectioning imaging systems have cross-talk in their system. In such a case an axial image stack into a fluorescent solution can once serve as a proof for the cross-talk. However, it is not possible to calculate an eSIPchart. In these systems, e.g. line scanning confocal microscopes and spinning disk confocal microscopes, eSIPcharts can only be produced by scanning a thin uniform fluorescent layer.

- in case of severe refractive index mismatch, e.g. if a water solution is imaged with an oil immersion objective, the axial intensity distribution cannot be modelled with the Eqs. 11 to 17. As a matter of fact, no microscope system should be run under such imaging conditions.

\subsection{3 eSIPcharts and Complementary Calibration}

It has been shown that eSIPchart analysis is a versatile and powerful tool for the assessment of the characteristics of optically sectioning microscopes. Naturally, it competes with a set of different techniques. As far as field-dependent variations in optically sectioning fluorescence microscopy are concerned, the creation of an eSIPchart with a thin uniform fluorescent layer or a fluorescent solution is unchallenged. A classical test for illumination and resolution used to be the axial imaging of a mirror slide. This does not work in the fluorescence configuration since today's fluorescence filters block excitation light reliably. Field-dependent variations are often caused by the dichroic mirror, so removing the fluorescence filter is not an option for the calibration and optimisation of a certain channel. Additionally, by imaging a mirror slide, one neglects the effects of STOKES-shift and introduces refractive index mismatch. For these reasons, the main application for mirror slides is the check whether the axial resolution in non-fluorescent channels meets the principal expectations.

Another classical test for field-dependent intensity variations are fluorescent plastic slides. These are very bright and very photostable. But they have some important disadvantages: first, the refractive index of plastic matches neither the refractive index of water nor of oil, so strong spherical aberrations can be expected if the optical section shall be obtained from within the slide. Second, some high-quality water immersion objectives require the use of a cover slip. Since this needs to be attached to the fluorescent plastic slab and some 'embedding medium' needs to be added, the light must pass through many refractive elements before finally hitting the fluorescent plastic. Therefore, some of the observed 
field-dependent effects will be caused by the sample rather than by the microscope itself. Fluorescent plastics' main field of application is the delivery of a strong fluorescence signal, e.g. if the emission beam path needs to be aligned. Certainly, a standardised emission light source is the better option to achieve this (Cho and Lockett, 2006).

The general standard for the evaluation of microscope performance is the recording and averaging of subresolution fluorescent beads to obtain the microscope's PSF. This yields information about the lateral and axial resolution of a fluorescence microscope, but it can neither give information concerning the optical sectioning strength nor field-dependent variations. Fluorescent beads are at present the only calibration standard with which it is possible i) to record the wavelength-dependent lateral resolution, ii) to laterally align a multichannel fluorescence microscope for chromatic aberrations, iii) to give the input parameters for deconvolution. Hence, they complement the eSIPchart analysis in a perfect manner. Nevertheless, standardised gridded cover slips might eventually enable the eSIPchart-approach to enter this domain of fluorescent beads. Then, the unique feature of subresolution fluorescent beads for calibration will be the use for empirical deconvolution.

To put the whole matter into a nutshell, it is recommended to perform a PSF-recording and a solution eSIPchart analysis on a weekly basis for heavy-duty optically sectioning fluorescence microscopes. It will be possible to develop a complete comprehension of the microscope system only if both measures are taken, and only then it is possible to optimise the system for a given imaging task. Table 1 shows a comparison of the complementary determinable microscope parameters.

Table 1: Complementary determinable microscope parameters. In addition to that, solution eSIPchart analyis is better suited for optimising a correction collar for a given imaging task.

\begin{tabular}{cc}
\hline PSF & eSIPchart analysis \\
\hline lateral resolution & field-dependent variations \\
lateral alignment & axial alignment \\
axial resolution & optical sectioning strength \\
principal spherical aberrations & field-dependent spherical aberrations \\
deconvolution & correction collar optimisation \\
\hline
\end{tabular}




\subsection{Exemplary eSIPcharts of Some Standard Sectioning Microscopes}

The following pages show exemplary eSIPcharts obtained on a ZEISS LSM510 (Fig. 30), an Olympus Fluoview 1000 (Fig. 26), a Zeiss LSM710 (Fig. 27), an Andor Revolution Spinning Disc confocal system (Fig. 28), and a LeicA TCS/SP2-system (Fig. 29). All microscopes are high end-instruments.

The ZEISs LSM710 and the OLYMPUs FLuOVIEW 1000 are direct competitors on the market, and they exhibit indeed a comparable performance. The FLUOVIEW 1000 is better aligned, which is no surprise, since this was a fixed installation in a laboratory, whereas the LSM710 was presented on a trade show. Note that the scan area in the FLUOVIEW 1000 is bigger, and that the intensity in the periphery drops accordingly. Both systems would profit from a beam expander in order to illuminate the back aperture more homogeneously. The FLUOVIEW 1000 has somewhat more spherical aberration than the LSM710.

Especially if speed matters, spinning disc or line scanning confocal systems are the imaging method of choice. I assessed an ANDOR REVOLUTION spinning disc confocal system on an exhibition. Set up for optimal resolution conditions, the system has somewhat worse axial resolution than the aforementioned ones. Unlike the Fluoview 1000 and the LSM710, the REVOLUTION spinning disc shows an artefact circular stripe pattern which stems from the circulating disc and might be related to insufficient dampening of the table. The field of view is very small for a confocal system. The rectangular shape of the field of view corresponds to the dimensions of the CCD-chip that is used for imaging.

The LEICA TCS/SP2-system is a direct competitor of the ZEISS LSM510META and was assessed on a fixed laboratory system. The alignment appears to be considerably good, and the resolution is the best that could be determined. Indeed, the illumination of the back aperture appears to be more homogeneous than in other systems. The trade off is signal intensity, which made a large binning kernel $(8 \times 8$ pixels $)$ necessary. Additionally, the intensity map shows a severe circular and linear stripe-artefact pattern that has no equivalent in the other maps. The reason for that pattern might be attributed to some sort of electronic feedback. There is relatively much spherical aberration in the system.

An exemplary eSIPchart recorded using fluorescent solution is shown in Fig. 30. The intensity distribution is almost perfect, but the angled positioning of the sample somewhat hinders the precise recording of the additional parameters. It can nicely be seen that axial position, weight factor and resolution correlate. The addition of a parameter in the fit model leads to a very narrow fit of the model function to the data, which is revealed by the $\chi_{\mathrm{R}}^{2}$-map. 

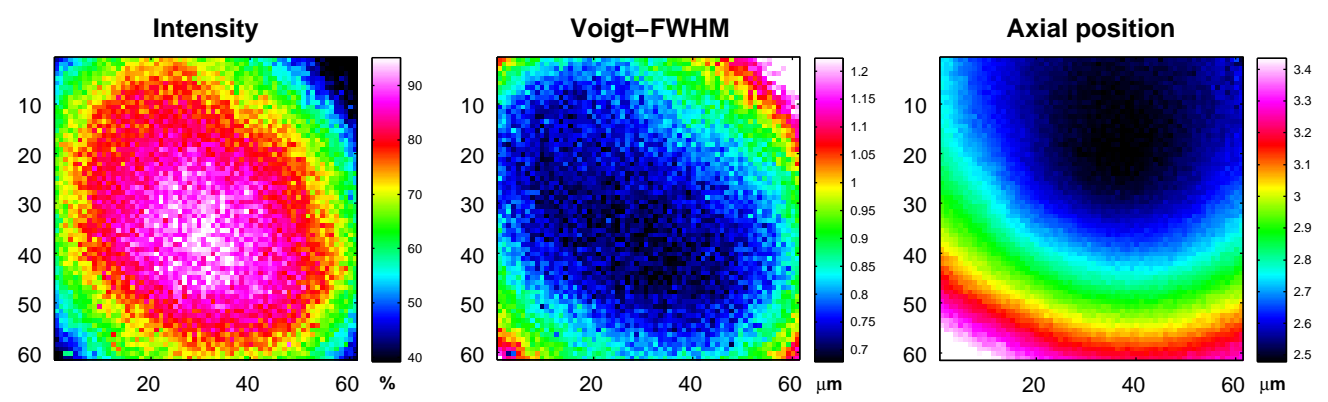

\section{eSIPchart}

\section{FLUOVIEW FV1000}

\section{Objective: 60/1.35 UPLSAPO}

$\lambda_{\text {ex }}=488 \mathrm{~nm}, \lambda_{\text {em }}=510 \mathrm{~nm}$

Field of view: $212 \times 212 \mu \mathrm{m}^{2}$, bin $4 \times 4$ from $256 \times 256$ Pixel.

08.12 .2008

\begin{tabular}{|llll|}
\hline & Average & \multicolumn{1}{c}{ SD } & Unit \\
\hline$I_{\max }$ & 43 & \pm 7.2 & Photons \\
\hline FWHM & 0.81 & \pm 0.11 & $\mu \mathrm{m}$ \\
\hline$z_{0}$ & 2.8 & \pm 0.25 & $\mu \mathrm{m}$ \\
\hline Offset & $1.8 \mathrm{e}+003$ & \pm 82 & $\mathrm{ADU}$ \\
\hline Skew & -0.0025 & \pm 0.0033 & $1 / \mu \mathrm{m}$ \\
\hline$\chi_{\mathrm{R}}^{2}$ & 0.76 & \pm 0.22 & \\
\hline
\end{tabular}
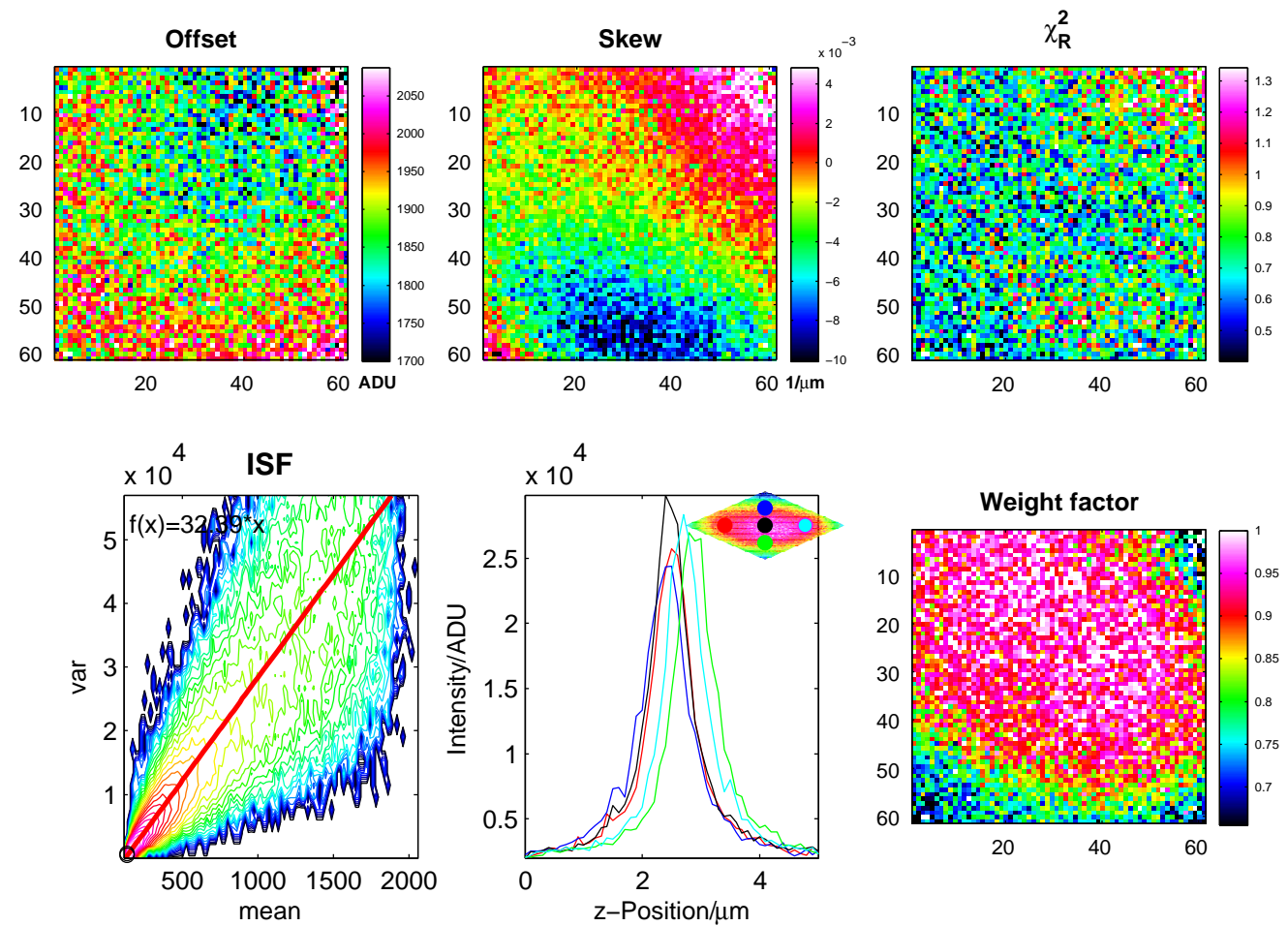

Figure 26: eSIPchart made on an Olympus Fluoview 1000. Scan of a layer. This system is almost perfectly calibrated. The relatively low photon number is sufficient to extract the parameters of interest. The weight-factor map reveals that the - good axial resolution could still be improved by using a beam expander. The system shows considerable spherical aberrations. 

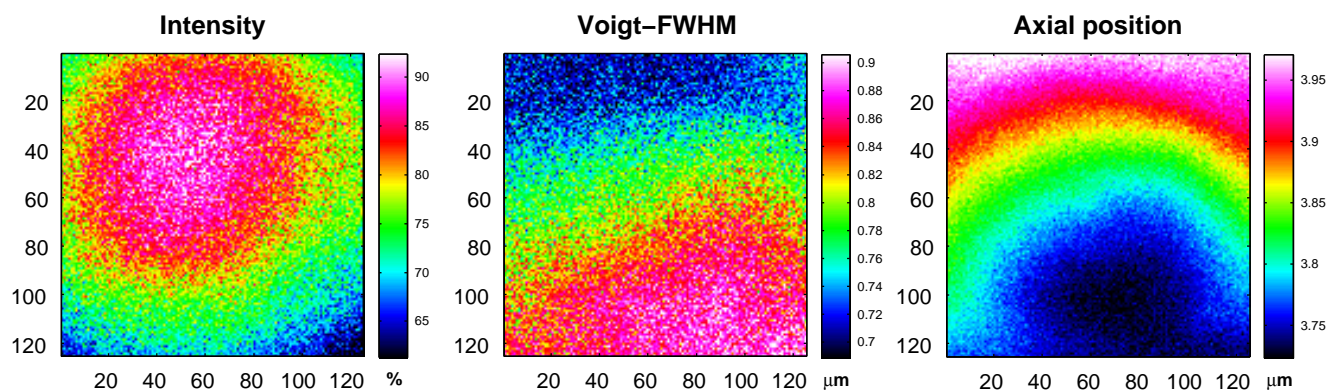

\section{eSIPchart}

\section{LSM710}

Objective: 63/1.4 Plan-Apochromat $\lambda_{\text {ex }}=488 \mathrm{~nm}, \lambda_{\text {em }}=536.0376 \mathrm{~nm}$

Field of view: $175 \times 175 \mu \mathrm{m}^{2}$, bin $4 \times 4$ from $512 \times 512$ Pixel.

07.12 .2008

\begin{tabular}{|llll|}
\hline & Average & \multicolumn{1}{c}{ SD } & Unit \\
\hline$I_{\max }$ & 42 & \pm 3.9 & Photons \\
\hline FWHM & 0.8 & \pm 0.058 & $\mu \mathrm{m}$ \\
\hline $\mathrm{z}_{0}$ & 3.8 & \pm 0.073 & $\mu \mathrm{m}$ \\
\hline Offset & $5.9 \mathrm{e}+003$ & $\pm 8.1 \mathrm{e}+002$ & $\mathrm{ADU}$ \\
\hline Skew & 0.0023 & \pm 0.0012 & $1 / \mu \mathrm{m}$ \\
\hline$\chi_{\mathrm{R}}^{2}$ & 1.4 & \pm 0.28 & \\
\hline
\end{tabular}
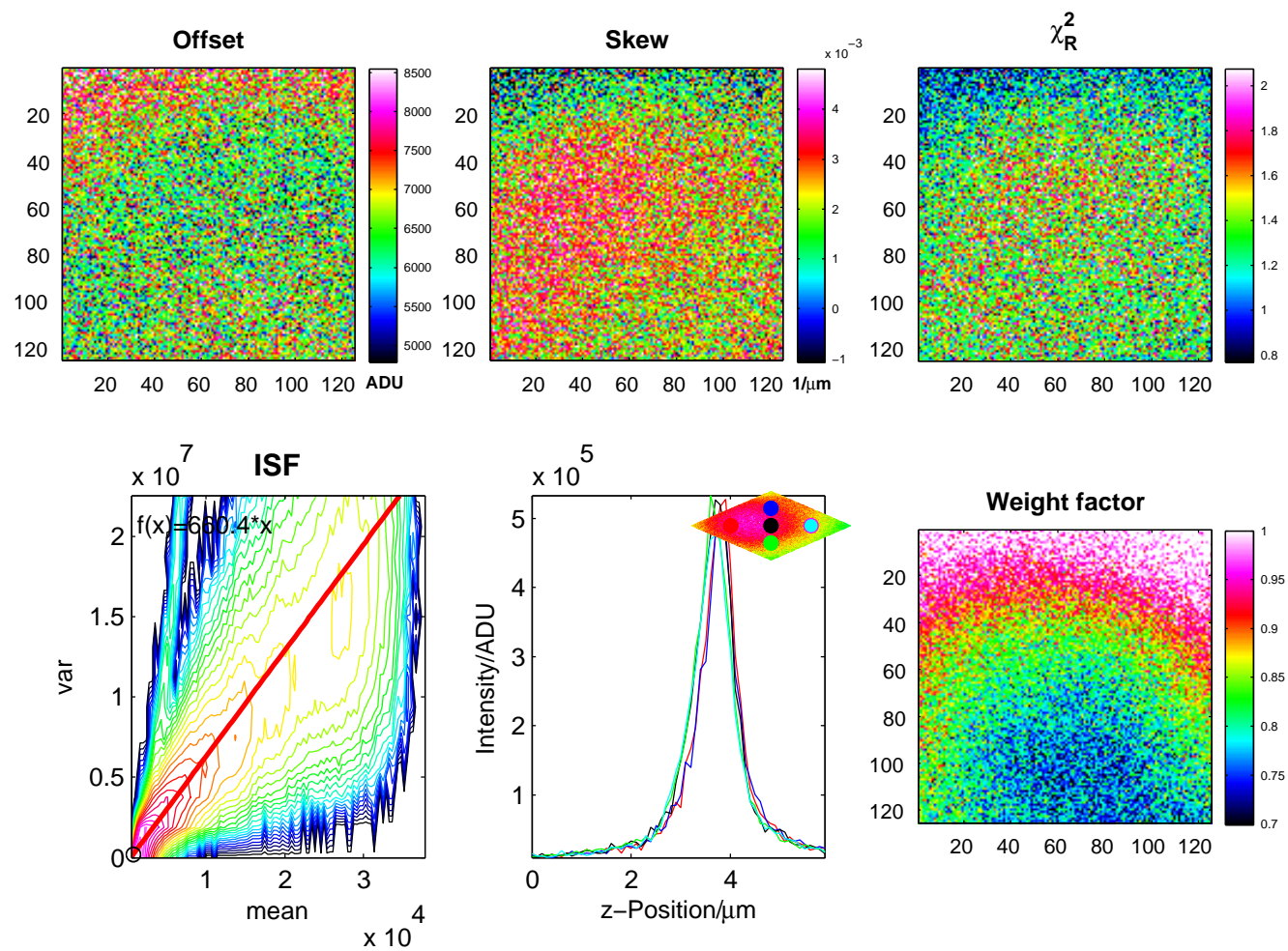

Figure 27: eSIPchart made on a ZeISs LSM710. Scan of a layer. Considering the absolute values, the LSM710 investigated here performs similarly to the OLYMPUS FLuOviEW 1000. The major difference is a shifted resolution map and an inverted weight factor map. Like in the FLUOVIEW 1000, a beam expander could improve the resolution. It has to be mentioned that the LSM710 was an exhibition system, whereas the Fluoview 1000 was a heavy duty laboratory system. 

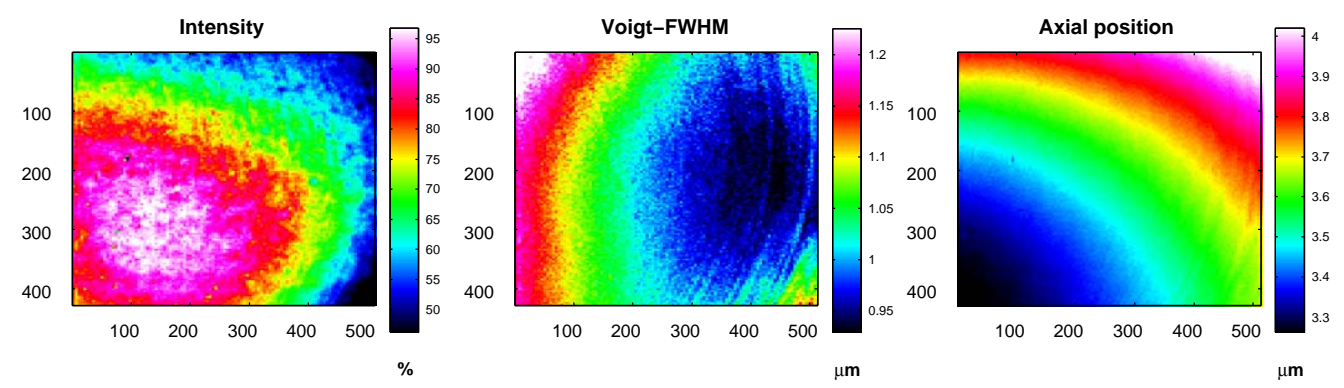

\section{eSIPchart}

\section{Andor REVOLUTION Sp.-Disc}

Objective: $60 \mathrm{x} / 1.45$ Oil

$\lambda_{\mathrm{ex}}=488 \mathrm{~nm}, \lambda_{\mathrm{em}}=525 \mathrm{~nm}$

Field of view: $114 \times 96 \mu \mathrm{m}^{2}$, bin $4 \times 4$ from $512 \times 431$ Pixel

27.03.2008

\begin{tabular}{|llll|}
\hline & Average & \multicolumn{1}{c}{ SD } & Unit \\
\hline$I_{\max }$ & $3.2 \mathrm{e}+002$ & \pm 59 & Photons \\
\hline FWHM & 1 & \pm 0.075 & $\mu \mathrm{m}$ \\
\hline $\mathrm{z}_{0}$ & 3.6 & \pm 0.18 & $\mu \mathrm{m}$ \\
\hline Offset & $9.3 \mathrm{e}+003$ & $\pm 1.4 \mathrm{e}+003$ & counts \\
Skew & 0.0029 & \pm 0.0087 & $1 / \mu \mathrm{m}$ \\
\hline$\chi_{\mathrm{R}}^{2}$ & 2.9 & \pm 1 & \\
\hline
\end{tabular}
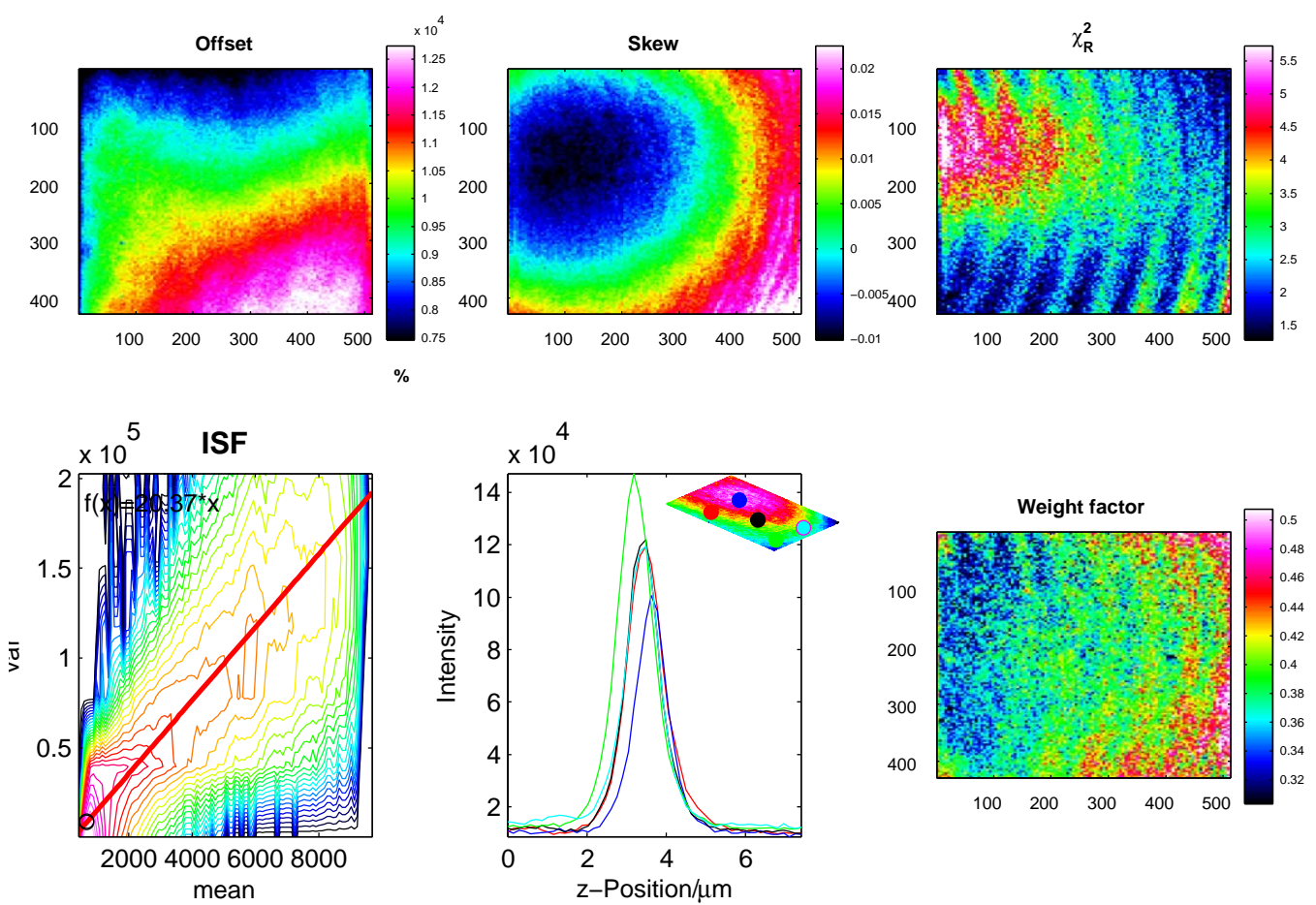

Figure 28: eSIPchart made on a demonstration Andor Revolution Spinning Disc confocal system. Scan of a layer. As mentioned previously, a spinning disc system exhibits too much crosstalk to be investigated with a fluorescent solution. The system under investigation not unexpectedly shows circular stripe-artefacts that might arise from vibrations. Interestingly, these are not visible in the intensity map. The axial resolution is somewhat worse than in single point scanning confocal systems. 

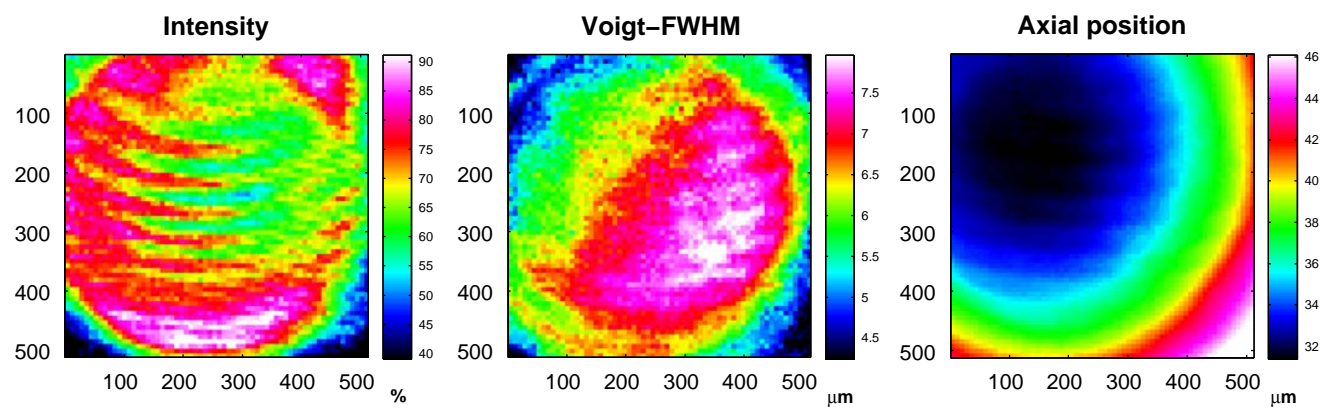

\section{eSIPchart}

\section{Leica TCS/SP2}

Objective: HCX PL APO CS 63x1.4 Oil

$$
\lambda_{\text {ex }}=488 \mathrm{~nm}, \lambda_{\text {em }}=505 \mathrm{~nm}
$$

Field of view: $512 \times 512 \mu \mathrm{m}^{2}$, bin $8 \times 8$ from $512 \times 512$ Pixel.

\begin{tabular}{|llll|}
\hline & Average & \multicolumn{1}{c|}{ SD } & Unit \\
\hline$I_{\max }$ & 13 & \pm 1.9 & Photons \\
\hline FWHM & 6.4 & \pm 0.89 & $\mu \mathrm{m}$ \\
\hline$z_{0}$ & 36 & \pm 3.7 & $\mu \mathrm{m}$ \\
\hline Offset & $8.1 \mathrm{e}+003$ & $\pm 1.2 \mathrm{e}+002$ & counts \\
\hline Skew & -0.026 & \pm 0.0063 & $1 / \mu \mathrm{m}$ \\
\hline$\chi_{\mathrm{R}}^{2}$ & 0.46 & \pm 0.24 & \\
\hline
\end{tabular}

26.01.2009
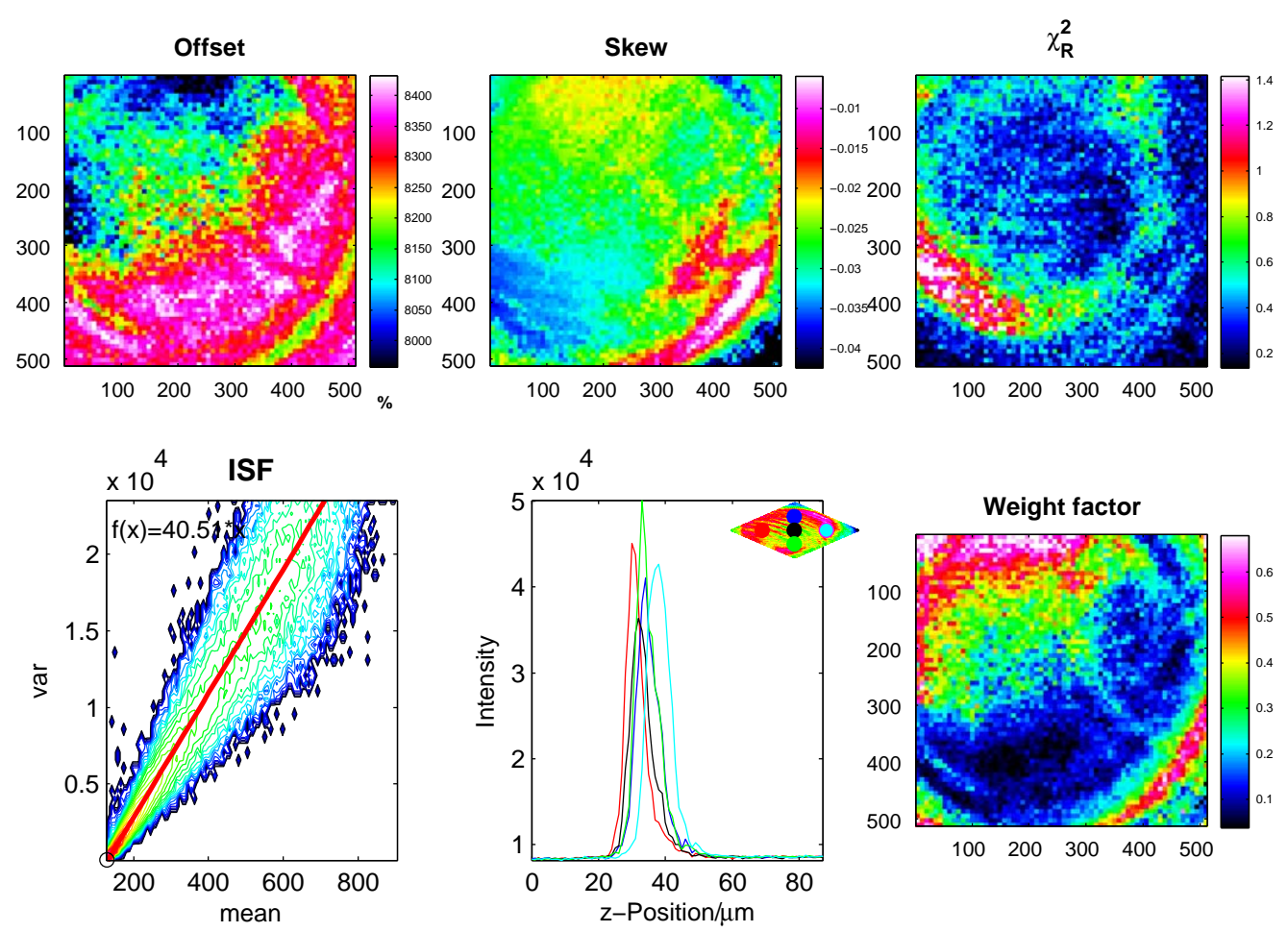

Figure 29: eSIPchart made on a LEICA TCS/SP2 confocal system. Scan of a layer. From all systems under investigation, this system has the best axial resolution. The reason for this is a comparably expanded excitation beam. Nevertheless, there is an unspecified stripe-artefact in the intensity map and a dominant ring-structure on all maps. Due to the low photon number, a relatively large binning kernel of $8 \times 8$ pixels had to be used, which renders the determination of the true gain somewhat less reliable (cf. section 2.4). 

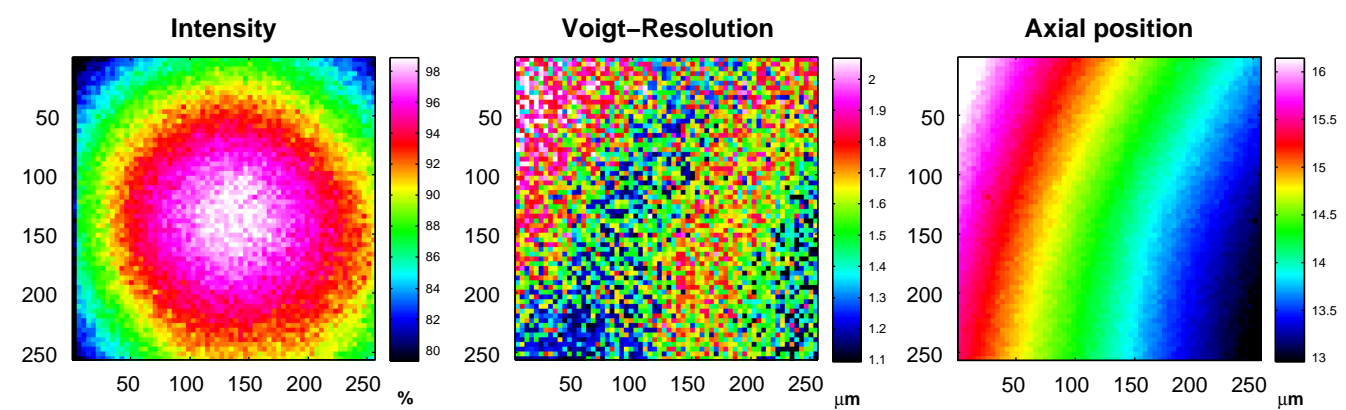

\section{eSIPchart}

\section{Zeiss LSM510 META PH1}

Objective: C-Apochromat 40x/1.2 corr

$\lambda_{\text {ex }}=488 \mathrm{~nm}, \lambda_{\mathrm{em}}=524 \mathrm{~nm}$

Field of view: $230 \times 230 \mu \mathrm{m}^{2}$, bin $4 \times 4$ from $256 \times 256$ Pixel.

20.09.2008

\begin{tabular}{|llll|}
\hline & Average & \multicolumn{1}{c}{ SD } & \multicolumn{1}{c|}{ Unit } \\
\hline$I_{\max }$ & $2.2 \mathrm{e}+002$ & \pm 11 & Photons \\
\hline FWHM & 1.6 & \pm 0.23 & $\mu \mathrm{m}$ \\
\hline$z_{0}$ & 14 & \pm 0.82 & $\mu \mathrm{m}$ \\
\hline Offset & $3.3 \mathrm{e}+003$ & \pm 42 & counts \\
\hline $\mathrm{k}$ & 0.0017 & \pm 0.00014 & $1 / \mu \mathrm{m}$ \\
\hline$\chi_{\mathrm{R}}^{2}$ & 1 & \pm 0.17 & \\
\hline
\end{tabular}
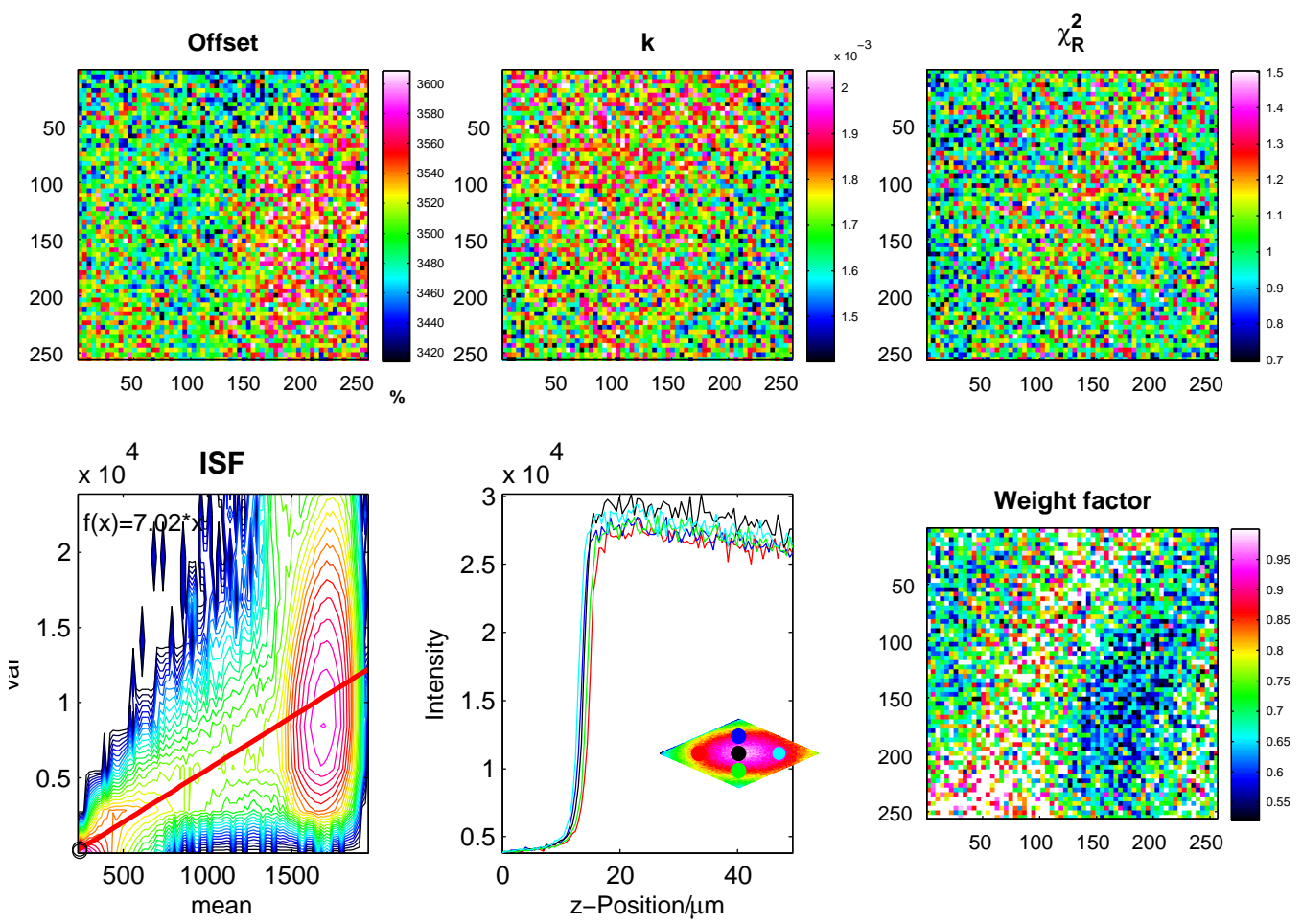

Figure 30: eSIPchart made on a ZEISS LSM510. Scan in solution. The intensity distribution and the axial position are robustly determined. The resolution map and the weight factor map shows a distribution that correlates with the relatively angled positioning of the probe on the microscope table. The $\chi_{\mathrm{R}}^{2}$ map shows that the data can be fitted well. In addition, the calculation of the gain factor is considerably reliable. 


\section{Quantitation in Structured Illumination Microscopy}

Structured illumination microscopy (SIM) is a new approach in fluorescence microscopy with the aim to obtain optical sections from widefield-epifluorescence images. Compared to a standard point-scanning cLSM it is easier to use, spectrally more versatile, and cheaper. Depending on the implementation it can be faster and it can offer a better resolution. Despite this commercial realisations of this technique are rare.

During working on this part of the thesis, physical and biological tests were developed for the evaluation of microscope performance. The ultimate goal was to give users in biology sufficient information at hand to decide in which cases SIM is an alternative to established fluorescence microscopy techniques.

This section is divided into three parts: after a brief introduction into the basic principles of structured illumination microscopy and the commercial representation used here, I will present in detail the results I obtained from comparative studies. Finally, I will discuss potentials and pitfalls of structured illumination microscopy and suggest possible improvements.

\subsection{Principles of Linear Grid Structured Illu- mination}

\subsubsection{Confocal vs. Widefield Epifluorescence Microscopy}

The main advantage of confocal microscopy compared with widefield epifluorescence microscopy is its capability of obtaining optical sections from the sample. Optical sections have two major advantages: i) an axial image stack of optical sections taken at a sufficiently small distance which fulfils the demands of the Nyquist-theorem (Nyquist, 1924, 1928) in the best case allows a 3D-reconstruction of the sample. In doing so it is not only possible to visualise properly the sample as a structure that extends in $3 \mathrm{D}$, but also to perform colocalisation experiments that are justified based on the confinement of the detection volume. ii) out-of-focus light that seriously degrades images in widefield-epifluorescence microscopy is rejected by the confocal pinhole. These advantages are bought by some serious 
costs which are presented in the following. First of all, laser illumination is needed. Only lasers provide the intensity required along with the well-defined diffraction limited spot in focus to scan a sample sufficiently fast. Second, only available laser wavelengths can be used. Arc lamps are usually used as excitation source in widefield epifluorescence microscopy. This is why it is possible to select the excitation from $300-1000 \mathrm{~nm}$, depending only on the objective's transmission characteristics, whereas it is either impossible or very expensive (tunable lasers, UV-lasers, frequency doubling) to use certain wavelengths in cLSM. The reason is that the excitation wavelength depends on the laser transitions available. The third point mentioned here is acquisition speed. As the sample has to be scanned, e.g. $1024 \times 1024$ pixels are scanned serially, while an image of the same size and resolution is recorded at one point in time using a common Megapixel CCD-camera. Naturally, the scan area can be smaller, so imaging would be faster. If an image of the same size and resolution is scanned for the same time with the demand to collect a similar amount of fluorescence photons, the laser intensity has to be turned up to values at which photodamage is likely to occur. Forth, alignment is laborious especially of the confocal pinhole. While most alignment steps in today's cLSM-units are performed automatically, the pinhole has to be aligned properly for every objective and every beam path configuration in use. The last point made here are the financial costs: prices for common laser-scanning units vary with the optional equipment and start at $\approx \mathrm{k} € 100$. Widefield imaging in contrast requires a CCD camera, a PC and some software, i.e. $\approx \mathrm{k} € 10$ to start with.

Taking all this into account, it would be convenient to have a widefield epifluorescence microscopy technique at hand that is capable of producing optical sections. Two techniques are available at present, both relying on a mathematical extraction of the in-focus image: deconvolution and structured illumination. Deconvolution requires an exact knowledge about the imaging system, particularly about its optical transfer function $(\mathrm{OTF})$. In short: as an image represents the object convolved with the microscope's point spread function (PSF), a deconvolution of the image with the OTF restores the image:

$$
I=O \otimes \mathrm{PSF} \rightsquigarrow \mathcal{F}\{O\}=\frac{\mathcal{F}\{I\}}{\mathrm{OTF}}
$$

$I$, image; $O$, object; $\otimes$, convolution operation; $\mathcal{F}$, Fourier transform. In this naive formulation, the algorithm is highly sensitive to noise. Notwithstanding many algorithms have been created which deal adequately with the noise (e.g. Pankajakshan et al., 2007; Schaefer et al., 2001). It is even stated that properly applied deconvolution improves the image quality not only of widefield microscopy, but also of all kinds of optically sectioning microscopy (Pawley, 2006). Structured illumination combines optical and mathematical elements. To be more precise, the 
microscope's field stop plane is manipulated to generate structured illumination on the object. The illumination structure serves as a label of the focal plane, as it rapidly attenuates with defocus. A set of images with shifted illumination structures guarantees that all parts of the object plane are illuminated at least once. Depending on illumination structure and employed algorithms, the in-focus image is calculated.

\subsubsection{Structured Illumination Microscopy}

Already in 1963, W. LukOsz inserted grids into the microscope's field stop plane and into the conjugated intermediate image plane to produce structured illumination. Fast conjugated lateral movement of the grids and imaging upon a temporally sufficiently inert detector enabled an improvement in lateral resolution (Lukosz and Marchand, 1963). The idea of using structured illumination to generate optical sections was first published by Neil et al., 1997. Again, a grid with a known grid constant is inserted into the field stop plane. Thus the object is illuminated by the grid pattern. The lateral intensity distribution $I(x, y)$ of a sinusoidal grid pattern in the focal plane is of the following form:

$$
I(x, y)=I_{0}+I_{\mathrm{s}} \cos \left(2 \pi \nu x_{0}+\phi_{0}\right)
$$

$I_{0}$ conventional wide-field image; $I_{\mathrm{s}}$ optically sectioned image; $\nu$ grid frequency; $\phi_{0}$ arbitrary phase. In shifting the grid at least twice by angles $\Delta \phi=\frac{\pi}{4}$ or $\Delta \phi=\frac{2}{3} \pi$ (Lagerholm et al., 2003), one assures a light exposure to the whole focal plane and minimises severe artefacts caused by the second harmonic grid frequency. In order to obtain the focal optically sectioned image, an elementary algebraic algorithm has to be carried out on a pixel-by-pixel basis: for $\Delta \phi=\frac{2}{3} \pi$,

$$
I_{\mathrm{s}}=\frac{\sqrt{2}}{3} \sqrt{\left(I_{1}-I_{2}\right)^{2}+\left(I_{2}-I_{3}\right)^{2}+\left(I_{3}-I_{1}\right)^{2}}
$$

The conventional image is recovered by:

$$
I_{\text {con }}=\frac{1}{3} \sum_{i=1}^{3} I_{i} .
$$

Fig. 31a shows a schematic representation of the underlying principle. The two grid position panels display two axial cuts through the object plane at two different grid positions and demonstrate how a grid structure is imaged axially into the object plane. In-focus structures are superposed by the grid pattern (red and green sphere), and therefore excited only in one or the other image. As a consequence they are only visible in a subset of images. Out-of-focus structures appear blurred in both images (blue sphere), because the high spatial frequency of the grid attenuates 

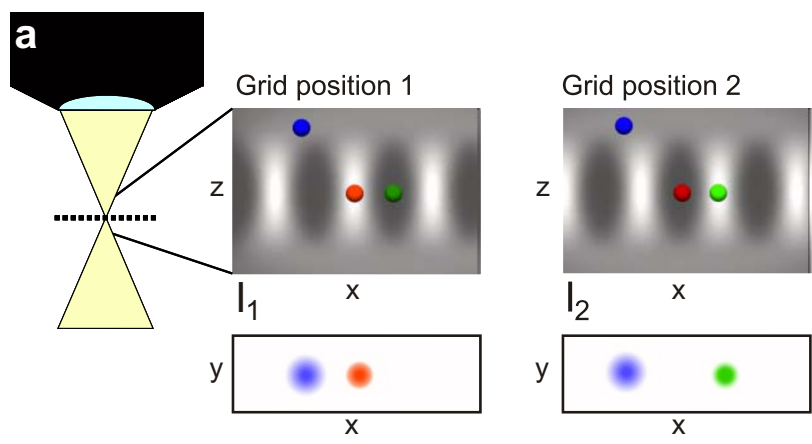

b
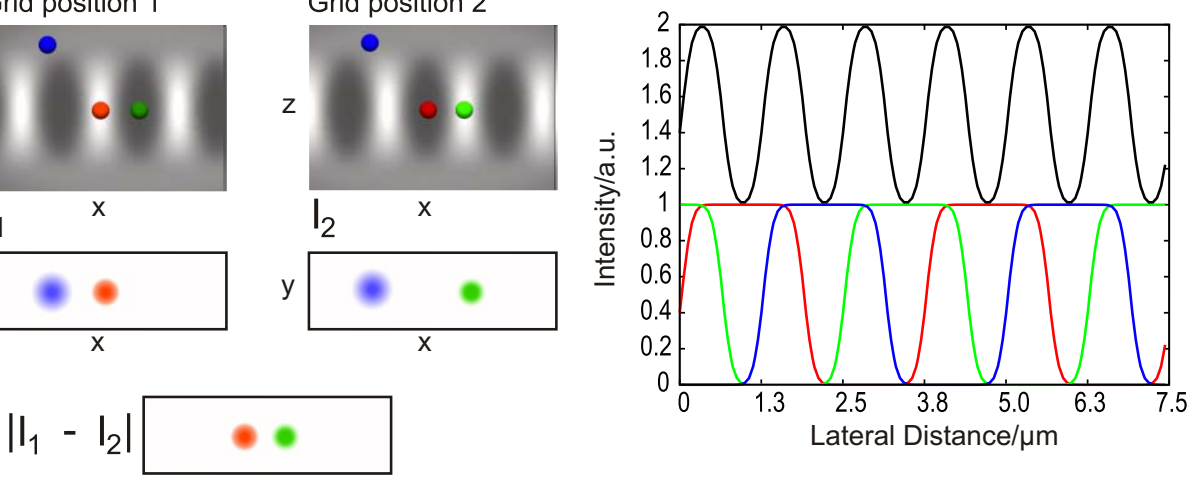

Figure 31: a) The simplified representation of the linear grid structured illumination principle shows how optically sectioned images are obtained. b) Red, green, and blue curve: grid pattern as it is imaged into the specimen for the calculation of one sectioned image. Black curve: summed intensity which the specimen is exposed to during one image acquisition.

with defocus. When the two images are subtracted from each other $\left(\left|I_{1}-I_{2}\right|\right)$, the out-of-focus contribution (blue sphere) will vanish, since it is present in both images. Only in-focus structures (red and green sphere) will contribute to the calculated optically sectioned image. In the actual ApoTome-algorithm, three images are taken (cf. Eq. 23) and alternately subtracted to exclude artefacts from the edges of the grid. Fig. 31b reveals a notorious problem of the raw image acquisition as it is done in the ApoTome. The summed illumination intensity for the acquisition of one optically sectioned image is inhomogeneous: it features an illumination structure that has three times the grid frequency. A result might be pattern bleaching. This particular implementation of SIM has been made commercially available by the ZEISS ApoTome and the QIOPTIQ OptiGrid. In both cases, the SIM module consists of an inset (grid slider) into the field stop plane along with sophisticated image reconstruction software that are added to a conventional widefield epifluorescence microscope. It follows from this that these commercial SIM implementations employ the standard excitation and detection hardware which was designed for widefield microscopy. It usually consists of arc lamp illumination and CCD-camera detection (see Fig. 33). The achievable sectioning strength in these systems depends on the available grid frequencies, which is rather inflexible with the two grids supplied. Furthermore, the light intensity generated by the arc lamp might be insufficient for fast imaging since three raw images have to be acquired to obtain one processed i.e. optically sectioned image. For these reasons, 
other strategies for illumination and image reconstruction have been developed:

- As a cure against the low excitation light intensity, laser illumination was used by Neil et al., 2000, which of course trades the low costs of arc lamp illumination systems. An additional benefit can be taken from the polarisation of the laser that yields optimal in-focus grid contrast if oriented parallel to the grid (Pawley, 2006).

- Lasers can also be used to create interference patterns which serve as a structured illumination pattern. The advantage of this approach is a wide range of possible grid frequencies, the disadvantage is a high sensitivity to external disturbance (Neil et al., 1998).

- If the grid is not only shifted but also rotated, it is possible to extend the spatial frequency space by making use of Moiré-fringes; spatial resolution (laterally and axially) can be improved by a factor of 2 (Gustafsson, 2000). In the $\mathrm{I}^{5} \mathrm{M}$-configuration this technique has been shown to yield an isotropic resolution of $100 \mathrm{~nm}$ (Shao et al., 2008). ${ }^{5} \mathrm{M}$ stands for a microscopy technique in which two opposing lenses are widefield illuminated, and the interference of the opposing beams reveals high resolution information (Gustafsson et al., 1999). Since for one processed image many raw images are required (15 in the cited paper) and interference is sensitive to setup and sample movements, this technique trades robustness and temporal resolution for spatial resolution.

- In the programmable array microscope, a freely programmable digital micromirror device is used to generate arbitrary illumination patterns (Fulwyler et al., 2005; Hanley et al., 1999, 2000; Hanley et al., 2005; Heintzmann et al., 2001; Mitić et al., 2003). Since the fluorescence light also passes the digital micromirror device, the system generates optical sections online in an analogue way as Lukosz and Marchand, 1963 did with linear grid structured illumination and Petráň et al., 1968 did with the spinning disc.

All modes of structured illumination bear the feature in common that the illumination pattern needs to be efficiently imaged into the focal plane: the in-focus amplitude modulation encodes the information to be extracted by SIM-techniques and algorithms. If the illumination pattern is imaged into the specimen, it will be convolved with the microscope's PSF and therewith follow the classical laws of diffraction. In general, this effect will diminish with larger structures, that is with lower spatial frequencies of the illumination pattern, and smaller mark-area ratios.

Concerning grid projection, a grid pattern with a low spatial frequency and a mark-area ratio of $\frac{1}{2}$ will only slightly be smeared out at the edges of the single 


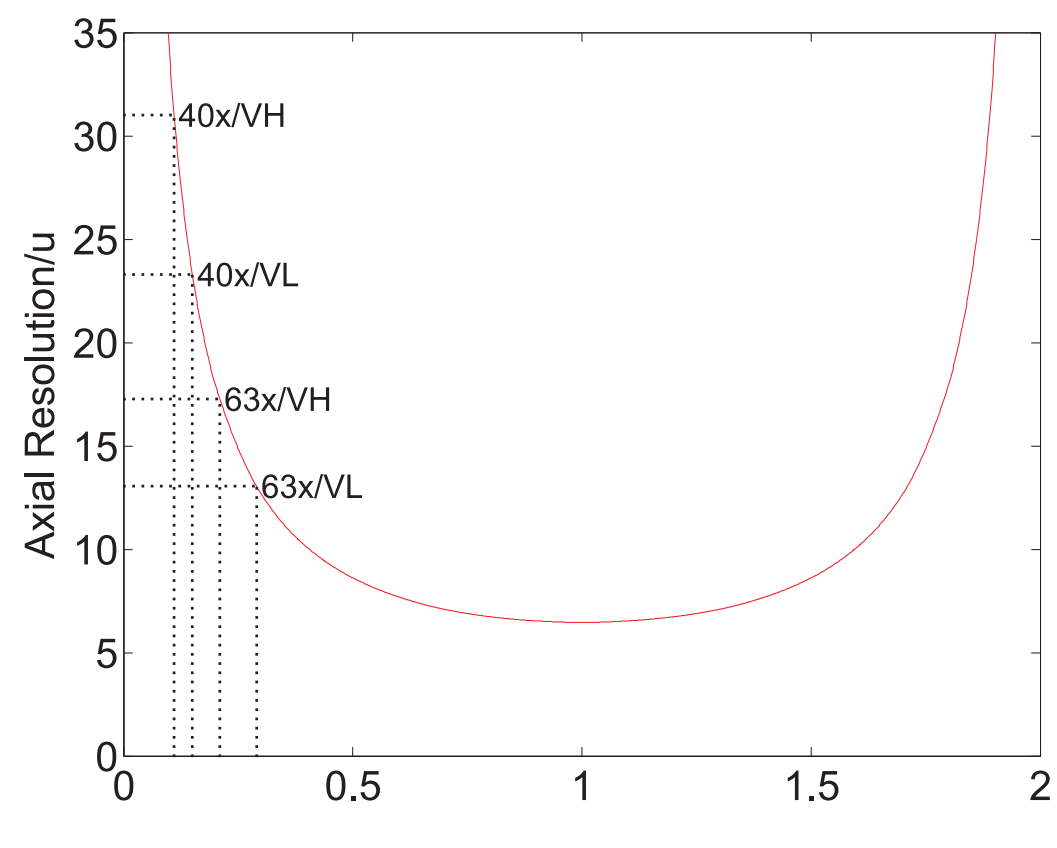

Figure 32: The axial sectioning strength as it depends on the normalised grid frequency $\tilde{\nu}$ (after Stokseth, 1969). Sectioning will be best for $\tilde{\nu}=1$. The position of the objective/grid combinations is also given. The STOKSETH-approximation is inappropriate for values $\tilde{\nu}<0.05$ and $\tilde{\nu}>1.95$.

lines, and the amplitude will remain unaffected, while a grid with a high spatial frequency will be severely demodulated. At a certain grid frequency, the modulation will completely vanish. This is exactly the way how E. ABBE's described the resolution limit in optical systems (Abbe, 1873). From this it becomes clear that the illumination optics plays an important role in SIM: the structure has to be imaged as good as possible into the specimen. Since this is not so important in standard widefield epifluorescence microscopy, the illumination optics in many standard microscopes is not corrected as good as possible. Therefore, the imaged structure suffers from well-known artefacts, like spherical and chromatic aberrations and field curvature, which all result in more or less severe artefacts in the processed image.

There is an upper (diffraction) limit to the imaging of the grid even in artefactfree systems, so the question arises of how fine the grid can be to yield optimal sectioning and resolution performance. This depends naturally upon the actual settings, i.e. the diffraction limit given by the actual objective in use. When denoting $\nu$ as grid frequency given in lines $/ \mathrm{mm}$, the normalised grid frequency will be $\tilde{\nu}=\frac{M \lambda \nu}{N A} ; M$, magnification between grid and object plane; $\lambda$, wavelength, $N A$, numerical aperture. Using optical coordinates, i.e. $u=4 k n z \sin ^{2} \frac{\alpha}{2}, k=\frac{2 \pi}{\lambda}$, with $n$, refractive index and $\alpha$, semi-angle of the beam spread, the axial intensity 
profile of a subresolution fluorescent layer can be approximated by (Neil et al., 1997; Stokseth, 1969):

$$
I_{\mathrm{s}}(u, \tilde{\nu}) \propto\left|\frac{2 J_{1}\left(u \tilde{\nu}\left(1-\frac{\tilde{\nu}}{2}\right)\right)}{\left(u \tilde{\nu}\left(1-\frac{\tilde{\nu}}{2}\right)\right)}\right|^{2}
$$

$J_{1}$, Bessel-function of the first order, first kind. Optimal sectioning strength and resolution can be obtained using $\tilde{\nu}=1$ (see Fig. 32). In practise, the low modulation depth given by out-of-focus components requires $\tilde{\nu} \leq 0.5$. Eventually, if $\tilde{\nu}=0$ is chosen, i.e. if the grid is removed, $I_{\mathrm{s}}=$ const., so all sectioning capability will have vanished.

\subsubsection{The ApoTome Setup}

Two microscope systems were used for the structured illumination experiments:

\begin{tabular}{lcc}
\hline & Configuration 1 & Configuration 2 \\
\hline Microscope body & Axiovert 200M & AxioObserver.Z1 \\
CCD camera & AxioCam HR & AxioCam MRm \\
Pixel number & $1300 \times 1030$ & $1388 \times 1040$ \\
Pixel size & $6.7 \mu \mathrm{m} \times 6.7 \mu \mathrm{m}$ & $6.45 \mu \mathrm{m} \times 6.45 \mu \mathrm{m}$ \\
Ex. arc lamp & xenon $(\mathrm{XBO} 75)$ & metal halide $(120 \mathrm{~W})$ \\
Software & AxioVision 4.4 & AxioVision 4.6 \\
\hline
\end{tabular}

All equipment was purchased from ZeISS (Jena, Germany), apart from the metal halide short arc lamp (EXFO, Mississauga, Canada). The same objectives and filter sets were employed on both setups: either a 40x/1.2W C-Apochromat or a $63 \mathrm{x} / 1.4$ Plan-Apochromat (oil) objective, and the ZEISS-filter sets 10, 49, $38 \mathrm{HE}, 43$, and 32 were used. For structured illumination measurements the ApoTome-slider was introduced into the field stop plane. Thereby one of two available rectangular-shaped gratings was inserted into the field stop plane, either a grid with $\nu_{\mathrm{VH}}=17.5$ lines $/ \mathrm{mm}$ or a grid with $\nu_{\mathrm{VH}}=35$ lines $/ \mathrm{mm}$. "VH" stands for the designated use in connection with high magnification objectives in an Axiovert microscope and "VL" stand for the designated use with low magnification objectives. The use in the newer AxioObserver.Z1 poses no problem though.

Table 2 shows the normalised grid frequencies employed here. It is quite obvious that the purpose of the ApoTome is not to obtain the best resolution possible, but to compromise between sectioning, noise, and recording time required. The recommended grid frequency $(\mathrm{VH})$ for the use with high-magnification-objectives is considerably below the one that achieves optimal resolution. Even if the (not 


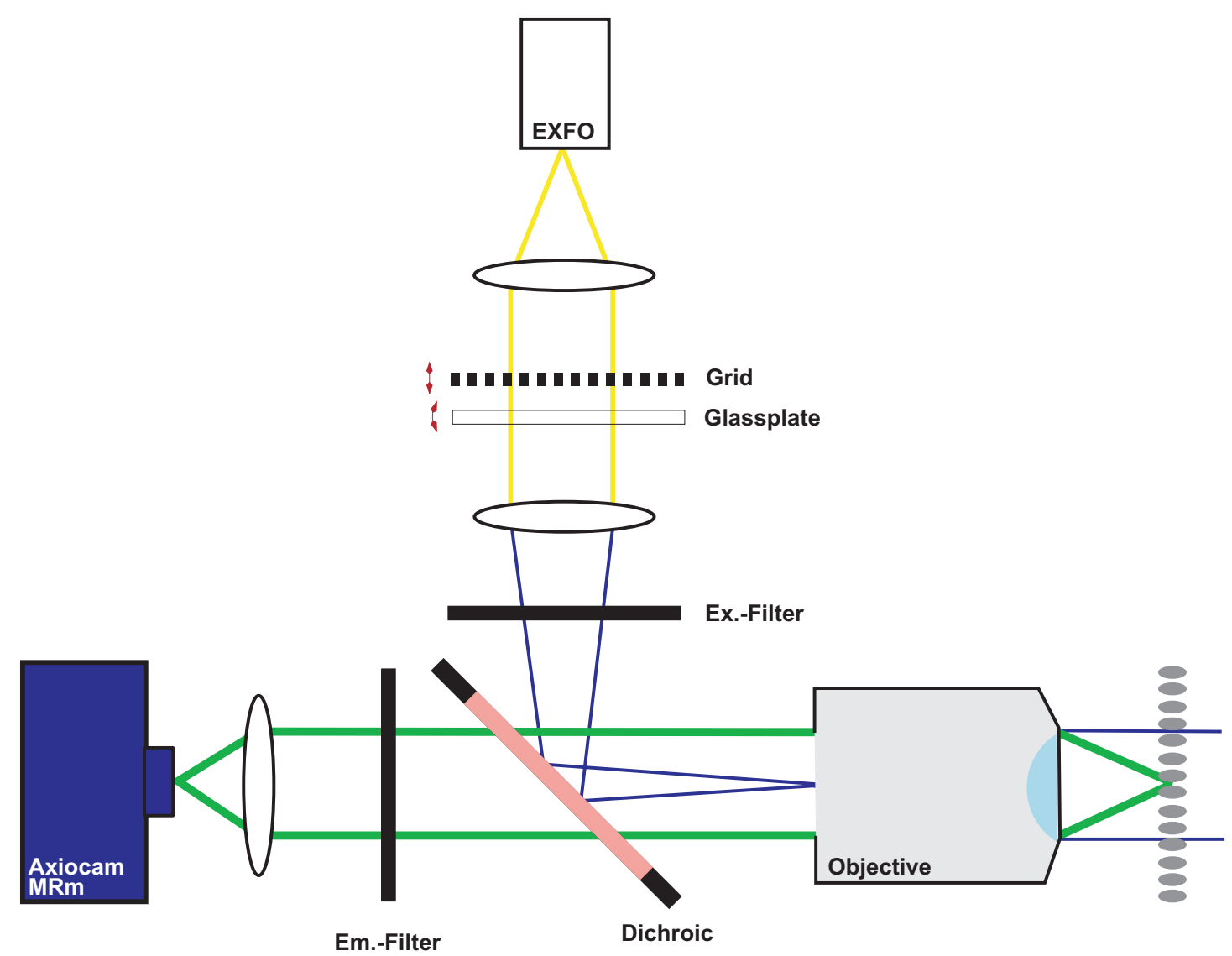

Figure 33: Image formation in the ApoTome: a grid is inserted into the object's conjugate image plane at the field stop and hence imaged onto the object. The grid is positioned according to the selected excitation wavelength. The glass plate operates as a phase shifter. The object is widefield-epi-illuminated three times with the grid image shifted by $\Delta \phi=\frac{2}{3} \pi$, and the fluorescence image is projected onto a CCD-chip.

recommended) high-frequency grid (VL) designated for the use with low magnification objectives is inserted, one is still far away from measuring with an optimum resolution. The highest normalised grid frequency on such a system could be obtained by using a 100x/1.4 oil immersion objective, which yields $\tilde{\nu}=0.49$.

Grid phase and focus calibration were performed prior to each experiment. The acquired image stacks were exported as 16 bit TIFF-images and processed using custom software written in Matlab.

\subsection{Characterisation of the ApoTome}

\subsubsection{Resolution}

The ApoTome's resolution performance was assessed by using fluorescent beads (Murray et al., 2007) and a thin homogeneous fluorescent layer (Zwier et al., 

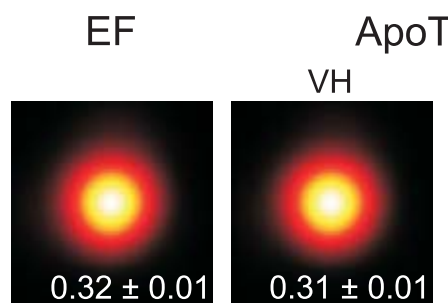

ApoTome

CLSM
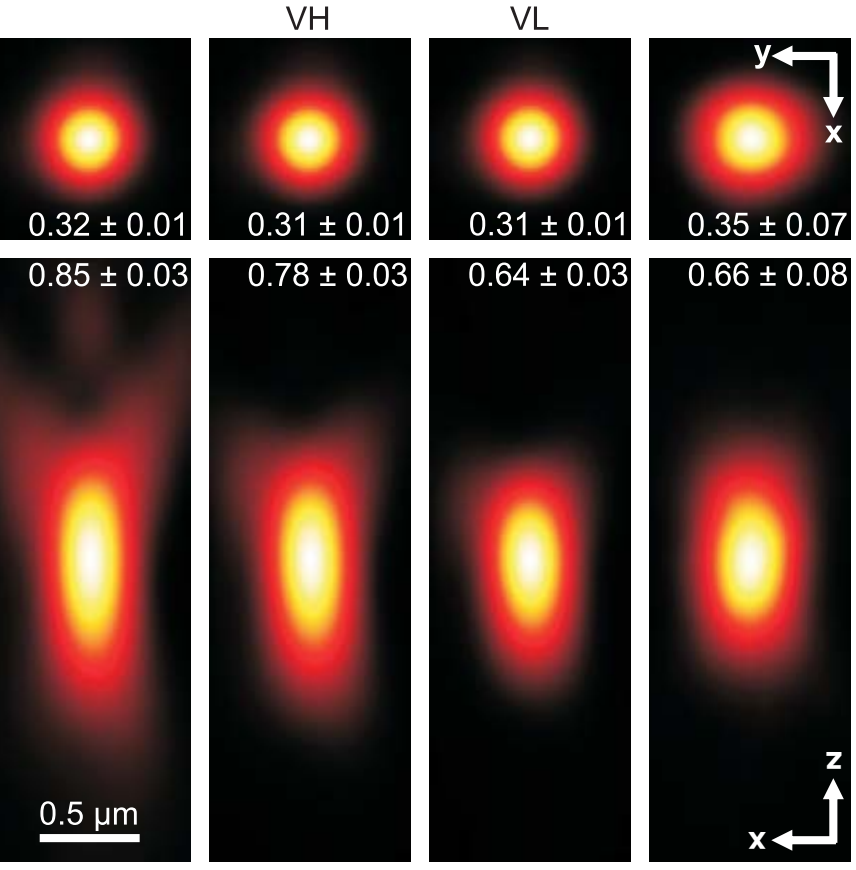

$0.64 \pm 0.03$

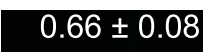

Figure 34: Lateral and axial PSFs as obtained from $175 \pm 5 \mathrm{~nm}$ green fluorescent beads. PSF images were produced by manually selecting $N \gtrsim 100$ beads from an image stack for every imaging mode, aligning them with sub-pixel precision to their centre of gravity and calculating the mean. The axial resolution in the cLSM, $d_{\mathrm{p}}=1 \mathrm{~A} . \mathrm{U}$. and in the ApoTome, VL-grid, are similar. VH, ApoTome low sectioning ( $\left.\tilde{\nu}_{\mathrm{VH}}=0.15\right)$; VL, ApoTome high sectioning $\left(\tilde{\nu}_{\mathrm{VL}}=0.29\right)$; EF, conventional epifluorescence; cLSM, confocal laser scanning microscope.

2004) as described before (section 2.2.1), and it was compared to the resolution performance of a cLSM 510 (ZEISS) and conventional epifluorescence microscopy. For a $z$-stack, 60 sectioned images at $100 \mathrm{~nm}$ step width were taken. To ensure optimal resolution conditions, the 63x/1.4 Plan-Apochromat-objective was used, and fluorescence objects were attached to the cover slip.

Table 2: Normalised grid frequencies in the ApoTome setup assuming an excitation wavelength of $\lambda=500 \mathrm{~nm}$, and relative axial resolution.

\begin{tabular}{ccccc}
\hline Objective & $\tilde{\nu}_{\mathrm{VH}}$ & $\tilde{\nu}_{\mathrm{VL}}$ & $d_{z, \mathrm{VH}, \mathrm{rel}}$ & $d_{z, \mathrm{VL}, \mathrm{rel}}$ \\
\hline $40 \mathrm{x} / 1.2$ & 0.11 & 0.21 & 0.21 & 0.37 \\
$63 \mathrm{x} / 1.4$ & 0.15 & 0.29 & 0.28 & 0.49 \\
\hline
\end{tabular}




\section{Lateral Resolution}

Radial and axial central sections of the extracted PSFs are shown in Fig. 34 for epifluorescence (left), for the ApoTome with VH-grid or VL-grid (middle), and for the cLSM (right). There is no significant difference between the ApoTome and the standard epifluorescence microscope with regard to lateral resolution, whereas the cLSM's lateral PSF is slightly broader. The resolution of the cLSM is determined by the size of the emission pinhole, and for maximally closed pinholes the confocal imaging theory predicts an improvement of $\sqrt{2}$ over epifluorescence (Wilson, 1990), with $95 \%$ of the in-focus-light being rejected by the pinhole (Sandison et al., 1995). A pinhole size of $d_{\mathrm{p}}=1 \mathrm{~A}$.U. is often considered a good compromise between resolution and detected light intensity (Pawley, 2006). If, in this case, the backfocal plane (BFP) is illuminated homogeneously, the lateral resolution of the cLSM should outperform epifluorescence by $\frac{\lambda_{\mathrm{ex}}}{\lambda_{\mathrm{em}}}$. In these measurements the cLSM's lateral resolution fell behind that of epifluorescence or the ApoTome: $d_{l, \mathrm{EF} / \mathrm{VH} / \mathrm{VL}} \cdot 1.1=d_{l, \mathrm{cLSM}}$. This may be explained, first, by the GaUsSian intensity profile in the BFP, which reduced the effective $N A$ (Kuypers et al., 2004). A second explanation is that the axial resolution (see below) is distributed spatially inhomogeneously.

\section{Axial Resolution}

The axial resolution was investigated using fluorescent beads and thin homogeneous fluorescent layers.

The axial resolution of beads differed markedly in the three microscopes analysed. Generally, the FWHM values of the axial GAUssian fits (Fig. 34, lower row) clearly show (i) that the ApoTome's axial resolution is better than that of the epifluorescence microscope, (ii) that the ApoTome's axial resolution depends on the grid frequency $\nu$, and (iii) that the ApoTome's axial resolution with the VL-grid is about the same as that of the cLSM at a pinhole diameter of $d_{\mathrm{p}}=1$ A.U. In conventional epifluorescence, the bead images show the characteristic butterflyshaped sidelobes as predicted by the electromagnetic wave theory (Linfoot and Wolf, 1956). The sidelobes are clearly reduced in the ApoTome, enlarging the OTF and filling the "missing cone". If the finer grid (VL) is used, the ApoTome yields a $33 \%$ advantage over conventional epifluorescence and matches the cLSM's resolution.

Fig. 35 shows the axial resolution (i) of the cLSM as a function of the pinhole diameter, (ii) of the ApoTome equipped with the VH-grid $\left(\nu_{\tilde{\mathrm{V}} \mathrm{H}}=0.15\right)$, (iii) of the ApoTome equipped with the VL-grid $\left(\nu_{\tilde{\mathrm{V}} \mathrm{L}}=0.29\right)$, and (iv) of epifluorescence illumination as determined with beads (Fig. 35a) and layers (Fig. 35b). Referring 
a

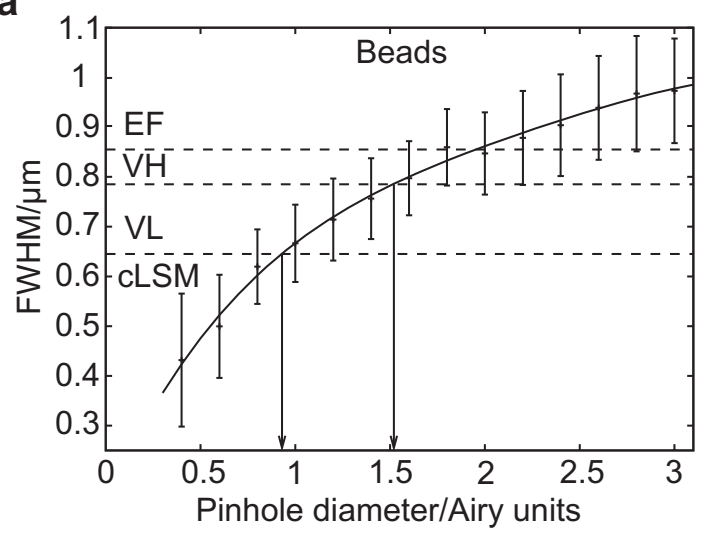

b

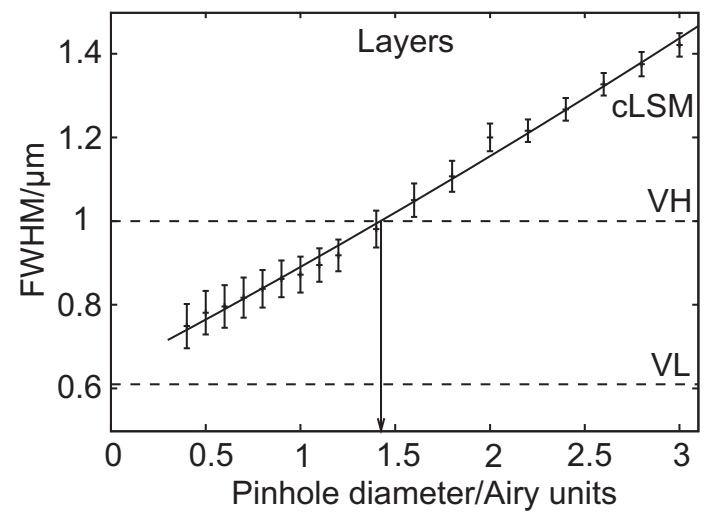

Figure 35: Axial resolution as a function of the pinhole diameter determined by the use of fluorescent beads (a) and subresolution homogeneous fluorescent layers (b). If the pinhole is opened, the axial resolution increases in a subproportional way in the case of using fluorescent beads. In contrast to that the axial resolution increases almost linearly if fluorescent layers are used. The diameter of the pinhole is given in A.U. as it appears back-projected in the focal region. For comparison, the ApoTome resolution values are given as indicated. $\mathrm{VH}$, ApoTome low sectioning $\left(\tilde{\nu}_{\mathrm{VH}}=0.15\right)$; VL, ApoTome high sectioning $\left(\tilde{\nu}_{\mathrm{VH}}=0.29\right)$; EF, conventional epifluorescence; cLSM, confocal laser scanning microscope. Objective, 63x/1.4 Plan-Apochromat.

to the measurements with beads, the coordinates of the beads were determined, and the same beads were used for all pinhole diameters. A likewise procedure was used for widefield imaging.

Using the ApoTome's VH-grid corresponds to $d_{\mathrm{p}}=1.5 \mathrm{~A}$.U. in the cLSM, while the VL-grid corresponds to $d_{\mathrm{p}}=1 \mathrm{~A}$.U. With increasingly smaller pinhole sizes, there is a notable increase in resolution in the cLSM though, e.g. $0.43 \pm 0.14 \mu \mathrm{m}$ at $d_{\mathrm{p}}=0.4$ A.U. This outperforms the ApoTome's best bead resolution. It can also be seen from Fig. $35 \mathbf{a}$ that for large pinhole sizes $\left(d_{\mathrm{p}} \geq 2\right.$ A.U.) the cLSM shows worse axial resolution than the epifluorescence microscope due to the GAUssian illumination of the BFP in the cLSM. The effect is that the effective $N A$ in the cLSM is lower than the nominal one.

If the axial resolution is determined with subresolution homogeneous fluorescent layers, the VL-grid of the ApoTome will yield about twice the resolution than could be achieved with the VH-grid. This could be expected from the doubled grid frequency and the corresponding cutoff-frequency. Under these conditions the ApoTome achieves a better resolution than the cLSM with a pinhole at minimum diameter (Fig. 35b). For these comparisons the mean resolution in the field-of-view was calculated. The bigger field-of-view of the cLSM was taken into account by cropping a field-of-view corresponding to the size of the ApoTome's field-of-view 

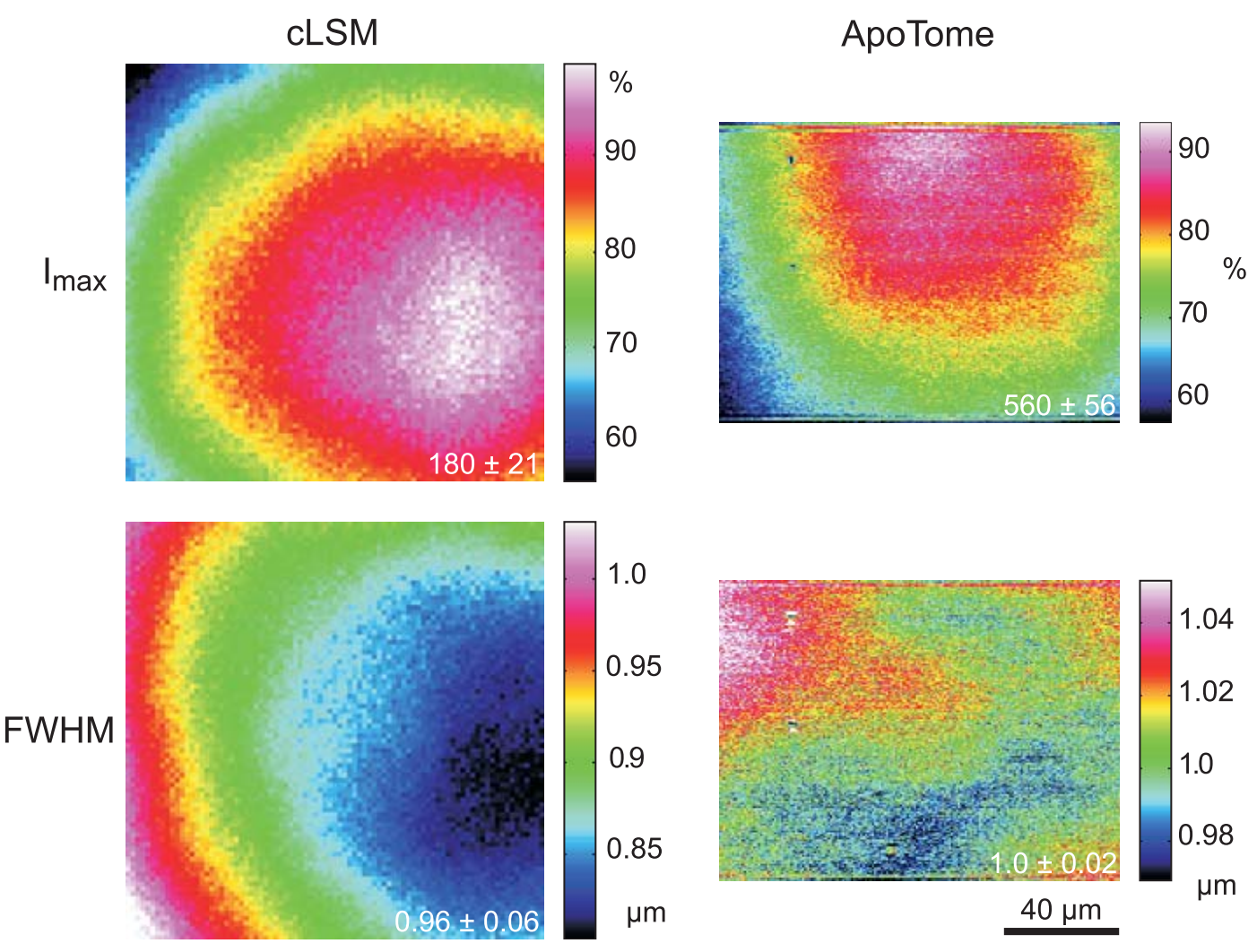

Figure 36: The extract of eSIPcharts shown above directly compares illumination and resolution properties of the ApoTome (VH-grid) and the cLSM system ( $d_{\mathrm{p}}=1 \mathrm{~A} . \mathrm{U}$.) The maximum intensity $I_{\max }$ has a circular-symmetric shape in both imaging modes, which is ascribed to chromatic and spherical aberration. Without the application of a spatial filter, there remain stripe artefacts induced by improper phase shifts of the grid, intensity fluctuations, stage movements during acquisition, and bleaching. The blots in the ApoTome-images stem from a residue on the grid, underlining the importance of keeping it perfectly clean. Values (insets) refer to binned data sets mean \pm sd over the whole field-of-view (intensity panel: ADU; resolution panel: $\mu \mathrm{m}$; ApoTome: $8 \times 2$ binning mask to conserve the stripe pattern of the $1388 \times 1040$ pixel image: one period of the grid pattern is sampled by $\approx 23$ pixel (VH-grid) and $\approx 11.5$ pixel (VL-grid), respectively, leading to an artefact stripe pattern of $3 \times$ the grid pattern; cLSM: $6 \times 6$ binning mask for a $512 \times 512$ pixel image); objective: $63 \mathrm{x} / 1.4$ Plan-Apochromat.

from the cLSM image. Since the axial resolution in the cLSM varied with the distance from the optical axis (see below), the centre line of the crop-stack was determined by the highest resolution.

In order to investigate the spatial distribution of the axial resolution, an eSIPchart was prepared as described in section 2. The eSIPchart intensity distribution clearly showed radial symmetry both for the cLSM (Fig. 36, upper left) and 
for the ApoTome (upper right). Moreover, the axial resolution in the cLSM was highly correlated with the radial intensity distribution (lower left). The symmetric shape can be ascribed to spherical and chromatic aberrations, while the off-axis resolution maximum can presumably be attributed to a slightly imperfect pinhole adjustment. It is remarkable that there is a difference of $\approx 250 \mathrm{~nm}\left(22 \%\right.$ or $\left.\frac{\lambda}{2}\right)$ between the best resolved regions at the intensity maximum and the worst resolved regions in the lower left corner. It follows from this that the radial symmetry of and the change in axial resolution indicates that the specific position in the field-of-view in a cLSM-image determines to which precision a subcellular structure can be resolved. Contrary to the cLSM, the ApoTome's axial resolution appears to be quite homogeneous, the deviations being $4 \%$ or $\leq \frac{\lambda}{10}$.

Interestingly, another feature of the ApoTome can be derived from the right panels in Fig. 36. Both subfigures reveal an artefact stripe pattern, which may result from i) stage vibrations leading to non-stationary imaging of the grid into the sample, ii) imprecise phase shifts of the grid, iii) bleaching of the grid pattern into the sample, iv) intensity fluctuations of the illumination arc source. The AxioVision software includes a digital filter that removes certain spatial frequencies in the Fourier domain. It was found that the so-called "weak-filter" removed the stripe artefacts best under most imaging conditions. This advantage is bought at a serious cost: the eSIPcharts produced with the "weak-filter"-setting led to a substantial loss in axial resolution, namely $1.20 \pm 0.03 \mu \mathrm{m}$ vs. $1.00 \pm 0.02 \mu \mathrm{m}(\mathrm{VH})$ and $0.69 \pm 0.05 \mu \mathrm{m}$ vs. $0.61 \pm 0.05 \mu \mathrm{m}$ (VL). Certainly it can be expected that spatial low-pass filtering reduces spatial resolution. As the AxioVision filtering algorithms are not documented, the user applying a spatial filter with the aim of removing stripe artefacts has to control the impact of the filter used on axial resolution empirically.

\subsubsection{Deep Tissue Imaging}

The resolution experiments discussed above solely demonstrate the resolution performance of the ApoTome under perfect conditions: the sample is attached to the cover slip, there is no fluorescence source outside the focal area, and the sample is stable enough to bear extended acquisition times. This is not the situation typically encountered in practical fluorescence microscopy of extended biological specimens. Therefore, I selected an in vivo tissue preparation to investigate the ApoTome's imaging and sectioning performance far $(>70 \mu \mathrm{m})$ from the cover slip within biological tissue, and compared it to the imaging performance of the cLSM 510.

Tissue preparation was performed as follows: Larvae of Xenopus laevis (stage 
54 and older, Nieuwkoop and Faber, 1994) were chilled in a mixture of ice and water and decapitated as approved by the Göttingen University Committee for Ethics in Animal Experimentation. A block of tissue containing the anterior two thirds of the olfactory bulb was extirpated and glued onto the stage of a vibroslicer (VT 1000S; LEICA, Bensheim, Germany). A tissue slice of $\approx 300 \mu \mathrm{m}$ thickness containing the glomerular layer was cut out and transferred to a patch clamp setup. The whole cell configuration was established on a mitral cell and it was loaded $\approx 1$ min with Alexa Fluor 488 biocytin (Probes). After another $20 \mathrm{~min}$, the tissue block was turned upside down and imaged under the ApoTome and the cLSM using an axial stepwidth of $1.5 \mu \mathrm{m}$ (overview) or $0.45 \mu \mathrm{m}$ (selected positions). Results are shown in Fig. 37.

Morphological details close to the surface of the tissue can be resolved equally well in the ApoTome and the cLSM (plane a of the figure), but in greater depths (plane $\mathbf{b}$ and $\mathbf{c}$ of the figure) the ApoTome images show increased blur and noise. In plane $\mathbf{b}$, for instance, the image plane cutting a dendrite is deeper than the cell's soma. The dendrite cut is clearly seen in both the ApoTome and the cLSM, but in addition, the ApoTome image shows a blurry spot which is not seen in the cLSM image, reporting the noise originating from the soma above that plane: this is because the ApoTome, though taking into account out-of-focus light while calculating an optical section, does not remove the noise of out-of-focus light. This is a common property in all widefield optically sectioning microscopes (Bewersdorf et al., 2006). At imaging depths of several cell layers, the noise component becomes dominant in the ApoTome, preventing the acquisition of feature-rich images, while at the same depth in the same preparation the cLSM can still extract tiny structures (plane c). This is of course due to out-of-focus photons being confocally suppressed in the cLSM. In contrast to that, at greater tissue depth in the ApoTome, the grid pattern projected onto the focus plane is increasingly superimposed by scattered light from above. Moreover, its in-focus modulation is correspondingly attenuated with stronger staining; these effects are not taken into account by Eqs. 22-24.

This experiment demonstrates that grid-SIM as implemented in the ApoTome is badly appropriate for imaging scattering tissue in greater tissue depths, since the noise contribution from fluorescence sources outside the focal area but inside the beam path will deteriorate the in-focus image and decrease the SNR. Strategies in SIM to enable deep tissue imaging include the illumination with patterns that have a much smaller mark-area ratio so that the amount of out-of-focus excitation will be decreased accordingly. Major disadvantages of such a strategy are the enormously prolonged recording time and the large amount of hard disk space required by a single data stack, not to mention the required processing power and 

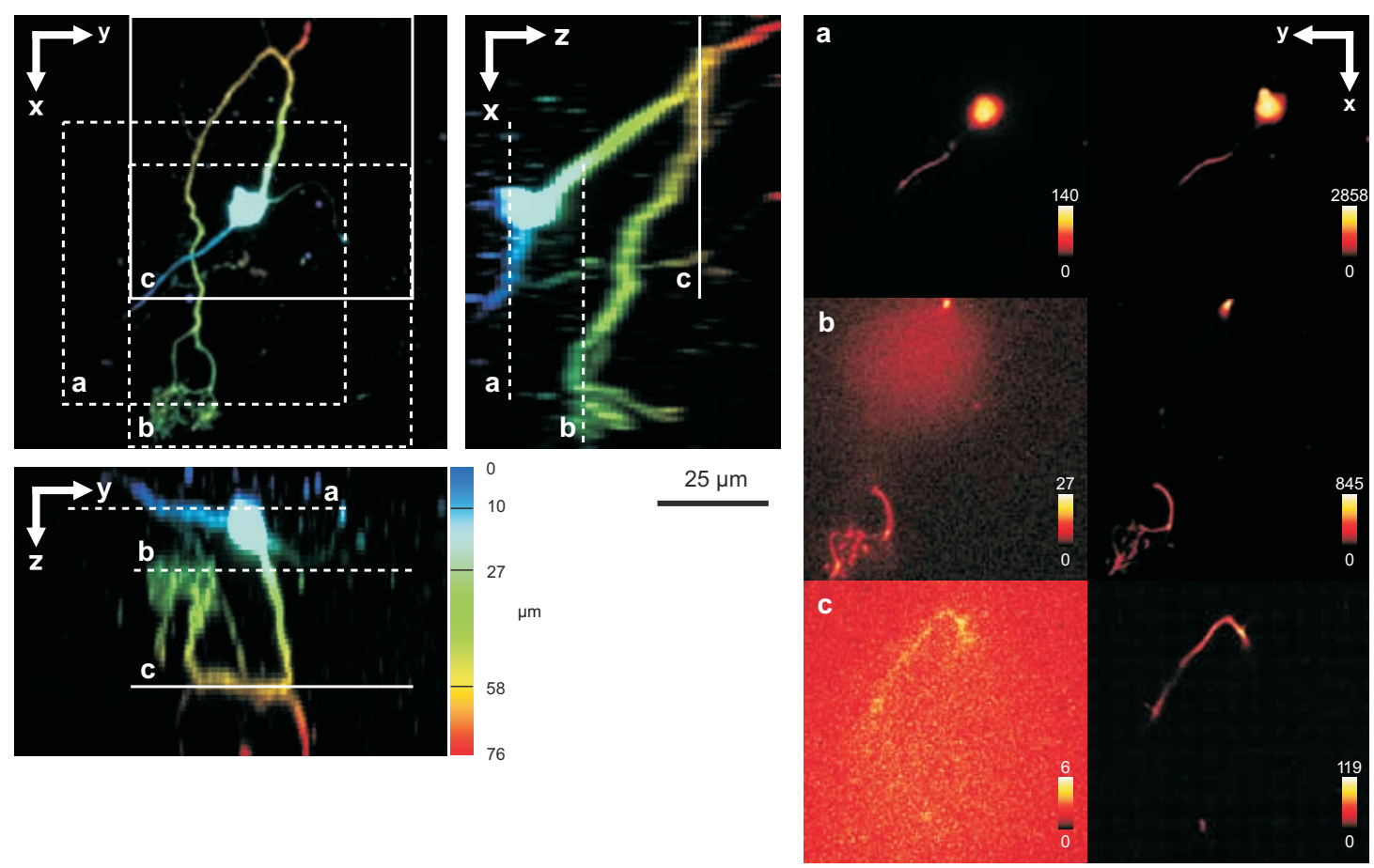

Figure 37: A mitral cell in the olfactory bulb of a Xenopus laevis tadpole loaded with Alexa-488 biocytin through a patch pipette. Left, maximum projections in $x y$ (top left), $x z$ (middle) and $y z$ (bottom left) calculated from a stack of cLSM-images. The colour is used to code the $z$-coordinate of maximal intensity (see colourbar). In addition, images were taken from the same tissue volume using the ApoTome. Right, comparison of ApoTome optical sections (left column of the six images to the right) and cLSM sections (right column) taken at the planes $\mathbf{a}, \mathbf{b}$, and $\mathbf{c}$ as indicated in the maximum projections ( $\mathbf{a}=10 \mu \mathrm{m}, \mathbf{b}=27 \mu \mathrm{m}, \mathbf{c}=58 \mu \mathrm{m})$. At $z=27 \mu \mathrm{m}$, the ApoTome image is markedly corrupted from noise caused by the brightly stained soma above the focal plane. If the ApoTome is used for imaging at greater depths, the noise will become dominant with increasingly low contrast (see scalebar), while the cLSM still yields high contrast images with a contrast nearly as high as in the ApoTome at $z=10 \mu \mathrm{m}(\mathbf{a})$.

time (Heintzmann, 2006). The test sample is suited well for a comparison of the sectioning property of an optically sectioning microscope. Most studies in literature restrict themselves to a comparably small axial range (e.g. Bewersdorf et al., 2006), because there is no standard test specimen for testing sectioning performance deep inside a scattering tissue sample. Here it was shown how the sectioning performance of the ApoTome rapidly deteriorates with increasing tissue depth, and that it is massively corrupted by out-of-focus fluorescence noise contributions. Despite these strong arguments for the preparation, it also bears some disadvantages:

- as it is a living non-fixed sample, it tends to bleach upon prolonged exposure. It can only be used once and has to be freshly prepared for each experiment. 
- a microinjection setup with the respective know-how needs to be available.

- an appropriate tissue preparation needs to be available. Only few tissues, mainly within the nervous system, feature selectively markable small extended structures like dendrites.

For these reasons a stable fixed sample is required for a generalised approach on deep tissue optical sectioning performance testing.

\subsubsection{Anisotropy}

The SIM-technique employed in the ApoTome is highly anisotropic because the grid pattern is one-dimensional and shifted rather than rotated. It has been shown that anisotropic SIM can lead to anisotropic lateral resolution (Karadaglić, 2004; Karadaglić and Wilson, 2008). It has not been shown yet whether this effect plays a significant role in biological applications. Furthermore, the anisotropy in axial resolution has not been treated. In general, there are lots of linear structures in biological tissues that might be resolved anisotropically, e.g. in the cytoskeleton, endoplasmatic structures, muscle fibres, or nerves. All of these can be selectively fluorescence labelled. If their size is below the resolution limit, they present a valuable probe for testing the anisotropy effect in biological specimens, especially since one has the free choice for the employed fluorescence marker, as long as it is sufficiently stable, selective, and dense enough on the structure under observation.

Mitral cells of Xenopus laevis tadpoles extend straight dendrites to neighbouring cells in culture, which is why the decision for a calcein-labelled probe of dendrites in culture was made. Dendrites bear several pitfalls in experiments which have to be circumvented:

- not all dendrites are smooth and straight. A visual selection of appropriate dendrites is required.

- dendrites may disintegrate during the experiment, so it has to be repeated with another dendrite.

- if the illumination takes too long, the fluorophore will bleach.

- the dendrite might not be attached to the coverslip, thus inducing unwanted bias by the refractive index mismatch between the immersion water and the Ringer solution. By cautiously knocking at the microscope, loosely hanging dendrites can be distinguished from attached ones.

- the dendrite might be too big, meaning that it is bigger than the resolution limit. As the lateral resolution is not changed in this type of SIM, it served 
as a measure for the resolution limit; in the evaluation only those dendrites were used whose lateral resolution was at the resolution limit.

Cell culture preparation was done as described by Gennerich and Schild, 2002. Measurements were carried out within 5 to 10 days after plating. Prior to the experiments, the cultures were loaded for $15 \mathrm{~min}$ with $100 \mu \mathrm{M}$ calcein-AM (PROBES, $\lambda_{\mathrm{em}}=514 \mathrm{~nm}$ at $\left.\mathrm{pH}=7.8\right)$, which was dissolved in dimethylsulfoxide (DMSO) and Pluronic F-127 (Probes). The final concentrations of DMSO and Pluronic F-127 did not exceed $0.5 \%$ and $0.1 \%$, respectively. Dendrites were viewed through the microscope's eyepiece, then they were selected and turned parallel (p) or orthogonal (o) to the grid by turning the culture dish. A perfect $\mathbf{p}$ or o-positioning could not be achieved, which turned out to be beneficial in the evaluation since the dendrite's measured extension is insensitive to slight deviations with respect to the o-positioning, but highly sensitive to the p-positioning. Hence the angle $\alpha$ between dendrite and grid pattern was taken as parameter (Fig. 38). A 2D-GAUssian fit was applied to the image plane corresponding to the orientation of the dendrite (either $x z$ or $y z$-plane). The systematic error that appears in the lateral dimension if slightly tilted dendrites are fitted (i.e. the adjacent leg rather than the hypotenuse is fitted) is marginal and can be neglected: for $\alpha=3^{\circ}$, the error is $<2 \%$. The lateral extensions of the dendrites are virtually independent of their orientation with respect to the grid $(350 \pm 5 \mathrm{~nm}(\mathbf{p})$ and $347 \pm 5 \mathrm{~nm}(\mathbf{o})$, mean $\pm \mathrm{sd})$. The axial extension of $\mathbf{p}$-dendrites in contrast is significantly larger than that of $\mathbf{o}$-dendrites. The angle dependency of the axial resolution is rather steep: at $\alpha=2.8^{\circ}$, the apparent axial extension of the $\mathbf{p}$-dendrite is nearly equal to the axial extension of the o-dendrite (Fig. 38e).

Karadaglić, 2004 calculated the object transfer function for grid projection SIM. The effective object transfer functions are shown in Figs. 39 and 40. In these figures, $\nu \widehat{=} \tilde{\nu}$ for convenience. It can be seen that the anisotropy introduced by the grid illumination exhibits severe differences in the imaging of lateral structures of similar dimension like the grid frequency. Being more explicit, structures oriented parallel to the illumination pattern are selectively enhanced if slightly out-of-focus, i.e. they appear brighter. Lateral resolution anisotropy did not play a role in the preparation used though, because the dendrites' lateral spatial frequency is $>\tilde{\nu}$.

It has been shown that grid-SIM exhibits anisotropic resolution, both laterally and axially. In the latter case it does not play an important role in biological applications unless some structures happen to be oriented almost perfectly parallel to the illumination grid. In the first case, structures must have spatial frequencies $<\tilde{\nu}$. That is the reason why it cannot be observed if point objects or sparsely 

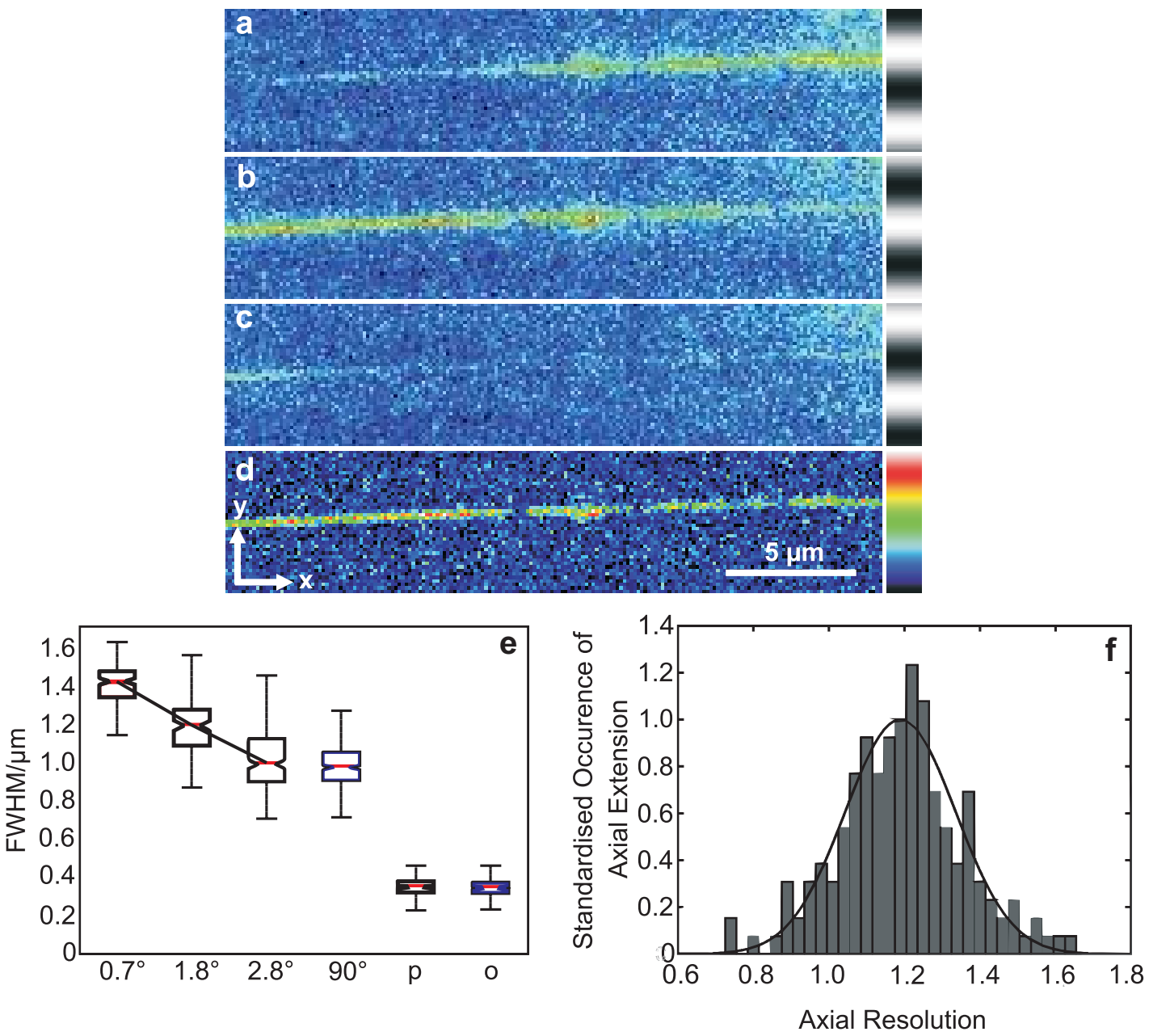

Figure 38: Anisotropic resolution in the ApoTome. Straight-grown smooth fluorescencelabelled dendrites from cell cultures of olfactory bulb neurons of Xenopus laevis tadpoles were recorded as image stacks at several angles to the grid pattern. In the example shown (a-d), the angle is $2.8^{\circ}$. a-c), raw images, right: illumination pattern giving the image to the left, d), processed image, right: colourbar for a-d). e), an analysis of each dendrite reveals a rapidly increasing resolution with increasing angle to the grid. The lateral extensions of the dendrites in the $x y$-plane does not depend on the angle between the dendrite and the grid. It was $350 \pm 5 \mathrm{~nm}$ (mean $\pm \mathrm{sd}$ ) in case the dendrite was parallel to the grid and $347 \pm 5 \mathrm{~nm}$ if it was orthogonal to the grid. f), normalised distribution of the axial extension of the dendrite tilted $1.8^{\circ}$ obtained from fits to the $x z$-plane. The GaUssian distribution of the resolution observable supports the hypothesis that the dendrites' extensions are below the resolution limit.

stained structures are imaged, but it will become important if intensively stained tissue slices or cell somata in cultures are imaged. The only way to avoid anisotropic imaging is to rotate the grid in addition to shifting it. The required recording time will then multiply accordingly, because more images are required to obtain 

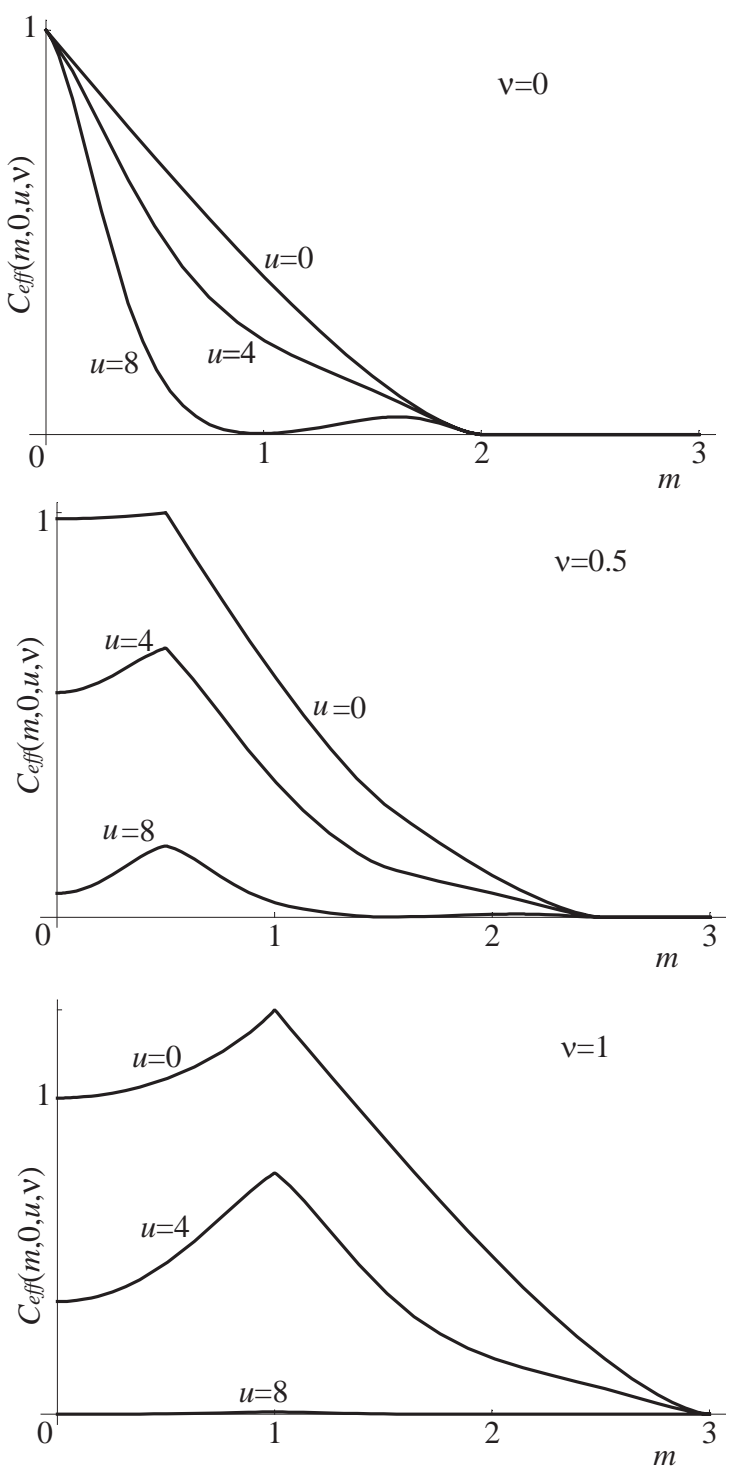

Figure 39: Effective optical transfer function $C_{\text {eff }}(m, n=0, u, \nu)$ in grid-SIM for various values of $\nu: \nu=0 \hat{=}$ conventional microscopy, $\nu=0.5 \hat{=}$ best possible case in the ApoTome, $\nu=1$ 列 general optimal case. $m$, spatial frequency parallel to the grid-direction. In case of defocus (i.e. $u>0)$, object features around the frequency $(m, n)=(\nu, 0)$ show significant enhancement.( Figure taken from Karadaglić, 2004.)

one optically sectioned image. This abolishes one advantage of grid-SIM over full field confocal imaging. Grid-SIM also requires the object to be stable (spatially and temporally) for the complete recording time, making the acquisition of an isotropically resolved object a demanding task.

The test object I used is suited well for the purpose of unravelling axial resolution anisotropy because it was below the resolution limit. It was a very good choice with regard to its practical relevance, as it reflects well the situation a microscopist 

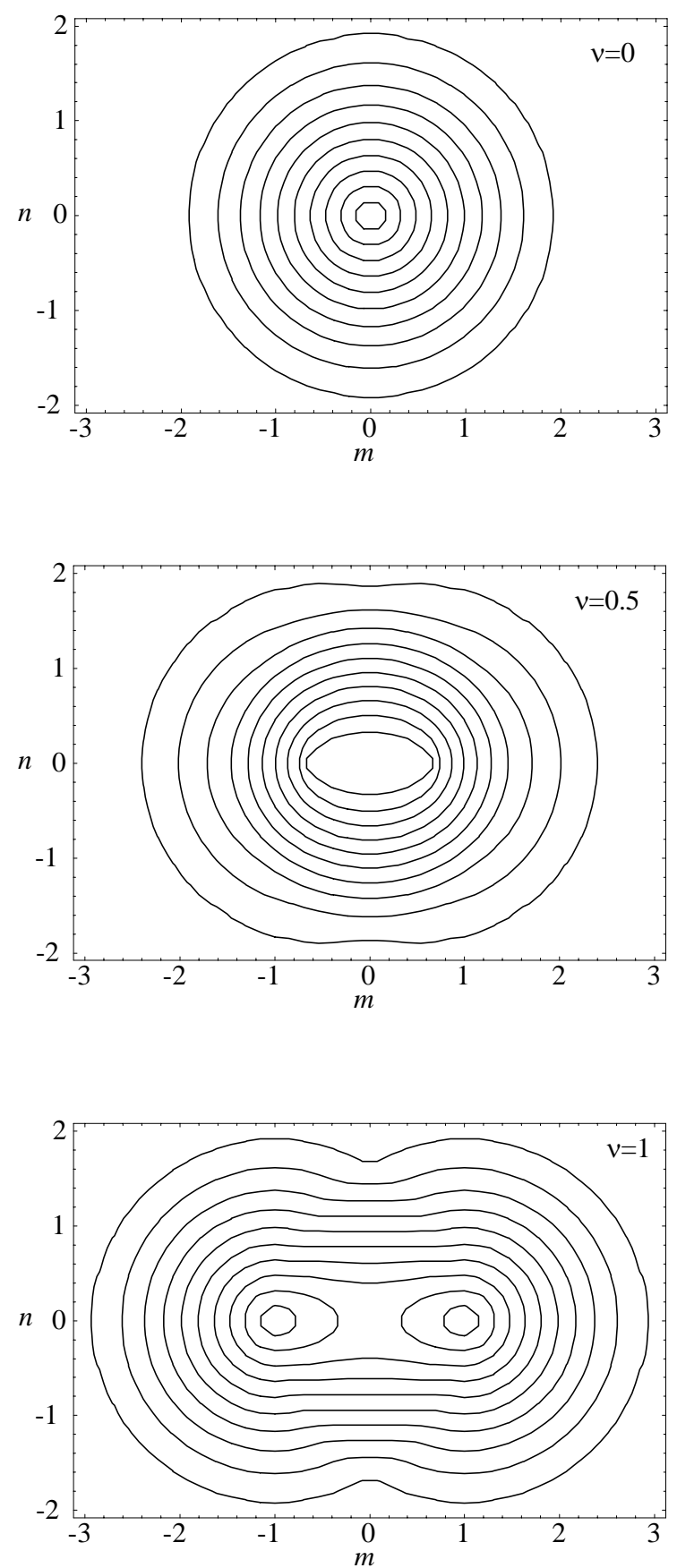

Figure 40: Effective optical transfer function $C_{\text {eff }}(m, n, 0, \nu)$ in grid-SIM for $\nu=0,0.5$, and 1. $m, n$, spatial frequencies parallel and orthogonal to the grid direction. Sectioning becomes severely anisotropic if samples are imaged that feature structures $\leq \nu$ that are oriented parallel to the grid pattern. (Figure taken from Karadaglić, 2004.)

in biology encounters frequently in lab routine. It has to be pointed out that by imaging this object, it is not possible to detect additional anisotropy effects that relate to the grid frequency $\tilde{\nu}$. For this purpose, a fluorescence test sample featuring lines with line weights around $\tilde{\nu}$ for each objective/grid combination 
would be beneficial.

\subsubsection{Spectral Versatility}

An advantage of widefield SIM compared with confocal microscopy is that it can make use of the whole spectral range from $300 \mathrm{~nm}$ (limited by the UV-transmission of the optical components and the emission spectrum of the arc lamp) to $\approx 1000 \mathrm{~nm}$ (limited by the IR-transmission of the optical components, the sensitivity of the detector, the emission efficiency of the light source, and the onset of IR-absorption of water) by using an arc lamp as illumination source. Hence it can make use of the whole spectral range. In contrast to that, the cLSM is limited to the available laser transitions and lasers. Here it is shown that widefield SIM cheaply produces optical sections over a spectral range that is hardly - if not impossible - and very expensive to access with lasers. The quality of a grid-SIM optical section is contrasted to conventional epifluorescence.

Multicolour tissue visualisation was prepared in four subsequent steps. (i) For tracing olfactory nerve fibres, crystals of Alexa-488 biocytin were placed into the cut olfactory nerve of chilled tadpoles, and the lesion was closed with cyanoacrylic glue (Roti-Coll 1, Roth, Karlsruhe, Germany). The tadpole was decapitated three hours later as described above, and a block of tissue containing the olfactory nerves and the anterior two thirds of the brain were cut out and kept in bath solution. (ii) A solution containing Alexa-546-biocytin was injected into the olfactory tract, and the tissue was incubated for another two hours. Then the tissue block was fixed in $4 \%$ formaldehyde in $0.1 \mathrm{M}$ PBS for $\approx 2 \mathrm{~h}$, washed in PBS $(3 \times 10 \mathrm{~min})$, embedded in agarose, glued onto the stage of a vibroslicer (VT 1000S; LEICA, Bensheim, Germany) and cut into slices of $70 \mu \mathrm{m}$ thickness. (iii) The slices were incubated in PBS containing 1\% BSA for $30 \mathrm{~min}$ at room temperature. Then they were incubated overnight in Alexa Fluor 647 Phalloidin (PROBES) ( $5 \mu \mathrm{l}$ from methanol stock solution) in $200 \mu \mathrm{l}$ PBS with $1 \%$ BSA. (iv) For further staining with DAPI (Probes), the slices were washed $3 \times 10 \mathrm{~min}$ in PBS and incubated for $15 \mathrm{~min}$ in a mixture of $1 \mathrm{ml}$ PBS and $2 \mu \mathrm{l}$ of DAPI stock solution. After washing them in PBS $5 \times 10$ min, they were mounted on object slides with DAKO CYTOMATION (Hamburg, Germany) Fluorescence Mounting Medium S3023.

\section{Solutions}

Bath solution The composition of the bath solution was (in $\mathrm{mM}$ ): $\mathrm{NaCl}, 98$; $\mathrm{KCl}, 2 ; \mathrm{CaCl}_{2}, 1 ; \mathrm{MgCl}_{2}, 2$; glucose, 5; sodium pyruvate, 5; HEPES, 10. The $\mathrm{pH}$ was adjusted to 7.8 , since this is the physiological $\mathrm{pH}$ in this poikilothermal species (Howell et al., 1970). 

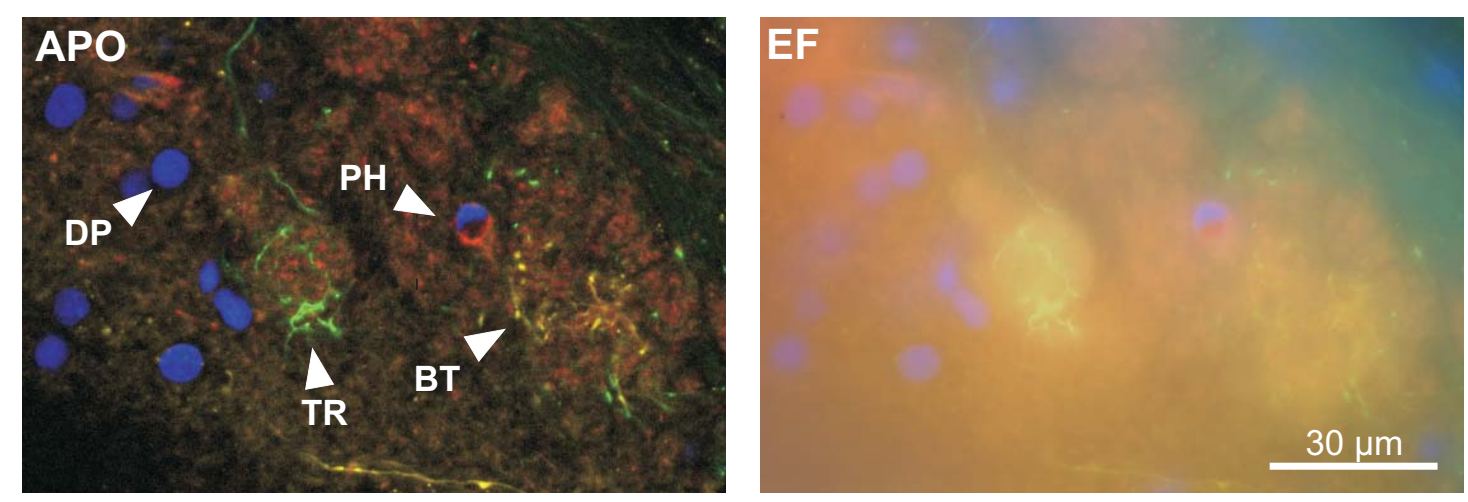

Figure 41: A $70 \mu \mathrm{m}$ thick tissue block of the olfactory bulb of a Xenopus laevis tadpole: blue: nuclei (DAPI), green: axons (Alexa Fluor 488 tracing), yellow: dendrites (Alexa Fluor 546 biocytin backtracing), red: actin-filaments (Alexa Fluor 647 phalloidin immunostaining). Left, ApoTome image. Right, conventional epifluorescence image. The sectioning makes it possible to clearly separate the four colour channels. The axonal innervation of two glomeruli and the dendritic innervation of one of them can be seen. To a certain extent, the phalloidin-staining is present in the whole tissue and especially in the two blood vessels included in the image, whereas the DAPI-staining reveals that there are few cells in the glomerular layer. Erythrocytes show autofluorescence in the DAPI-channel.

Pipette solution The composition of the intracellular solution was (in $\mathrm{mM}$ ): $\mathrm{NaCl}, 2 ; \mathrm{KCl}, 11 ; \mathrm{MgSO}_{4}$, 2; K-gluconate, 80; HEPES, 10; EGTA, 0.2; $\mathrm{Na}_{2} \mathrm{ATP}, 2$; $\mathrm{Na}_{2} \mathrm{GTP}, 0.2$. The osmolarity was adjusted to $190 \mathrm{mOsm}$, and the $\mathrm{pH}$ to 7.8 .

PBS The composition of the 0.1 M PBS-solution was (in $\mathrm{mM}$ ): $\mathrm{NaCl}, 27.4 ; \mathrm{KCl}$, 0.54; $\mathrm{Na}_{2} \mathrm{HPO}_{4}, 1.6 ; \mathrm{KH}_{2} \mathrm{PO}_{4}, 0.28$. The $\mathrm{pH}$ was adjusted to 7.4.

All chemicals required for the solutions above were purchased at SIGMA (Deisenhofen, Germany).

Fig. 41 clearly demonstrates that optical sectioning is required even if thin tissue sections are investigated: otherwise it is not possible to remove background fluorescence and thus assign the fluorescence contributions to their respective emitters properly.

\subsection{Discussion}

It has been shown that structured illumination microscopy, as it is implemented in the ApoTome, is capable of optical sectioning and hence superior to conventional epifluorescence microscopy in most fluorescence applications. Conventional epifluorescence would be favoured (i) if speed matters, because it is immanently at least 
three times faster than grid-SIM, (ii) if the sample is not stable over the acquisition time, (iii) at very low signal levels, because many more photons are required in SIM to achieve a similar SNR like in conventional epifluorescence, (iv) if the sample is sparsely labelled and optical sectioning is not required. The thickness of the optical section or the amount of out-of-focus light removed depends directly on the pitch of the illumination grid used. Even if the gain in resolution is marginal compared with widefield epifluorescence, grid-SIM can still remove out-of-focus contributions.

Although resolution improvement is not as important as optical sectioning in lab routine, it can serve as a valuable comparator of microscope performance as I already pointed out in section 2. Taking the resolution as observable, certain peculiarities of the systems under investigation were revealed that would have remained unnoted otherwise. First, the lateral resolution showed that the laser illumination with its GAUSsian illumination profile causes the cLSM to yield a lateral resolution worse than achieved with widefield epifluorescence. Second, the axial resolution determined under standard conditions (high NA, ApoTome grid VH, cLSM pinhole diameter $d_{\mathrm{p}}=1 \mathrm{~A}$.U.) prefers the cLSM. If non-standard conditions are used (ApoTome grid VL, cLSM closed pinhole), the ApoTome can exceed the cLSM in sectioning power in certain niche applications, namely if it is required to image contiguously stained thin samples. Third, the resolution distribution unveiled a rather isotropic axial resolution in the ApoTome, whereas it was central-symmetric in the cLSM. As a consequence, the determination of the point of optimal resolution would be mandatory for experiments requiring high resolution, since it does not necessarily coincide with the optical axis. Fourth, the imaging in the ApoTome was shown to be anisotropic for spatial frequencies in the sample around $\tilde{\nu}$, considering brightness and resolution. From this it follows that in some ambiguous cases where the sample contains such frequencies, a change of the illumination grid, the magnification, or the orientation of the sample might help.

Imaging deep inside scattering tissue is not the domain of grid-SIM. Noise contributions from out-of-focus fluorescence inhibit imaging with an acceptable SNR. For imaging deep inside scattering tissue, techniques are preferred that either physically block out-of-focus light (cLSM) or that do not allow out-of-focus light to be generated in the first place (TPA).

In a nutshell, the ApoTome is an option to be considered if mainly thin samples that are multiply labelled (e.g. histological sections) need to be imaged. To avoid artefacts, these samples have to be stable on a timescale of at least $1 \mathrm{~s}$, since this is the minimal time required for the acquisition of three raw images at present. 
Imaging speed in the ApoTome can be improved (i) by the use of faster CCDcameras such as EM-CCDs, (ii) by using brighter illumination sources, e.g. laser diodes, (iii) by using off-line computation of the optical sections, (iv) by using a constantly moving grid, because it takes the grid some time to relax if it has to be fixed for acquisition (Chasles et al., 2007).

\subsection{Appendix: Artefacts in Grid-SIM}

SIM in general and grid-SIM as implemented in the ApoTome is prone to a variety of imaging artefacts which have been discussed by Cole et al., 2000; Schaefer et al., 2004 and Karadaglić, 2004. Most of these artefacts result in a residual stripe pattern of the grid frequency $\tilde{\nu}$ and higher harmonics. The AxioVision software offers a filter to remove higher harmonics, but as this is done at the cost of axial resolution it cannot be advised. Reasons for residual stripe patterns in the processed image include:

- fluctuations of the illumination source. A normalisation of the images prior to subsequent processing helps, but it is more important to guarantee a stable light source and let it warm up before the imaging.

- improper grid positioning. If the grid position can be exactly determined from the raw images, the final image can also be obtained by calculating

$$
I_{\mathrm{s}}=\left|I_{1}+I_{2} \mathrm{e}^{-\mathrm{i} \frac{2 \pi}{3}}+I_{3} \mathrm{e}^{\mathrm{i} \frac{2 \pi}{3}}\right|
$$

rather than using Eq. 23.

- a non-sinusoidal illumination pattern results in higher harmonics being present in the processed image. It can be avoided by using a high $\tilde{\nu}$, so that higher harmonics are beyond the cutoff-frequency, and by Fourier-filtering of the image.

- sample movement due to mechanical and thermal drift. This should be avoided by all means.

- sample bleaching. If only one dye is used, a normalisation might help. If several dyes with different bleaching constants are used, artefact removal becomes difficult or even impossible with present techniques, since the contribution of each fluorophore to each pixel would need to be determined by spectral unmixing.

- pattern bleaching. In observation mode, the grid is sinusoidally moved to avoid pattern bleaching. Once the acquisition is started, half of the specimen 
is illuminated for $\frac{2}{3}$ of the three image acquisition time, whereas the other half is illuminated for $\frac{1}{3}$ of the total acquisition time. This inevitably creates patterned bleaching with $3 \tilde{\nu}$ and corresponding artefacts in the processed images (see Fig. 31). In spatial and temporal image stack acquisitions, this could be avoided to a certain extent by shifting the start phase of the grid with each new image.

In addition, the ApoTome grid has to be changed manually unlike the fully automated pinhole in the cLSM. This results in exposing the grid's sensitive optical surface to dirt and damage. As the grid is in a conjugated image plane, dirt on it heavily corrupts the actual image. Therefore, a mechanical implementation of grid-SIM comparable to a filter wheel with several encapsulated grids with various spacings mounted would be a promising improvement. An even more sophisticated method was presented by Monneret et al., 2006: they use a programmable liquidcrystal (LC) light modulator which is inserted into the illumination path to select the spatial frequency of the illumination pattern freely. The major disadvantages compared with solid-grid-implementations are that illumination intensity is wasted, and that the LC-pixels are of limited size. 


\section{Summary}

The development of microscopes that can record fluorescence intensity in five and more dimensions in one experiment (e.g. space, time and colour) makes parameters available for the scientific community that were previously beyond reach. A new realm of possibilities is opened by the observation of correlations between these dimensions. The goal of front-end microscopy is the extraction of quantitative information from certain correlations. In fluorescence recovery after photobleaching, for instance, diffusion coefficients are calculated from the correlation of space and time. In FöRSTER resonance energy transfer imaging molecular interactions and concentrations are determined, and space and colour correlated.

To render the correlations that are determined in these experiments as valid, the recording system needs to be thoroughly calibrated. Otherwise artefacts arise that can lead to wrong conclusions, and the quantitation of the parameters is impossible. The first part of this thesis was determined to alleviate quantitation and optimisation in optically sectioning microscopy. For this purpose the new tool for the assessment of imaging quality in optically sectioning microscopes, the sectioned image property chart (SIPchart), was fundamentally re-engineered obtaining the extended SIPchart (eSIPchart). SIPcharts are created by the analysis of an axial image stack of a thin uniform fluorescent layer. A pixelwise fit to the axial intensity distributions yields parameter maps that describe a certain imaging property in the field-of-view, e.g. field-dependent intensity variations.

The eSIPchart routine was developed in the course of this thesis to analyse axial image stacks of fluorescent solutions. It implements the following: a statistical analysis is performed with which arbitrary detector units are converted to real photon numbers. These allow the evaluation of the goodness-of-fit with a $\chi_{\mathrm{R}^{-}}^{2}$ parameter map. They also enable the creation of concentration calibration curves. The implemented VoIGT-fit gives a weight factor, on whose basis the quality of the illumination can be estimated.

Great benefit can be taken from the transition from the recording of thin layers to fluorescent solution: i) the same dye can be used for calibration and for imaging. Hence calibration errors caused by deviating wavelengths and refractive indexes of the test sample, i.e. a thin layer, can be excluded. ii) fluorescent dyes can be found in places where fluorescence microscopy is performed. They are easily available, cheap, and versatile. iii) a fluorescent solution can contain several dyes and thus 
cover a big spectral range. iv) the refractive index of the solvent can be adjusted so that it matches the refractive index of the immersion medium. With the data obtained from eSIPchart analysis, a thorough calibration can be performed for chromatic and spherical aberrations and those which origin from the setting of the correction collar. Finally the eSIPchart gathers the characteristics of a particular setting in a microscope in a comprehensive manner. It thus becomes possible to judge upon the imaging properties and the suitability of an optically sectioning fluorescence microscope for a designated experiment at a glance.

The second part of the thesis deals with the application of a set of custom evaluation tools for the comparison of three microscopy systems. The ApoTome, a linear grid structured illumination microscope, is opposed to the confocal laser scanning microscope (cLSM), and conventional widefield epifluorescence microscopy. Different parameters of imaging are assessed with different techniques: the axial and lateral resolution is measured with subresolution fluorescent beads and thin uniform fluorescent layers. The field dependency is evaluated with eSIPcharts. The resolution anisotropy is measured by using linear dendrites from cell cultures which were prepared from olfactory bulb neurons of the larva of Xenopus laevis. Deep tissue imaging is evaluated eventually by using microinjected mitral cells in the olfactory bulb of the same animal.

It was found out that the ApoTome achieves a better lateral resolution than a cLSM in standard imaging conditions, that is with a confocal pinhole diameter of $d_{\mathrm{p}}=1 \mathrm{~A} . \mathrm{U}$. in the cLSM and the recommended grid "VH" in the ApoTome. This is because the ApoTome's backfocal plane (BFP) is illuminated homogeneously, whereas the cLSM's BFP is illuminated with the clipped GAUssian intensity distribution of a laser. The axial resolution is better in the cLSM than in the ApoTome with one exception: the ApoTome exceeds the cLSM in the resolution of low spatial frequencies recorded at optimal resolution conditions (cLSM, $d_{\mathrm{p}}=$ 0.4 A.U. and ApoTome, "VL"-grid).

The axial resolution in the cLSM has a central-symmetric distribution over the field-of-view, mainly due to scanning-related aberrations, whereas the ApoTome's axial resolution appears to be homogeneously distributed over the field-of-view.

Grid-structured illumination microscopy features anisotropic resolution and selective enhancement of structures in the sample that have the same orientation and frequency as the grid.

Structured illumination microscopy is restricted to thin specimens because PoISSONian noise contributions from out-of-focus fluorescence cannot be removed. In addition to that, the sample needs to remain stable for at least some seconds since the acquisition of the raw data for one optically sectioned image lasts $\approx 1 \mathrm{~s}$. 


\section{Zusammenfassung}

Die Entwicklung von Mikroskopen, die innerhalb eines Experiments Fluoreszenzintensitäten in mehr als fünf Dimensionen aufnehmen können (z. B. Raum, Zeit und Farbe), ermöglicht das Messen von Parametern, die zuvor nicht zugänglich waren. Viele weitere experimentelle Fragestellungen ergeben sich durch die Untersuchung der Korrelationen zwischen diesen Dimensionen. Das Ziel moderner Mikroskopie ist die Extraktion quantitativer Informationen aus bestimmten Korrelationen. Beispielsweise nutzt man in der sog. Fluoreszenz-Wiederherstellung nach Photobleichung (fluorescence revovery after photobleaching, FRAP) die Korrelation zwischen Zeit und Raum zur Bestimmung von Diffusionskoeffizienten. Im FÖRSTER-Resonanzenergietransfer werden Raum und Farbe zur Bestimmung von molekularen Wechselwirkungen und Konzentrationen miteinander korreliert.

Die bildgebenden Systeme müssen sorgfältig kalibriert werden, damit die Korrelationen, die in derartigen Experimenten untersucht werden, die zugrunde liegenden Mechanismen wiederspiegeln. Andernfalls analysiert man Artefakte, die zu falschen Schlüssen (ver-)leiten, und eine Quantifizierung der Parameter erweist sich als unmöglich. Der erste Teil dieser Dissertation hat zum Ziel, die Quantifizierung und Optimierung in der optisch schnittbildenden Mikroskopie zu vereinfachen. $\mathrm{Zu}$ diesem Zweck wurde das neue Werkzeug zur Beurteilung der Bildqualität schnittbildender Mikroskope, das sog. sectioned image property chart (SIPchart), fundamental überarbeitet, so dass man ein erweitertes SIPchart (eSIPchart) erhält. SIPcharts erhält man durch die Analyse eines axialen Bildstapels einer extrem dünnen $(\leq 100 \mathrm{~nm})$, gleichförmigen fluoreszierenden Schicht. Durch die pixelweise Modellierung der axialen Intensitätsverteilungen erhält man Parameter-Karten, die eine bestimmte Abbildungseigenschaft im Bildfeld beschreiben, wie zum Beispiel ortsabhängige Intensitätsschwankungen.

Im Rahmen dieser Dissertation wurde die eSIPchart-Routine zur Analyse axialer Bildstapel von fluoreszierenden Lösungen entwickelt. In dieser werden folgende Erweiterungen eingeführt: Auf der Grundlage statistischer Analyse werden „zufällige Detektoreinheiten“ in die Anzahl detektierter Photonen umgerechnet. Dies ermöglicht die Beurteilung der Qualität der Modellfunktion über eine Karte des $\chi_{\mathrm{R}}^{2}$-Parameters. Weiterhin kann man nun Konzentrations-Kalibrations-Kurven aufnehmen. Die neu eingeführte VOIGT-Funktion liefert einen Wichtungsfaktor, aufgrund dessen die Ausleuchtung der Rückapertur abgeschätzt werden kann. 
Der Schritt von dünnen Schichten zu fluoreszierenden Lösungen ist aus verschiedenen Gründen von Vorteil: i) Für Kalibration und Experiment kann derselbe Farbstoff verwendet werden. Somit werden Kalibrationsfehler vermieden, die durch ein unterschiedliches Fluoreszenzspektrum oder einen Brechungsindexunterschied des Testpräparates, wie es eine dünne Schicht darstellt, zustande kommen. ii) Fluoreszenzfarbstoffe sind in jedem Labor vorhanden, in dem Fluoreszenzmikroskopie betrieben wird. Sie sind leicht zu erwerben, billig und vielseitig. iii) Eine fluoreszierende Lösung kann mehrere Farbstoffe enthalten, die somit ein großes Fluoreszenzspektrum abdecken. iv) Der Brechungsindex des Lösungsmittels kann auf den Brechungsindex des Immersionsmediums eingestellt werden. Die eSIPchart-Analyse ermöglicht eine präzise Charakterisierung der sphärischen und chromatischen Aberrationen sowie eine Optimierung der Einstellung des Korrekturringes qualitativ hochwertiger Mikroskopobjektive. Abschließend werden die Charakteristika einer bestimmten Mikroskopkonfiguration im eSIPchart übersichtlich zusammengestellt. Das ermöglicht die schnelle Beurteilung der Abbildungseigenschaften sowie der Eignung eines optisch schnittbildenden Mikroskops für ein geplantes Experiment.

Der zweite Teil der Dissertation behandelt die Anwendung einer Reihe neu entwickelter Vergleichsmethoden zum Vergleich von drei Mikroskopsystemen. Das ApoTome nutzt zur optischen Schnittbildung strukturierte Beleuchtung durch ein lineares Gitter. Dieses wird einem konfokalen Laser-Raster-Mikroskop (cLSM) und konventioneller Weitfeld-Epifluoreszenzmikroskopie gegenübergestellt. Verschiedene Abbildungsparameter werden durch verschiedene Methoden bestimmt: Die axiale sowie die laterale Auflösung werden mit fluoreszierenden Kügelchen und dünnen fluoreszierenden Schichten bestimmt. Die Ortsabhängigkeit verschiedener Parameter wird durch eSIPcharts ermittelt. Die Auflösungsanisotropie wird mittels linear gewachsener Dendriten ermittelt. Diese stammen aus Zellkulturen aus dem Riechhirn der Kaulquappe des afrikanischen Krallenfrosches Xenopus laevis. Das Abbildungsverhalten für tief im Gewebe liegende Strukturen wird vermittels mikroinjizierter Mitralzellen aus dem Riechhirn des Krallenfrosches untersucht.

Das ApoTome erreicht unter Standardbedingungen, d. h. mit einem Durchmesser der konfokalen Lochblende von $d_{\mathrm{p}}=1$ A.U. und dem ApoTome-Gitter „VH“, eine bessere laterale Auflösung als das cLSM. Das kommt dadurch zustande, dass die Rückapertur im ApoTome homogen ausgeleuchtet wird, während sie im cLSM die (abgeschnittene) Gausssche Intensitätsverteilung des Anregungslasers aufweist. Die axiale Auflösung ist im cLSM mit einer Ausnahme besser als im ApoTome: Dieses übertrifft das cLSM bei der Auflösung niedriger Raumfrequenzen unter Verwendung von Aufnahmeparametern, die auf optimale Auflösung abzielen (cLSM, 
$d_{\mathrm{p}}=0.4$ A.U. sowie ApoTome, „VL“-Gitter).

Im cLSM zeigt die axiale Auflösung eine zentral-symmetrische Verteilung über das Bildfeld, welche hauptsächlich durch Aberrationen entstehen, die mit dem Rasterverfahren zusammen hängen. Demgegenüber ist die axiale Auflösung des ApoTomes homogen innerhalb des Bildfeldes.

Die Mikroskopiemethode der strukturierten Beleuchtung mit einem linearen Gitter weist anisotrope Auflösung auf: Lineare Strukturen unterhalb der Auflösungsgrenze werden schlechter aufgelöst, wenn sie die gleiche Orientierung wie das Gitter aufweisen. Im Falle ähnlicher Raumfrequenzen wie das Gitter werden sie kontrastreicher dargestellt.

Bezogen auf die optische Schnittbildung ist die untersuchte Form der strukturierten Beleuchtung eingeschränkt auf dünne Präparate, da das PoIsson-Rauschen aus den Außerfokusebenen nicht herausgerechnet werden kann. Weiterhin muss das Präparat wenigstens für einige Sekunden stabil sein, da die Aufnahme der Rohdaten für ein einzelnes optisch geschnittenes Bild etwa eine Sekunde dauert. 


\section{Bibliography}

E. Abbe (1873). Beiträge zur Theorie des Mikroskops und der mikroskopischen Wahrnehmung. Schultzes Arc f Mikr Anat 9:413-468.

V. Andresen, A. Egner and S. W. Hell (2001). Time-multiplexed multifocal multiphoton microscope. Opt Lett 26(2):75-77. DOI: 10.1364/OL . 26.000075.

R. Arimoto and J. M. Murray (2004). A common aberration with water-immersion objective lenses. J Microsc 216(1):49-51. DOI: 10.1111/j.0022-2720.2004. 01383.x.

B. H. Armstrong (1967). Spectrum line profiles: the Voigt function. J Quant Spectrosc 7(1):61-88. DOI: 10.1016/0022-4073(67)90057-X.

K. C. Benny Lee, J. Siegel, S. E. Webb, S. Lévêque-Fort, M. J. Cole, R. Jones, K. Dowling, M. J. Lever and P. M. French (2001). Application of the stretched exponential function to fluorescence lifetime imaging. Biophys J 81(3):1265-1274. DOI: 10 . 1016/S0006-3495(01)75784-0.

J. Bewersdorf, R. Schmidt and S. W. Hell (2006). Comparison of I5M and 4Pimicroscopy. J Microsc 222(2): 105-117. DOI: 10.1111/j.1365-2818. 2006. 01578.x.

M. Born and E. Wolf (1999). Principles of Optics. 7th (expanded) ed. Cambridge: University Press.

A. Boyde (1985). Stereoscopic images in confocal (tandem scanning) microscopy. Science 230(4731):1270-1272. DOI: 10.1126/science.4071051.

K. Braeckmans, L. Peeters, N. N. Sanders, S. C. de Smedt and J. Demeester (2003). Three-dimensional fluorescence recovery after photobleaching with the confocal scanning laser microscope. Biophys $J$ 85(4):2240-2252. DOI: 10.1016/S00063495 (03) 74649-9.

G. J. Brakenhoff, G. W. H. Wurpel, K. Jalink, L. Oomen, L. Brocks and J. M. Zwier (2005). Characterization of sectioning fluorescence microscopy with thin uniform fluorescent layers: Sectioned Imaging Property or SIPcharts. J Microsc 219(3):122-132. DOI: 10.1111/j.1365-2818.2005.01504.x.

K. Carlsson, P. Danielsson, R. Lenz, A. Liljeborg, L. Majlöf and N. Åslund (1985). Three-dimensional microscopy using a confocal laser scanning microscope. Opt Lett 10(3):53-55. DOI: 10.1364/OL.10.000053.

F. Chasles, B. Dubertret and A. C. Boccara (2007). Optimization and characterization of a structured illumination microscope. Opt Express 15(24):16130-16140. DOI: $10.1364 /$ OE.15.016130. 
E. H. Cho and S. J. Lockett (2006). Calibration and standardization of the emission light path of confocal microscopes. J Microsc 223(1):15-25. DOI: $10.1111 / \mathrm{j}$. 1365-2818.2006.01598.x.

M. J. Cole, J. Siegel, S. E. D. Webb, R. Jones, K. Dowling, P. M. W. French, M. J. Lever, L. O. D. Sucharov, M. A. A. Neil, R. Juškaitis and T. Wilson (2000). Whole-field optically sectioned fluorescence lifetime imaging. Opt Lett 25(18):1361-1363. DOI: 10.1364/OL . 25 .001361.

I. J. Cox and C. J. R. Sheppard (1983). Scanning optical microscope incorporating a digital framestore and microcomputer. Appl Opt 22(10): 1474-1478. DOI: 10.1364/AO.22.001474.

W. Denk, J. H. Strickler and W. W. Webb (1990). Two-photon laser scanning fluorescence microscopy. Science 248(4951):73-76. DOI: 10 . 1126/science. 2321027.

R. R. Duncan, A. Bergmann, M. A. Cousin, D. K. Apps and M. J. Shipston (2004). Multi-dimensional time-correlated single photon counting (TCSPC) fluorescence lifetime imaging microscopy (FLIM) to detect FRET in cells. $J$ Microsc 215(1):1-12. DOI: 10.1111/j.0022-2720.2004.01343.x.

A. Egner, V. Andresen and S. W. Hell (2002). Comparison of the axial resolution of practical Nipkow-disk confocal fluorescence microscopy with that of multifocal multiphoton microscopy: theory and experiment. J Microsc 206(1):24-32. DOI: 10.1046/j.1365-2818.2002.01001.x.

A. Egner, C. Geisler, C. von Middendorff, H. Bock, D. Wenzel, R. Medda, M. Andresen, A.-C. Stiel, S. Jakobs, C. Eggeling, A. Schönle and S. W. Hell (2007). Fluorescence nanoscopy in whole cells by asynchronous localization of photoswitching emitters. Biophys J 93(9):3285-3290. DOI: 10.1529/biophysj. 107.112201.

T. Förster (1948). Zwischenmolekulare Energiewanderung und Fluoreszenz. Annalen der Physik 437(1):55-75. DOI: 10.1002/andp.19484370105.

M. Fulwyler, Q. S. Hanley, C. Schnetter, I. T. Young, E. A. Jares-Erijman, D. J. Arndt-Jovin and T. M. Jovin (2005). Selective photoreactions in a programmable array microscope (PAM): photoinitiated polymerization, photodecaging, and photochromic conversion. Cytometry A 67(2):68-75. DOI: 10.1002/cyto.a. 20174.

A. Gennerich and D. Schild (2000). Fluorescence correlation spectroscopy in small cytosolic compartments depends critically on the diffusion model used. Biophys J 79(6):3294-3306. DOI: 10.1016/S0006-3495(00)76561-1. 
A. Gennerich and D. Schild (2002). Anisotropic diffusion in mitral cell dendrites revealed by fluorescence correlation spectroscopy. Biophys $J$ 83(1):510-522. DOI: 10 . 1016/S0006-3495(02)75187-4.

E. Gratton, S. Breusegem, J. Sutin, Q. Ruan and N. Barry (2003). Fluorescence lifetime imaging for the two-photon microscope: time-domain and frequencydomain methods. J Biomed Opt 8(3):381-390. DOI: 10.1117/1.1586704.

M. G. L. Gustafsson (2000). Surpassing the lateral resolution limit by a factor of two using structured illumination microscopy. J Microsc 198(2):82-87. DOI: $10.1046 / j .1365-2818.2000 .00710 . x$.

M. G. L. Gustafsson, D. A. Agard and J. W. Sedat (1999). I5M: 3D widefield light microscopy with better than $100 \mathrm{~nm}$ axial resolution. J Microsc 195(1):10-16. DOI: $10.1046 / j .1365-2818.1999 .00576 . x$.

Q. S. Hanley, P. J. Verveer, M. J. Gemkow, D. Arndt-Jovin and T. M. Jovin (1999). An optical sectioning programmable array microscope implemented with a digital micromirror device. J Microsc 196(3):317-331. DOI: 10.1046/j.13652818.1999.00602.x.

Q. S. Hanley, P. J. Verveer, D. J. Arndt-Jovin and T. M. Jovin (2000). Threedimensional spectral imaging by hadamard transform spectroscopy in a programmable array microscope. J Microsc 197(1):5-14. DOI: 10.1046/j.1365$2818.2000 .00665 . x$.

Q. S. Hanley, K. A. Lidke, R. Heintzmann, D. J. Arndt-Jovin and T. M. Jovin (2005). Fluorescence lifetime imaging in an optically sectioning programmable array microscope (PAM). Cytometry A 67(2):112-118. DOI: 10.1002/cyto.a. 20177.

E. Hecht and A. Zajac (2003). Optics. 4th ed. San Francisco: Addison Wesley.

R. Heintzmann, Q. S. Hanley, D. Arndt-Jovin and T. M. Jovin (2001). A dual path programmable array microscope (PAM): simultaneous acquisition of conjugate and non-conjugate images. J Microsc 204(2):119-135. DOI: 10.1046/j.13652818.2001.00945.x.

R. Heintzmann (2006). Handbook of Biological Confocal Microscopy. Ed. by J. B. Pawley. 3rd ed. New York: Springer. Chap. 13. Structured Illumination Methods, pp. 265-279.

S. W. Hell (1992). Double-confocal microscope. Pat. 0491289.

S. W. Hell and M. Kroug (1995). Ground-state-depletion fluorescence microscopy: a concept for breaking the diffraction resolution limit. Appl Phys B 60(5):495-497. DOI: $10.1007 / \mathrm{BF} 01081333$.

S. W. Hell and E. H. K. Stelzer (1992). Properties of a 4Pi-confocal fluorescence microscope. J Opt Soc Am A 9(12):2159-2166. DOI: 10.1364/JOSAA.9.002159. 
S. W. Hell, G. Reiner, C. Cremer and E. H. K. Stelzer (1993). Aberrations in confocal fluorescence microscopy induced by mismatches in refractive index. $J$ Microsc 169(3):391-405.

S. W. Hell and J. Wichmann (1994). Breaking the diffraction resolution limit by stimulated emission depletion: stimulated-emission-depletion fluorescence microscopy. Opt Lett 19(11):780-782. DOI: 10.1364/OL . 19.000780.

B. J. Howell, F. W. Baumgardner, K. Bondi and H. Rahn (1970). Acid-base balance in cold-blooded vertebrates as a function of body temperature. Am J Physiol 218(2):600-606.

T. Ida, M. Ando and H. Toraya (2000). Extended pseudo-Voigt function for approximating the Voigt profile. J Appl Cryst 33(6):1311-1316. DOI: 10.1107/ S0021889800010219.

D. Karadaglić (2004). "Wide-field optical sectioning microscopy". PhD thesis. University of Oxford.

D. Karadaglić and T. Wilson (2008). Image formation in structured illumination wide-field fluorescence microscopy. Micron 39(7):808-818. DOI: 10.1016/ j . micron.2008.01.017.

S. A. Kim, K. G. Heinze and P. Schwille (2007). Fluorescence correlation spectroscopy in living cells. Nat Methods 4(11):963-973. DOI: 10.1038/nmeth1104.

L. C. Kuypers, J. J. J. Dirckx and W. F. Decraemer (2004). A simple method for checking the illumination profile in a laser scanning microscope and the dependence of resolution on this profile. Scanning 26(5):256-258. DOI: 10.1002/ sca. 4950260507.

B. C. Lagerholm, S. Vanni, D. L. Taylor and F. Lanni (2003). Cytomechanics applications of optical sectioning microscopy. Methods Enzymol 361:175-197. DOI: $10.1016 /$ S0076-6879(03)61011-6.

J. R. Lakowicz (2006). Principles of Fluorescence Spectroscopy. 3rd ed. Springer. Chap. 4.9 Data analysis: nonlinear least squares, pp. 129-154.

R. Lansford, G. Bearman and S. E. Fraser (2001). Resolution of multiple green fluorescent protein color variants and dyes using two-photon microscopy and imaging spectroscopy. J Biomed Opt 6(3):311-318. DOI: 10.1117/1.1383780.

D. S. Lidke and D. J. Arndt-Jovin (2004). Imaging takes a quantum leap. Physiology (Bethesda) 19:322-325. DOI: 10.1152/physiol.00030.2004.

K. A. Lidke, B. Rieger, D. S. Lidke and T. M. Jovin (2005). The role of photon statistics in fluorescence anisotropy imaging. IEEE Trans Image Process 14(9): 1237-1245. DOI: 10.1109/TIP. 2005.852458. 
E. H. Linfoot and E. Wolf (1956). Phase distribution near focus in an aberrationfree diffraction image. Proc Phys Soc B 69(8):823-832. DOI: 10.1088/03701301/69/8/307.

W. Lukosz and M. Marchand (1963). Optischen Abbildung unter Überschreitung der beugungsbedingten Auflösungsgrenze. Opt Acta 10:241-255. DOI: 10.1080/ 713817795 .

M. Minsky (1961). Microscopy apparatus. Pat. 3013467. Dec. 1961.

J. Mitić, T. Anhut, M. Meier, M. Ducros, A. Serov and T. Lasser (2003). Optical sectioning in wide-field microscopy obtained by dynamic structured light illumination and detection based on a smart pixel detector array. Opt Lett 28(9): 698-700. DOI: 10.1364/OL.28.000698.

M. A. Model and J. L. Blank (2008). Concentrated dyes as a source of twodimensional fluorescent field for characterization of a confocal microscope. $J$ Microsc 229(1):12-16. DOI: 10.1111/j.1365-2818.2007.01880.x.

S. Monneret, M. Rauzi and P.-F. Lenne (2006). Highly flexible whole-field sectioning microscope with liquid-crystal light modulator. J Opt A: Pure Appl Opt 8: S461-S466. DOI: 10.1088/1464-4258/8/7/S23.

J. M. Murray, P. L. Appleton, J. R. Swedlow and J. C. Waters (2007). Evaluating performance in three-dimensional fluorescence microscopy. J Microsc 228(3): 390-405. DOI: $10.1111 / \mathrm{j} .1365-2818.2007 .01861 . \mathrm{x}$.

H. Naora (1955). Microspectrophotometry of cell nucleus stained by feulgen reaction. I. Microspectrophotometric apparatus without Schwarzschild-Villiger effect. Exp Cell Res 8(2):259-278. DOI: 10.1016/0014-4827(55)90141-5.

M. A. A. Neil, R. Juškaitis and T. Wilson (1997). Method of obtaining optical sectioning by using structured light in a conventional microscope. Opt Lett 22(24):1905-1907. DOI: 10.1364/OL . 22.001905.

M. A. A. Neil, A. Squire, R. Juškaitis, P. I. Bastiaens and T. Wilson (2000). Wide-field optically sectioning fluorescence microscopy with laser illumination. J Microsc 197(1):1-4. DOI: 10.1046/j.1365-2818.2000.00656.x.

M. A. A. Neil, R. Juškaitis and T. Wilson (1998). Real time 3D fluorescence microscopy by two beam interference illumination. Opt Comm 153(1-3):1-4. DOI: 10 .1016/S0030-4018(98)00210-7.

H. Niedrig, ed. (2004). Optik. 10. Vol. 3. Bergmann - Schaefer - Lehrbuch der Experimentalphysik. Berlin, New York: Walter de Gruyter.

R. Niesner, V. Andresen, J. Neumann, H. Spiecker and M. Gunzer (2007). The power of single and multibeam two-photon microscopy for high-resolution and high-speed deep tissue and intravital imaging. Biophys J 93(7):2519-2529. DOI: 10.1529/biophys j . 106 . 102459 . 
P. D. Nieuwkoop and J. Faber, eds. (1994). Normal Table of Xenopus laevis (Daudin). Garland Publishing.

H. Nyquist (1924). Certain factors affecting telegraph speed. Bell System Technical Journal 3:324-346.

H. Nyquist (1928). Certain topics in telepgraph transmission theory. A $I E E$ Transactions 47:617-644. DOI: 10.1109/5.989875.

P. Pankajakshan, B. Zhang, L. Blanc-Feraud, Z. Kam, J.-C. Olivo-Marin and J. Zerubia (2007). Parametric blind deconvolution for confocal laser scanning microscopy. Conf Proc IEEE Eng Med Biol Soc 2007:6532-6535. DOI: 10.1109/ IEMBS . 2007.4353856.

J. B. Pawley, ed. (2006). Handbook of Biological Confocal Microscopy. 3rd rev. ed. Berlin: Springer.

M. Petráň, M. Hadravský, D. M. Egger and R. Galambos (1968). Tandem-scanning reflected-light microscope. J Opt Soc Am 58(5):661-664. DOI: 10.1364/JOSA . 58.000661.

H. Qian and E. L. Elson (1991). Analysis of confocal laser-microscope optics for 3D fluorescence correlation spectroscopy. Appl Opt 30(10):1185-1195. DOI: 10.1364/A0.30.001185.

J. Reisert and J. Bradley (2005). Activation of olfactory cyclic-nucleotide gated channels revisited. J Physiol 569(1):4-5. DOI: 10.1113/jphysiol.2005.09660 2.

D. R. Sandison, D. W. Piston, R. M. Williams and W. W. Webb (1995). Quantitative comparison of background rejection, signal-to-noise ratio, and resolution in confocal and full-field laser scanning microscopes. Appl Opt 34(19):3576-3588. DOI: $10.1364 / \mathrm{AO} .34 .003576$.

W. G. J. H. M. van Sark, P. L. T. M. Frederix, A. A. Bol, H. C. Gerritsen and A. Meijerink (2002). Blueing, bleaching and blinking of single CdSe/ZnS quantum dots. Chem Phys Chem 3(10):871-879. DOI: 10 .1002/1439-7641 (20021018)3: 10<871: :AID-CPHC871>3.0.CO;2-T.

L. H. Schaefer, D. Schuster and H. Herz (2001). Generalized approach for accelerated maximum likelihood based image restoration applied to three-dimensional fluorescence microscopy. J Microsc 204(2): 99-107. DOI: 10.1046/j .13652818.2001.00949.x.

L. H. Schaefer, D. Schuster and J. Schaffer (2004). Structured illumination microscopy: artefact analysis and reduction utilizing a parameter optimization approach. J Microsc 216(2):165-174. DOI: 10.1111/j.0022-2720.2004.01411.x.

L. Schermelleh, P. M. Carlton, S. Haase, L. Shao, L. Winoto, P. Kner, B. Burke, M. C. Cardoso, D. A. Agard, M. G. L. Gustafsson, H. Leonhardt and J. W. 
Sedat (2008). Subdiffraction multicolor imaging of the nuclear periphery with 3D structured illumination microscopy. Science 320(5881): 1332-1336. DOI: 10.1126/science. 1156947 .

D. Schild (1996). Laser scanning microscopy and calcium imaging. Cell Calcium 19(4):281-296. DOI: 10 .1016/S0143-4160 (96) 90069-X.

M. Schrader, M. Hofmann and S. W. Hell (1998). Ultrathin fluorescent layers for monitoring the axial resolution in confocal and two-photon fluorescence microscopy. J Microsc 191(2): 135-140. DOI: 10 .1046/ j .1365-2818 . 1998 . $00361 . \mathrm{x}$.

M. Schwertner, M. J. Booth and T. Wilson (2005). Simple optimization procedure for objective lens correction collar setting. J Microsc 217(3):184-187. DOI: $10.1111 / j .1365-2818.2005 .01431 . x$.

C. E. Shannon (1949). Communication in the presence of noise. Proc IEEE 37(1): $10-21$.

L. Shao, B. Isaac, S. Uzawa, D. A. Agard, J. W. Sedat and M. G. L. Gustafsson (2008). I5S: widefield light microscopy with 100-nm-scale resolution in three dimensions. Biophys J 94(12):4971-4983. DOI: 10.1529/biophysj.107.12035 2.

C. J. R. Sheppard and A. Choudhury (1977). Image formation in the scanning microscope. Opt Acta 24(10):1051-1073. DOI: 10.1080/713819421.

C. J. R. Sheppard and P. Török (1997). Effects of specimen refractive index on confocal imaging. J Microsc 185:366-374. DOI: 10.1046/j .1365-2818. 1997. $\mathrm{d} 01-627 . \mathrm{x}$.

O. Shimomura (2005). The discovery of aequorin and green fluorescent protein. $J$ Microsc 217(1):1-15. DOI: 10.1111/j.0022-2720.2005.01441.x.

O. Shimomura, F. H. Johnson and S. Y. (1962). Extraction, purification and properties of aequorin, a bioluminescent protein from the luminous hydromedusan, Aequorea. J Cell Comp Physiol 59:223-239. DOI: 10.1002/jcp. 1030590302.

J. Siegel, D. S. Elson, S. E. D. Webb, K. C. B. Lee, A. Vlandas, G. L. Gambaruto, S. Lévêque-Fort, M. J. Lever, P. J. Tadrous, G. W. H. Stamp, A. L. Wallace, A. Sandison, T. F. Watson, F. Alvarez and P. M. W. French (2003). Studying biological tissue with fluorescence lifetime imaging: microscopy, endoscopy, and complex decay profiles. Appl Opt 42(16): 2995-3004. DOI: 10 .1364/A0 . 42 . 002995.

P. A. Stokseth (1969). Properties of a defocused optical system. J Opt Soc Am 59(10):1314-1321. DOI: 10.1364/JOSA . 59.001314.

F. A. Tanious, J. M. Veal, H. Buczak, L. S. Ratmeyer and W. D. Wilson (1992). DAPI (4',6-diamidino-2-phenylindole) binds differently to DNA and RNA: 
minor-groove binding at AT sites and intercalation at AU sites. Biochemistry 31(12):3103-3112. DOI: 10.1021/bi00127a010.

P. Thompson, D. E. Cox and J. B. Hastings (1987). Rietveld refinement of DebyeScherrer synchrotron x-ray data from $\mathrm{Al}_{2} \mathrm{O}_{3}$. J. Appl. Cryst. 20(2):79-83. DOI: 10.1107/S0021889887087090.

R. Y. Tsien (1988). Fluorescence measurement and photochemical manipulation of cytosolic free calcium. Trends Neurosci 11(10):419-424. DOI: 10 .1016/01662236 (88)90192-0.

R. Y. Tsien (1989a). Fluorescent indicators of ion concentrations. Methods Cell Biol 30:127-156. DOI: 10.1016/S0091-679X (08)60978-4.

R. Y. Tsien (1989b). Fluorescent probes of cell signaling. Annu Rev Neurosci 12: 227-253. DOI: 10.1146/annurev.ne.12.030189.001303.

H. Tsurui, H. Nishimura, S. Hattori, S. Hirose, K. Okumura and T. Shirai (2000). Seven-color fluorescence imaging of tissue samples based on Fourier spectroscopy and singular value decomposition. J Histochem Cytochem 48(5):653-662.

G. Vicidomini, M. Schneider, P. Bianchini, S. Krol, T. Szellas and A. Diaspro (2007). Characterization of uniform ultrathin layer for z-response measurements in three-dimensional section fluorescence microscopy. J Microsc 225(1):88-95. DOI: $10.1111 / j .1365-2818.2007 .01718 . x$.

W. Voigt (1912). Über das Gesetz der Intensitätsverteilung innerhalb der Linien eines Gasspektrums. Münch Ber, 603.

H. T. M. van der Voort and G. J. Brakenhoff (1990). 3-D image formation in high-aperture fluorescence confocal microscopy: a numerical analysis. J Microsc 158(1):43-54.

E. Wang, C. M. Babbey and K. W. Dunn (2005). Performance comparison between the high-speed Yokogawa spinning disc confocal system and single-point scanning confocal systems. J Microsc 218(2):148-159. DOI: 10.1111/j.13652818.2005.01473.x.

G. K. Wertheim, M. A. Butler, K. W. West and D. N. E. Buchanan (1974). Determination of the Gaussian and Lorentzian content of experimental line shapes. Rev. Sci. Instrum. 45(11):1369-1371. DOI: 10.1063/1.1686503.

V. Westphal, S. O'Rizzoli, M. A. Lauterbach, D. Kamin, R. Jahn and S. W. Hell (2008). Video-rate far-field optical nanoscopy dissects synaptic vesicle movement. Science 320(5873):246-249. DOI: 10.1126/science.1154228.

T. Wilson, ed. (1990). Confocal Microscopy. London, San Diego: Academic Press.

J. Wlodarczyk, A. Woehler, F. Kobe, E. Ponimaskin, A. Zeug and E. Neher (2008). Analysis of FRET signals in the presence of free donors and acceptors. Biophys J 94(3):986-1000. DOI: 10.1529/biophysj.107.111773. 
R. Wolleschensky, B. Zimmermann and M. Kempe (2006). High-speed confocal fluorescence imaging with a novel line scanning microscope. J Biomed Opt 11(6):064011. DOI: 10.1117/1.2402110.

F. S. Wouters, P. I. Bastiaens, K. W. Wirtz and T. M. Jovin (1998). FRET microscopy demonstrates molecular association of non-specific lipid transfer protein (nsL-TP) with fatty acid oxidation enzymes in peroxisomes. EMBO J 17(24):7179-7189. DOI: 10.1093/emboj/17.24.7179.

R. M. Zucker and J. M. Lerner (2005). Wavelength and alignment tests for confocal spectral imaging systems. Microsc Res Tech 68(5):307-319. DOI: $10.1002 /$ jemt. 20249.

R. M. Zucker, P. Rigby, I. Clements, W. Salmon and M. Chua (2007). Reliability of confocal microscopy spectral imaging systems: use of multispectral beads. Cytometry A 71(3):174-189. DOI: 10.1002/cyto.a.20371.

J. M. Zwier, G. J. van Rooij, J. W. Hofstraat and G. J. Brakenhoff (2004). Image calibration in fluorescence microscopy. J Microsc 216(1):15-24. DOI: $10.1111 / j .0022-2720.2004 .01390 . x$.

J. M. Zwier, L. Oomen, L. Brocks, K. Jalink and G. J. Brakenhoff (2008). Quantitative image correction and calibration for confocal fluorescence microscopy using thin reference layers and SIPchart-based calibration procedures. J Microsc 231(1):59-69. DOI: 10.1111/j.1365-2818.2008.02017.x. 


\section{Abbreviations}

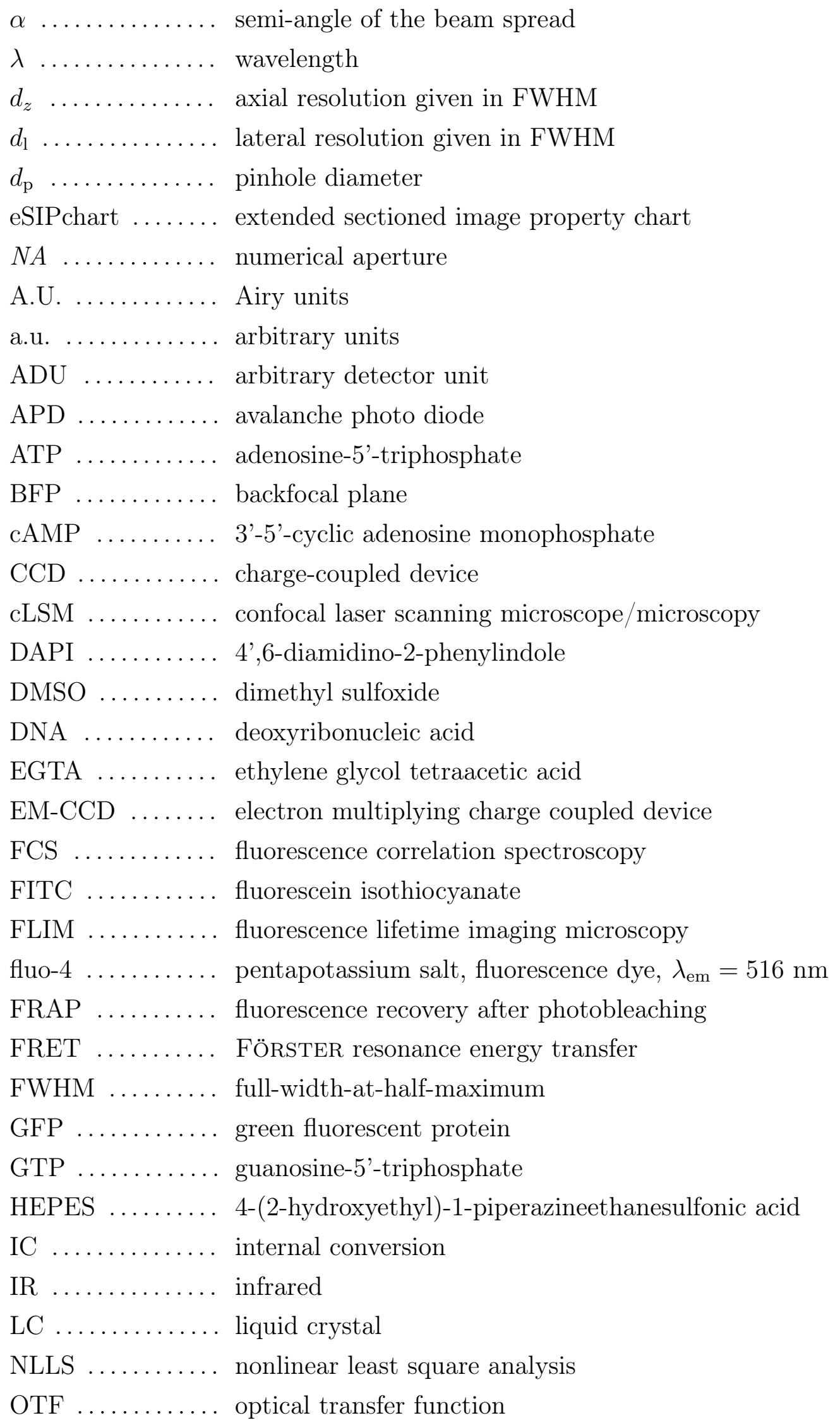


PALMIRA $\ldots . .$. photoactivation localization microscopy with independent running acquisition

PBS $\ldots \ldots \ldots \ldots$ phosphate buffered saline

$\mathrm{pH} \ldots \ldots \ldots \ldots$ cologarithm of the activity of dissolved hydrogen ions

PMT $\ldots \ldots \ldots \ldots$ photomultiplier tube

PSF $\ldots \ldots \ldots \ldots$ point spread function

$\mathrm{S} / \mathrm{B} \ldots \ldots \ldots$ signal-to-background-ratio

SIM $\ldots . . . \ldots . .$. structured illumination microscopy

SNR $\ldots \ldots \ldots \ldots$ signal-to-noise-ratio

SPA .......... single photon absorption

STED .......... stimulated emission depletion

TPA $\ldots . . \ldots$. two photon absorption

UV $\ldots \ldots \ldots \ldots$ ultraviolet

$\mathrm{VH} \ldots \ldots \ldots \ldots$ ApoTome grid for $h$ igh $N A$ objectives; $17.5 \frac{\text { lines }}{\mathrm{mm}}$

VL $\ldots \ldots \ldots \ldots$ ApoTome grid for low $N A$ objectives; $35 \frac{\text { lines }}{\mathrm{mm}}$ 


\section{Curriculum Vitae}

\begin{tabular}{ll} 
PERSONAL DATA & \\
\hline Name & Arwed Weigel \\
Date of birth & 16.07 .1979 \\
Place of birth & Kassel \\
Nationality & German \\
Current address & Barfüßerstraße 16 \\
& 37073 Göttingen \\
& Germany \\
Phone & +495513912201 \\
& +4917623517669 \\
e-mail & aweigel1@gwdg.de
\end{tabular}

EDUCATION

since $07 / 2005$

PhD in the laboratory of Prof. Dr. Dr. Schild

Department of Neurophysiology and Cellular

Biophysics, University of Göttingen, Germany

Advisor: Dr. André Zeug

$06 / 2005$

M.Sc. Neurosciences, graduate program of the International Max Planck Research School 'Neurosciences', Göttingen, Germany

Thesis: Resolution in the ApoTome and the cLSM: a Comparison.

Department of Neurophysiology and Cellular Biophysics, University of Göttingen, Germany

Advisors: Prof. Dr. Dr. Detlev Schild and Prof. Dr. Walther Stühmer

since $09 / 2003$ International Max Planck Research School 'Neurosciences', Göttingen, Germany

$10 / 2002$ Vordiplom in physics, University of Potsdam, Germany

10/2000 - 09/2003 Undergraduate studies in physics, University of Potsdam, Germany

07/1999 - 06/2000 Military service, Fritzlar, Germany

$06 / 1999$

Abitur, Friedrichsgymnasium Kassel, Germany 


\section{List of Publications}

A. Weigel, D. Schild, and A. Zeug (2009). Resolution in the ApoTome and the cLSM: a comparison.

J. Biomed. Opt. 14(1):014022.

P. Balcarek, A. Kuhn, A. Weigel, T. A. Walde, M. Wachowski, K. M. Stürmer, and K. H. Frosch. Impact of monopolar radiofrequency energy on subchondral bone viability - an in vivo study.

Under review

A. Weigel and A. Zeug. eSIPcharts for the calibration and quantitation in optically sectioning fluorescence microscopy.

In preparation 